\title{
Spacetime coverings and the casual boundary
}

\author{
Luis Alberto Aké ${ }^{a}$ and Jónatan Herrera ${ }^{b}$ \\ ${ }^{a}$ Departamento de Álgebra, Geometría y Topología, Facultad de Ciencias, Universidad de Málaga, \\ Campus Teatinos, Málaga, Spain \\ ${ }^{\mathrm{b}}$ Departamento de Matemática, Universidade Federal de Santa Catarina, \\ Campus Universitario de Trindade, Florianopolis, Brazil \\ E-mail: luisake@uma.es, jonatanhf@gmail.es
}

ABSTRACT: We consider the relation between the c-completion of a Lorentz manifold $\mathrm{V}$ and its quotient $M=V / G$, where $G$ is an isometry group acting freely and properly discontinuously. First, we consider the future causal completion case, characterizing virtually when such a quotient is well behaved with the future chronological topology and improving the existing results on the literature. Secondly, we show that under some general assumptions, there exists a homeomorphism and chronological isomorphism between both, the c-completion of $M$ and some adequate quotient of the c-completion of $V$ defined by $G$. Our results are optimal, as we show in several examples. Finally, we give a practical application by considering isometric actions over Robertson-Walker spacetimes, including in particular the Anti-de Sitter model.

Keywords: Classical Theories of Gravity, Differential and Algebraic Geometry, Discrete Symmetries, AdS-CFT Correspondence

ARXIV EPRINT: 1605.03128 


\section{Contents}

1 Introduction 1

2 Preliminaries 5

2.1 Sequential topologies and limit operators 5

2.2 C-boundary construction 9

2.3 Spacetime covering projections: the causal ladder and main properties 12

3 Partial Boundaries under the action of the group 13

$\begin{array}{ll}3.1 \text { Proof of theorem } 1.1 & 19\end{array}$

4 The C-completion under the action of the group 19

$\begin{array}{lll}4.1 & \text { Point set level } & 19\end{array}$

4.2 At the chronological level 23

4.3 At the topological level 24

$\begin{array}{ll}4.4 & \text { Group actions with the finite chronology property }\end{array}$

4.5 Proof of theorem $1.2 \quad 31$

5 On the optimality of the results: some examples 31

6 A physical application: quotients on Robertson-Walker spacetimes 42

\section{Introduction}

The AdS/CFT correspondence, also known as Maldacena's duality, states the duality between gravitational theories, as string or M-theory, on a bulk space (usually a product of the Anti-de Sitter spacetime with spheres or other compact sets) and conformal field theories defined on the boundary of the bulk space which behaves as a hologram of inferior dimension (see [1]). As it is apparent, the conjecture relies strongly in the notion of boundary of Lorentz manifolds. However, the problem to attach a natural boundary for any Lorentz manifold encoding relevant information on it, as its conformal structure and related elements (event horizons, singularities, etc.) has been a long standing issue along the last four decades.

Among the several constructions proposed (see [2-4] for nice reviews on the classical elements and $[5,6]$ for updated progress), two approaches have had a specially important role in general relativity, the conformal and the causal boundaries.

The conformal boundary is the most applied one in mathematical relativity and several notions, as asymptotic flatness or tools as Penrose-Carter diagrams rely on it. Even in the original approach of the AdS/CFT correspondence, it is the conformal boundary the 
chosen as the holographic one. In fact, the Anti-de Sitter spacetime can be conformally embedded in the Lorentz-Minkowski model, obtaining a simple (and non-compact) conformal boundary. However, it has important limitations as it is an ad hoc construction: no general formalism determines when the boundary of a reasonably general spacetime is definable, intrinsic, unique and contain useful information of the spacetime (see [7] and [5, section 4] for studies regarding the uniqueness of the conformal boundary). In fact, as it was putted forward by Bernstein, Maldacena and Nastase [8]), there seems to be problems when the conformal boundary is considered on plane waves. Indeed, Marolf and Ross [9] realized that the conformal boundary is not available for non-conformally flat plane waves. So, they proposed a redefinition of the c-boundary applicable to such waves [10] which was refined and systematically studied by Flores and Sánchez in [11].

This motivated a reconsideration of such constructions by substituting the conformal boundary by the causal one, which is intrinsic, conformally invariant and it can be computed systematically, as it was carried out in [5]. It is worth emphasizing that both the conformal and causal boundaries are shown to coincide in most relevant classes (so, previous results based on the conformal case are not required to be re-obtained for the causal one).

Returning to the problem of AdS/CFT correspondence, it is our aim to present the causal boundary of different classes of Lorentz manifolds, allowing the study of such a correspondence with different bulk spaces. In this sense let $M$ be, for instance, a Lorentz manifold with constant negative curvature, and so, a spacetime that can be locally modelled by the Anti-de Sitter spacetime. Recalling that the universal covering Ad̃ $\mathrm{d}$ is maximal, simply-connected and with constant negative curvature, it is expectable that $M$ can be described as a quotient space of $A \tilde{d} S$ by an appropriate group of isometries (in fact, for certain spacetime topologies, the existence of such an appropriate group was proved by Mess [12]). This is the particular case of the BTZ blackholes, the $(2+1)$-model of spacetime first introduced by Bañados, Teitelboim and Zanelli [13]; and the Hawking-Page reference space [14], whose representations as a quotient of the Anti-de Sitter model are well known [15? , 16].

Due the fact that the causal boundary is well known for ÃdS (see [17, section 4.1]), the following question, particularly natural from the mathematical viewpoint, arises: given two (general) Lorentz manifolds $\mathrm{M}$ and $\mathrm{V}$ where $\mathrm{M}$ is constructed as the quotient of $\mathrm{V}$ by some group of isometries, what is the relation between the causal boundaries and completions of $M$ and $V$ ? An adequate answer for this question will give us tools to easily compute the causal completion of $M$ once we know the corresponding on $V$. For instance, such a result will be applicable to models like the BTZ blackholes or the Hawking-Page reference model, besides other models constructed in a similar way (as the case of Cosmic Strings, see [18]). It will also give us relevant information of the c-completion on $\mathrm{V}$ whenever the c-completion in $M$ is known.

The first studies in this direction are due to Harris [19]. In his work, he studied how isometrical actions affect the causal structures of the spacetimes, with special attention to the future causal boundary and related concepts (as strong causality). Concretely, he considers a projection $\pi: \mathrm{V} \rightarrow \mathrm{M}$ given by a discrete subgroup $\mathrm{G}$ of isometries acting freely and properly discontinuously in $V$, i.e., where $M=V / G$ and the elements on $M$ 
represents G-orbits in V. In this settings, Harris characterizes the strong causality and global hyperbolicity of $M$ in terms of the global causal structure of $V$. Moreover, and under the assumption of $M$ being distinguishing (which implies, in particular, that $V$ also is), he presents necessary conditions in order to ensure when the future causal completion of $\mathrm{M}$ is homeomorphic to an appropiatre quotient of the future causal completion of $\mathrm{V}$.

Our aim in this work is to extend the results obtained by Harris for the future causal completion to the c-completion. However, several problems have to be addressed first. On the one hand, the main result in [19] imposes that both, the future causal boundary of $M$ and $\mathrm{V}$ have only spacelike future boundaries. This condition, even if reasonable (specially recalling the final example of his paper), is too strong for the c-completion context, where particularly timelike boundary points are specially relevant. On the other hand, and in spite with the partial case, the c-completion requires the study of the so-called S-relation between future and past sets, as well as some "compatibility" between the topology of the future and past completions.

The contents of the paper are organized as follow. In section 2 we will give the preliminaries that we are going to need along the rest of the paper. Most of them are well known (for instance, the construction of the c-completion was developed in [5]), but we have also introduced concepts (as first order UTS, definition 2.1) and results (lemmas 2.2 and 2.4; and some of the assertions in Theorem 2.10) that, as far as we know, are new.

Section 3 is devoted to the study of the future (and, by analogy, past) causal boundary. Here, at the point set level, we will recall the bijection $\hat{\jmath}$ defined by Harris between a suitable quotient of $\hat{V}$ (the future completion of $V$ ) and $\hat{M}$ (the future completion of $M$ ). Then, we will perform a detailed comparison between the topologies in both spaces (the first one with the induced quotient topology). The results of this section are summarized as follow:

Theorem 1.1. Let $\pi: \mathrm{V} \rightarrow \mathrm{M}$ be a spacetime covering projection (see section 2.3) and denote by $\hat{\pi}$ the extension to future c-completions (3.1). Let $\hat{\mathrm{V}} / \hat{\mathrm{G}}$ be the quotient space

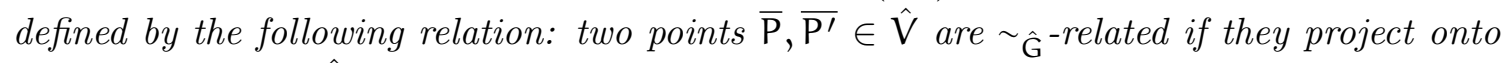
the same point in $\hat{M}$. Then, we obtain the following commutative diagram:

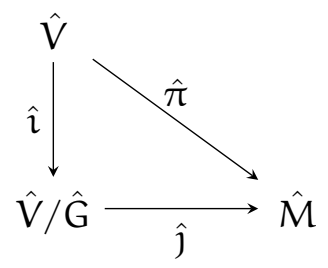

where $\hat{\imath}$ is the natural quotient projection. From construction, the map $\hat{\jmath}$ is bijective. At the topological level,

(i) The map $\hat{\jmath}$ is open.

(ii) If $\mathrm{M}$ does not admit sequences with future divergent lifts (definition 3.6), the map $\hat{\pi}$ (and so, $\hat{\jmath}$ ) is continuous. The converse also follows if we have that $\hat{\mathrm{L}}_{\mathrm{M}}$ is of first order UTS (definition 2.1). 
In particular, if $\mathrm{M}$ has only spatial future boundary points, $\hat{\jmath}$ is a homeomorphism between $\hat{\mathrm{V}} / \hat{\mathrm{G}}$ and $\hat{\mathrm{M}}$. The same result follows if $\mathrm{G}$ is finite and $\hat{\mathrm{V}}$ is Hausdorff.

As we can see on previous (ii), we have obtained almost a characterization of the continuity of $\hat{\jmath}$, up to the first order UTS property. In fact, such a result generalizes [19, theorem 3.4], as the last assertion of theorem 1.1 shows.

Section 4 is focused on the study of the (total) c-completion at all possible levels, namely at the point set, at the chronological and at the topological level. In section 4.1 are given simple and general sufficient conditions to ensure the definition of the map $\bar{\jmath}$ between a reasonable quotient of $\bar{V}$ (the c-completion of $\mathrm{V}$ ) and $\bar{M}$ (the c-completion on $M$ ). Then, it is shown in section 4.2 that previous map is well behaved respect to the chronological relation, whenever an appropriate chronological relation is defined on the quotient space. Finally, in sections 4.3 and 4.4, it is studied the conditions to ensure that the map $\bar{\jmath}$ is both, continuous and open respectively. Now the latter becomes subtler and a simple condition (to be finitely chronological) is introduced. This property also simplifies the conditions to ensure the well posedness and continuity of $\bar{\jmath}$.

Concretely, the results of such a section are summarized in the following:

Theorem 1.2. Let $\pi: \mathrm{V} \rightarrow \mathrm{M}$ be a spacetime covering projection and consider $\bar{\pi}: \overline{\mathrm{V}} \rightarrow$ $\hat{M}_{\emptyset} \times \check{M}_{\emptyset}$ one extension map as defined on definition 4.4 (see also remark 4.5). Then:

(PS1) If $M$ does not admit an inextensible sequence $\left\{x_{n}\right\}_{n} \subset M$ which is either past-directed chronological with future divergent lifts nor future-directed chronological with past divergent lifts; and any $(\mathrm{P}, \mathrm{F}) \in \overline{\mathrm{M}}$ with $\mathrm{P} \neq \emptyset \neq \mathrm{F}$ admits a lift on $\overline{\mathrm{V}}$ (in particular if $(\mathrm{V}, \mathrm{G})$ is finitely chronological, see definition 4.14), then the projection restricts properly to $\bar{M}$ and it is surjective.

(PS2) If, in addition, the projection $\pi$ is tame (recall definition 3.2) or $(\mathrm{V}, \mathrm{G})$ is finite chronological (see definition 4.14), $\bar{\pi}$ is univocally determined (see remark 4.5) and just reads as

$$
\bar{\pi}((\bar{P}, \bar{F}))=(\hat{\pi}(\bar{P}), \check{\pi}(\bar{F}))
$$

where $\hat{\pi}(\emptyset)=\check{\pi}(\emptyset)=\emptyset$.

(PS3) Finally, if $(\mathrm{V}, \mathrm{G})$ is finitely chronological and both $\hat{\mathrm{V}}, \grave{\mathrm{V}}$ are Hausdorff then the projection $\bar{\pi}$ restricts properly to $\bar{M}$, it is surjective and univocally determined.

Moreover, when the map $\bar{\pi}$ restricts properly to $\bar{M}$ and it is surjective, it defines the following relation between points in $\overline{\mathrm{V}}$ : two points are $\sim_{\mathrm{G}}$-related if they project onto the same point in $\overline{\mathrm{M}}$. Then, denoting by $\overline{\mathrm{V}} / \mathrm{G}$ the quotient space, we obtain the following commutative diagram:

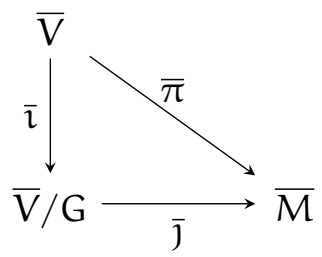

where $\bar{\imath}$ is the natural projection to the quotient and $\bar{\jmath}$ is the induced bijection. 
At the chronological level, and once an appropriate chronological relation is defined on $\overline{\mathrm{V}} / \mathrm{G}$ (see section 4.2), it follows that:

(CH) the map $\bar{\jmath}$ is a chronological isomorphism.

Finally, at the topological level, $\bar{\jmath}$ satisfies the following properties:

(TP1) The map $\bar{\jmath}$ is continuous if one of the following hypotheses hold:

(i) $\bar{\pi}$ satisfies that $\bar{\pi}((\overline{\mathrm{P}}, \emptyset))=(\mathrm{P}, \emptyset)$ and $\bar{\pi}((\emptyset, \overline{\mathrm{F}}))=(\emptyset, \mathrm{F})$ (this follows if, for instance, $\pi$ is tame or $(\mathrm{V}, \mathrm{G})$ is finite chronological); and $\mathrm{M}$ has no sequence with (future or past) divergent lifts.

(ii) $\bar{\pi}((\overline{\mathrm{P}}, \emptyset))=(\mathrm{P}, \emptyset), \bar{\pi}((\emptyset, \overline{\mathrm{F}}))=(\emptyset, \mathrm{F})$ and $\overline{\mathrm{M}}$ has no lightlike boundary points (see definition 2.5).

(TP2) If $(\mathrm{V}, \mathrm{G})$ is finite chronological, the map $\bar{\jmath}$ is open.

In particular, $\bar{\pi}$ restricts properly to $\overline{\mathrm{M}}$, it is surjective, univocally determined and induces a homeomorphism and chronological isomorphism between $\bar{V} / \mathrm{G}$ and $\bar{M}$ if it is satisfied one of the following assertions:

(a) $(\mathrm{V}, \mathrm{G})$ is finite chronological and $\mathrm{M}$ admits no sequence with (future or past) divergent lifts.

(b) $(\mathrm{V}, \mathrm{G})$ is finitely chronological, both $\hat{\mathrm{V}}, \check{\mathrm{V}}$ are Hausdorff and $\overline{\mathrm{M}}$ has no lightlike boundary points.

(c) $(\mathrm{V}, \mathrm{G})$ is finitely chronological, $\overline{\mathrm{V}}$ has no lightlike boundary points, and both $\hat{\mathrm{V}}, \grave{\mathrm{V}}$ are Hausdorff and have closed $\mathrm{G}$-orbits. In particular, if $\pi$ is (future and past) tame and there are no constant sequences with divergent lifts in $\mathrm{M}$, then the $\mathrm{G}$-orbits in $\hat{\mathrm{V}}$ and $\check{\mathrm{V}}$ will be closed.

In section 5 we include several technical examples showing the optimality of our results, that is, we show that if we remove any of our sufficient conditions (tameness, no existence of sequences with divergent lifts or finite chronology), the results are, in general, false. Finally in section 6, and as a physically relevant application of our result, we use the developed theory to compute the causal boundary of quotients of Robertson Walker spacetimes, including quotients of the AdS Spacetime.

\section{Preliminaries}

\subsection{Sequential topologies and limit operators}

Along this section we will include all the basic facts about sequential topologies and limit operators that we will require for the rest of the paper. Most of the results are known (see $[6,20]$ ), but we present the concept of first order UTS along some associated results that, as far as we known, are new. 
Let $X$ be an arbitrary space with a limit operator $L$ defined on it, that is, an operator $L: \mathcal{S}(X) \rightarrow \mathcal{P}(X)$, where $\mathcal{S}(X)$ is the space of sequences in $X$ and $\mathcal{P}(X)$ is the space of parts of $X$. We will always assume that the limit operator is: (a) coherent, and so, that $\mathrm{L}(\sigma) \subset \mathrm{L}(\mathrm{K})$ where $\kappa, \sigma \in \mathcal{S}(X)$ and $\kappa$ is a subsequence of $\sigma$ (this will be denoted by $\kappa \subset \sigma$ ); and (b) finite-invariant ensuring that $\mathrm{L}(\sigma)=\mathrm{L}(\mathrm{K})$ if a common subsequence is obtained by deleting a finite number of terms in both sequences.

Any (coherent and finite-invariant) limit operator defines naturally a topology $\tau_{\mathrm{L}}$ on $X$ on the following way: a set $C$ is closed for $\tau_{L}$ if and only if $L(\sigma) \subset C$ for all sequence $\sigma \subset \mathrm{C}$. Such a topology is sequential, i.e., it is completely determined by the convergence of its sequences (a subset is closed if and only if it contains all its convergent sequences); this happens even if $\mathrm{L}(\sigma)$ only determines some of the possible limits of $\sigma$. Reciprocally, any sequential topology $\tau$ has associated a limit operator $L_{\tau}$ (its usual convergence) such that $\tau=\tau_{\mathrm{L}_{\tau}}$ (see [6, proposition 2.6]). Observe however that the previous limit operator is not the unique limit operator which determines the same topology $\tau$. Among the limits defining a concrete sequential topology $\tau$, it is always possible to choose one satisfying that $p \in \mathrm{L}\left(\{\mathrm{p}\}_{\mathfrak{n}}\right)$ for all $p \in M$, where $\{p\}_{\mathfrak{n}}$ denotes the constant sequence $p$. In the particular case when $\{p\}=\mathrm{L}\left(\{\mathrm{p}\}_{\mathfrak{n}}\right)$, we will say that the limit operator is idempotent. Finally, the pair $(X, L)$ will also represent the sequential topological space $\left(X, \tau_{L}\right)$.

In general, the limit operator $L$ does not determine the complete set of convergence points of a sequence $\sigma$ with the topology $\tau_{\mathrm{L}}$. In fact, the only implication which is always true is that:

$$
p \in \mathrm{L}(\sigma) \Longrightarrow \sigma \text { converges to } p \text { with the topology } \tau_{\mathrm{L}} .
$$

When the other implication is satisfied for all sequences, we will say that the limit operator is of first order. In general, there are not many results determining when a limit operator is of first order. In fact, in practical cases, the proof is done case by case, taking special care of "problematic" sequences. However, if we relax slightly the first order condition on L, we can obtain simply-to-check conditions which will be enough for our purposes. In this sense, let us introduce some definitions.

Definition 2.1. Let $\mathrm{X}$ be a space and $\mathrm{L}$ a limit operator defined on $\mathrm{X}$. Let us denote by $\tau_{\mathrm{L}}$ the associated sequential topology and let $\sigma \subset \mathrm{X}$ be a sequence. We will say that $\mathrm{L}$ is of first order for $\sigma$ if

$$
\mathrm{p} \in \mathrm{L}(\sigma) \Longleftrightarrow \sigma \text { converges to } \mathrm{p} \text { with the topology } \tau_{\mathrm{L}} \text {. }
$$

Additionally, we will say that $\mathrm{L}$ is of first order up to a subsequence for $\sigma$ (or first order UTS for short), if $\sigma$ has a subsequence $\mathrm{\kappa} \subset \sigma$ such that $\mathrm{L}$ is of first order for $\mathrm{\kappa}$. Finally, we will say that $\mathrm{L}$ is of first order UTS if it is of first order UTS for all sequence $\sigma \subset \mathrm{X}$.

The following result give us a sufficient condition to ensure when a limit operator is of first order for a given sequence.

Lemma 2.2. Consider (X, L) a sequential space with $\mathrm{L}$ idempotent. Let $\sigma$ be a sequence on $\mathrm{X}$ such that, for all subsequence $\mathrm{\kappa} \subset \sigma, \mathrm{L}(\mathrm{\kappa})=\mathrm{L}(\sigma)$. Assume additionally that $\mathrm{L}(\sigma)$ 
only contains a finite number of elements. Then, $\operatorname{cl}(\sigma)=\sigma \cup \mathrm{L}(\sigma)$, where $\operatorname{cl}(\sigma)$ denotes the topological closure of $\sigma$. In particular, $\mathrm{L}$ is of first order for $\sigma$.

Proof. The proof is quite straightforward and we include it here for the sake of completeness. Observe that the set $\mathrm{C}=\sigma \cup \mathrm{L}(\sigma) \subset \operatorname{cl}(\sigma)$ from (2.1), so the first assertion follows if we prove that $\mathrm{C}$ is closed. For this, let $\mathrm{K} \subset \mathrm{C}$ and let us prove that $\mathrm{L}(\mathrm{k}) \subset \mathrm{C}$. Recall that, due the finite number of elements in $L(\sigma)$, we have have two possibilities (up to a subsequence) for $\mathrm{K} \subset \mathrm{C}$ : or the sequence $\mathrm{K}$ is a subsequence of $\sigma$, and so, $\mathrm{L}(\mathrm{K})=\mathrm{L}(\sigma) \subset \mathrm{C}$; or $\kappa$ is constantly an element $p \in C$, and so, $L(\kappa)=\{p\} \subset C$. In both cases, $L(\kappa) \subset C$ and hence $\mathrm{C}$ is closed.

For the last assertion, that is, the first order character of $L$ on $\sigma$, let us assume that $\sigma \rightarrow p$. Again, we distinguish two cases:

- We can exclude a finite number of elements in $\sigma$ such that the refined sequence $\sigma^{\prime}$ does not contain $p$. As we are removing only a finite number of elements, $\mathrm{L}\left(\sigma^{\prime}\right)=\mathrm{L}(\sigma)$ and it follows from the first assertion that $\operatorname{cl}\left(\sigma^{\prime}\right)=\sigma^{\prime} \cup \mathrm{L}\left(\sigma^{\prime}\right)$. As $\sigma^{\prime} \rightarrow p$, we have that $p \in \sigma^{\prime} \cup \mathrm{L}\left(\sigma^{\prime}\right)$. From construction $\sigma^{\prime}$ does not contain $p$, so $p \in \mathrm{L}\left(\sigma^{\prime}\right)=\mathrm{L}(\sigma)$.

- Otherwise, we can construct a subsequence $\kappa$ of $\sigma$ with $\kappa=\{p\}_{n}$. In particular, $p \in \mathrm{L}\left(\{\mathrm{p}\}_{\mathfrak{n}}\right)=\mathrm{L}(\mathrm{K})=\mathrm{L}(\sigma)$ (recall that the last equality follows by hypothesis).

In conclusion, $p \in L(\sigma)$ and $L$ is of first order for $\sigma$.

Previous result give us a relatively simple way to determine when $L$ is of first order for a given sequence $\sigma$ (and so, to determine when $L$ is of first order) and it is usually enough in particular cases. However, we can go a step further on the search of a easily verifiable condition. For this, let us note that most of the results we will present on this paper require, not a complete control of the convergence of sequences, but the existence for any sequence of a subsequence sufficiently well behaved. This is make apparent in the following result which ensures continuity of a map between sequential spaces:

Proposition 2.3. Let $\mathrm{f}:(\mathrm{M}, \mathrm{L}) \rightarrow\left(\mathrm{N}, \mathrm{L}^{\prime}\right)$ be a map between sequential spaces $(\mathrm{M}, \mathrm{L})$ and $\left(\mathrm{N}, \mathrm{L}^{\prime}\right)$. The map $\mathrm{f}$ is continuous if for any sequence $\left\{\mathrm{p}_{\mathrm{n}}\right\}_{\mathrm{n}} \subset \mathrm{M}$ and $\mathrm{p} \in \mathrm{L}\left(\left\{\mathrm{p}_{\mathrm{n}}\right\}_{\mathfrak{n}}\right)$ there exists a subsequence $\left\{\mathrm{p}_{\mathrm{n}_{\mathrm{k}}}\right\}_{\mathrm{k}}$ of $\left\{\mathrm{p}_{\mathrm{n}}\right\}_{\mathrm{n}}$ such that $\mathrm{f}(\mathrm{p}) \in \mathrm{L}^{\prime}\left(\left\{\mathrm{f}\left(\mathrm{p}_{\mathrm{n}_{\mathrm{k}}}\right)\right\}_{\mathrm{k}}\right)$.

Proof. Let $C$ be a closed set in $\left(N, L^{\prime}\right)$, and let us show that $f^{-1}(C)$ is closed on $(M, L)$. Assume by contradiction that $f^{-1}(C)$ is not closed and so, from definition, that there exists a sequence $\sigma \subset f^{-1}(C)$ and a point $p \in M$ with $p \in L(\sigma) \backslash f^{-1}(C)$. From hypothesis, there exists a subsequence $\kappa \subset \sigma$ such that $f(p) \in L^{\prime}(f(\kappa))$. But $f(k) \subset C$, which is closed for the topology $\tau_{L^{\prime}}$. Therefore $f(p) \in C$, and so, $p \in f^{-1}(C)$, a contradiction.

This is one of the reasons why the condition of L being of first order UTS is specially interesting for us. Moreover, as we can see on the following result, it is possible to obtain the following sufficient conditions for the first order UTS, which is particularly simple to verify in practical cases: 
Lemma 2.4. Let $\mathrm{X}$ be any space with an idempotent limit operator $\mathrm{L}$ defined on it. Assume that $\# \mathrm{~L}(\bar{\sigma})<\infty$ for all sequence $\bar{\sigma} \subset \mathrm{X}$. Then, $\mathrm{L}$ is of first order UTS. ${ }^{1}$

Proof. Let $\sigma \subset \mathrm{X}$ be an arbitrary sequence. Observe that there are two (exclusive) possibilities for the sequence:

(a) for all subsequence $\mathrm{K} \subset \sigma, \mathrm{L}(\mathrm{\kappa})=\mathrm{L}(\sigma)$ or

(b) there exists $\mathrm{\kappa}_{1} \subset \sigma$ with $\mathrm{L}(\sigma) \subsetneq \mathrm{L}\left(\mathrm{\kappa}_{1}\right)$. In particular, $\# \mathrm{~L}\left(\mathrm{\kappa}_{1}\right) \geqslant \# \mathrm{~L}(\sigma)+1$.

In the first case, $\mathrm{L}$ is of first order for $\sigma$ according to lemma 2.2 and we are done. In the second case, we can repeat the same argument with $\kappa_{1}$ on the role of $\sigma$. Again there are two possibilities: either it ends in a finite number of iterations with a sequence $\kappa_{n_{0}}$ satisfying previous (a), hence, with $L$ being of first order for $\kappa_{n_{0}}$; or we obtain a chain of subsequences

$$
\kappa_{1} \supset K_{2} \supset \cdots \supset K_{n} \supset \cdots
$$

with $\# \mathrm{~L}\left(\kappa_{i+1}\right) \geqslant \# \mathrm{~L}\left(\kappa_{i}\right)+1$. However, this second posibility will lead us to the existence of a sequence with infinite limits, a contradiction. In fact, if $\kappa_{i}=\left\{x_{\mathfrak{n}}^{i}\right\}_{\mathfrak{n}}$ then the diagonal sequence $\left\{x_{n}^{\mathfrak{n}}\right\}_{\mathfrak{n}}$ satisfies:

$$
\cup_{i} \mathrm{~L}\left(\kappa_{i}\right) \subset \mathrm{L}\left(\left\{x_{n}^{n}\right\}_{n}\right)
$$

which implies that $\# \mathrm{~L}\left(\left\{x_{n}^{n}\right\}_{n}\right)=\infty$ due the increasing character of $\# \mathrm{~L}\left(\kappa_{i}\right)$. In conclusion, previous inductive process should end in a finite number of steps, obtaining a subsequence of $\sigma$ where $L$ is of first order.

Finally, let us review how sequential topologies behaves under a quotient. As it was proved on [20, remark 5.12], given a sequential space $(X, L)$ and an equivalence relation $\sim$ defined on it, the quotient topological space $X / \sim$ (with the induced topology) is again a sequential space. In fact, it is possible to give explicitly a limit operator $\mathrm{L}_{\mathrm{Q}}$ whose associated topology coincides with the quotient topology in $X / \sim$ in the following way:

$$
[x] \in \mathrm{L}_{\mathrm{Q}}\left(\left\{\left[x_{n}\right]\right\}_{\mathfrak{n}}\right) \Longleftrightarrow \exists x^{\prime} \in \mathfrak{i}^{-1}([x]), x_{n}^{\prime} \in \mathfrak{i}^{-1}\left(\left[x_{n}\right]\right) \forall n \in \mathbb{N}: x^{\prime} \in \mathrm{L}\left(\left\{x_{n}^{\prime}\right\}_{\mathfrak{n}}\right) .
$$

where $i: X \rightarrow X / \sim$ is the natural quotient projection and $[x],\left[x_{n}\right] \in X / \sim$. As it happens in the general case of topological spaces, the quotient topology of sequential spaces could not preserve the separability conditions of the original topological space. This is particularly interesting regarding the $T_{1}$ condition, which is translated on limit operators by the idempotent property (so points are closed with the associated sequential topology). As we will see on example 5.1, we can obtain a non idempotent limit operator $\mathrm{L}_{\mathrm{Q}}$ even when $\mathrm{L}$ is.

\footnotetext{
${ }^{1}$ We would like to thanks Prof. S. Harris who make us aware of this improvement for a previous version of the result.
} 


\subsection{C-boundary construction}

The causal completion was firstly introduced by Geroch, Kronheimer and Penrose in their seminal work [21]. The main idea for such a construction is to attach for any future-past inextensible timelike curve an ideal point characterized by the past-future of the curve. The original construction presents several problems mainly related with the topology considered. However, the notion of causal boundary and completion have been widely developed [2227 ] (see also the reviews in $[2,4]$ ), reaching a definition for the causal completion (named c-completion) fully satisfactory on [5].

Let us review some classical concepts of causal theory, referring the reader to [28] for further details and classical notation. Let $(\mathrm{V}, \mathfrak{g})$ be a connected, time-oriented Lorentz manifold. Denote by $\ll$ the chronological relation (respectively $\leqslant$ the causal relation), that is, $p \ll q(p \leqslant q)$ iff there exists a future-directed timelike (causal) curve from $p$ to $q$. In what follows, the spacetime $V$ will be considered strongly causal, and so, the intersections between the chronological future and past of points generate the topology in $V$. In particular, strong causality ensures also that $V$ is distinguishing, hence two different points $\mathrm{p}, \mathrm{q} \in \mathrm{V}$ have different future $\mathrm{I}^{+}(\mathrm{p}) \neq \mathrm{I}^{+}(\mathrm{q})$ and past $\mathrm{I}^{-}(\mathrm{p}) \neq \mathrm{I}^{-}(\mathrm{q})$.

A non-empty subset $P \subset V$ is called a past set if it coincides with its past, i.e., $\mathrm{P}=\mathrm{I}^{-}(\mathrm{P}):=\{\mathrm{p} \in \mathrm{V}: \mathrm{p} \ll \mathrm{q}$ for some $\mathrm{q} \in \mathrm{P}\}$. Let $\mathrm{S} \subset \mathrm{V}$ and define the common past of $S$ as $\downarrow S:=I^{-}(\{p \in V: \quad p \ll q \forall q \in S\})$. Observe that, from definition, the past and common past sets are open. A past set that cannot be written as the union of two proper past sets is called indecomposable past set, IP for short. An indecomposable past set $\mathrm{P}$ belongs to one of the following two categories: $\mathrm{P}$ can be expressed as the past of a point of the spacetime, i.e., $\mathrm{P}=\mathrm{I}^{-}(\mathrm{p})$ for some $\mathrm{p} \in \mathrm{V}$, and so, $\mathrm{P}$ is called proper indecomposable past set, PIP; or $\mathrm{P}=\mathrm{I}^{-}\left(\left\{x_{n}\right\}_{n}\right)$ for some inextensible future-directed chronological sequence $\left\{x_{\mathfrak{n}}\right\}_{\mathfrak{n}},{ }^{2}$ and then $\mathrm{P}$ is called terminal indecomposable past set, TIP. The dual notions of future set, common future, IF, PIF and TIF, are defined just by interchanging the roles of past and future in previous definitions.

The future causal completion is defined as the set of all indecomposable past sets IPs. As the manifold $V$ is distinguishing, the original manifold points $p \in V$ are naturally identified with their past $\mathrm{p} \equiv \mathrm{I}^{-}(\mathrm{p})$, and so, $\mathrm{V}$ is identified with the set of PIPs. Therefore, the future causal boundary $\hat{\partial} \mathrm{V}$ is defined as the set of all TIPs in $\mathrm{V}$, obtaining the following identifications:

$$
\mathrm{V} \equiv \mathrm{PIPs}, \quad \hat{\partial} \mathrm{V} \equiv \mathrm{TIPs}, \quad \hat{\mathrm{V}} \equiv \operatorname{IPs} .
$$

The future causal completion will be endowed with the future chronological topology $\hat{\tau}_{\mathrm{chr}}$, a sequential topology defined by the following limit operator: for $\sigma=\left\{P_{n}\right\}_{\mathfrak{n}} \subset \hat{V}$,

$$
\mathrm{P} \in \hat{\mathrm{L}}_{\mathrm{chr}}\left(\left\{\mathrm{P}_{n}\right\}_{n}\right) \Longleftrightarrow \mathrm{P} \subset \operatorname{LI}\left(\left\{\mathrm{P}_{n}\right\}_{n}\right) \text { and it is maximal in } \operatorname{LS}\left(\left\{\mathrm{P}_{n}\right\}_{n}\right) \text {. }
$$

Here by maximal we mean that no other $\mathrm{P}^{\prime} \in \hat{\mathrm{V}}$ satisfies the stated property and includes strictly P. The symbols LS and LI denotes superior and inferior limits for sets respectively,

\footnotetext{
${ }^{2}$ Here, by a future-directed chronological sequence $\left\{x_{n}\right\}_{n}$ we mean that $x_{n} \ll x_{n+1}$ for all $n$, see [29] for details on this approach of the causal boundary.
} 
which are defined in the following way: given a sequence $\left\{A_{n}\right\}_{n}$ of sets,

$$
\operatorname{LI}\left(\left\{A_{n}\right\}_{n}\right)=\cup_{m=1}^{\infty} \cap_{n=m}^{\infty} A_{n} \quad \operatorname{LS}\left(\left\{A_{n}\right\}_{n}\right)=\cap_{m=1}^{\infty} \cup_{n=m}^{\infty} A_{n}
$$

An analogous definition follows for the past causal completion by interchanging the roles of future and past sets. Hence,

$$
\mathrm{V} \equiv \mathrm{PIFs}, \quad \check{\partial} \mathrm{V} \equiv \mathrm{TIFs}, \quad \check{\mathrm{V}} \equiv \mathrm{IFs},
$$

and $\check{V}$ is endowed with the past chronological topology $\check{\tau}_{c h r}$ defined by a limit operator $\check{\mathrm{L}}_{c h r}$.

For the (total) c-boundary, we need to recall that some IPs and IFs represent naturally the same point of the completion. This is quite evident for PIPs and PIFs, where future and past sets can be identified if they are future and past of the same point respectively. However, previous identification is insufficient, as other indecomposable sets have to be identified. For this, let us define the so-called S-relation (introduced on [26]). Denote by $\hat{V}_{\emptyset}=\hat{V} \cup\{\emptyset\}$ and by $\check{V}_{\emptyset}=\check{V} \cup\{\emptyset\}$. The S-relation $\sim_{S}$ is defined in $\hat{V}_{\emptyset} \times \check{V}_{\emptyset}$ as follows. A pair $(P, F) \in \hat{V} \times \check{V}$ is S-related if

$$
\mathrm{P} \sim_{\mathrm{S}} \mathrm{F} \Longleftrightarrow \begin{cases}\mathrm{P} & \text { is included and is a maximal IP into } \downarrow \mathrm{F} \\ \mathrm{F} & \text { is included and is a maximal IF into } \uparrow \mathrm{P} .\end{cases}
$$

As proved by Szabados [26], the past and future of a point $p \in V$ are $S$-related, $\mathrm{I}^{-}(p) \sim S$ $\mathrm{I}^{+}(\mathrm{p})$, and these are the unique S-relations (according to our definition (2.4)) involving proper indecomposable sets. We also define that $P \sim_{S} \emptyset$ (respectively $\emptyset \sim_{S} F$ ) if $P$ (respectively F) is a non-empty, necessarily terminal indecomposable past (respectively future) set that is not S-related by (2.4) to any other indecomposable set (note that $\emptyset$ is never S-related to itself).

Now, we can introduce the notion of c-completion. At the point set level, and following the idea of Marolf and Ross [10], the c-completion is formed by S-related pairs of indecomposable sets

$$
\overline{\mathrm{V}}:=\left\{(\mathrm{P}, \mathrm{F}) \in \hat{\mathrm{V}}_{\emptyset} \times \check{\mathrm{V}}_{\emptyset}: \mathrm{P} \sim_{\mathrm{S}} \mathrm{F}\right\} .
$$

Every point $p \in V$ of the manifold is naturally identified with its corresponding pair $\left(\mathrm{I}^{-}(\mathrm{p}), \mathrm{I}^{+}(\mathrm{p})\right)$, so $\mathrm{V}$ can be (and will be) considered a subset of $\bar{V}$. The $c$-boundary is then defined as $\partial \vee=\bar{V} \backslash V$. The boundary points can be classified in three different classes, that we will define now for future reference.

Definition 2.5. Let $(\mathrm{P}, \mathrm{F}) \in \partial \mathrm{V}$ be an arbitrary point on the $c$-boundary. We will say that $(\mathrm{P}, \mathrm{F})$ is a timelike boundary point if both components are non empty $\mathrm{P} \neq \emptyset \neq \mathrm{F}$. The point is a lightlike boundary point if one of the components is empty and, in the case $\mathrm{P} \neq \emptyset$ (respectively $\mathrm{F} \neq \emptyset$ ) there exists $\mathrm{P}^{\prime}$ (respectively $\mathrm{F}^{\prime}$ ) a proper indecomposable set such that $\mathrm{P} \subsetneq \mathrm{P}^{\prime}$ (respectively $\left.\mathrm{F} \subsetneq \mathrm{F}^{\prime}\right)$. Finally, in the remainder case, a terminal set $\mathrm{P}$ (respectively $\mathrm{F})$ not contained in any other IP (respectively IF), is a spatial boundary point. ${ }^{3}$

\footnotetext{
${ }^{3}$ Observe that the definition of spatial point has also sense for partial boundaries.
} 
The chronological relation on $\mathrm{V}$ is also extended to the c-completion in the following way (by abuse of notation, we denote the chronological relation on $\bar{V}$ with the same symbol): given two points $(P, F),\left(P^{\prime}, F^{\prime}\right) \in \bar{V}$

$$
(\mathrm{P}, \mathrm{F}) \ll\left(\mathrm{P}^{\prime}, \mathrm{F}^{\prime}\right) \Longleftrightarrow \mathrm{F} \cap \mathrm{P}^{\prime} \neq \emptyset .
$$

It is not possible to obtain in general a explicit expression for the causal relation, as we have done for the chronological relation. However, it is known that any chronological relation has naturally associated a causal relation $\leqslant$ (see [30, definition 2.22] for details).

Remark 2.6. Now that we have defined the chronological relation in $\bar{V}$, we can understand better the terminology introduced in definition 2.5. As it is clear, if a boundary point $(\mathrm{P}, \mathrm{F})$ is timelike (and so, with both components non empty), then $(\mathrm{P}, \mathrm{F})$ lies in the past (respectively future) of any point $y \in F$ (respectively $y \in P$ ). Otherwise, we know that $P$ or $F$ should be empty. Let us assume that $F=\emptyset$ (the other case will be analogous). Now observe that if $(P, \emptyset)$ is lightlike, then there exists another point $\left(P^{\prime}, F^{\prime}\right) \in \bar{V}$ with $P \subsetneq P^{\prime}$. It is clear that previous points cannot be timelike related according to (2.6), however it follows that $(\mathrm{P}, \emptyset) \leqslant\left(\mathrm{P}^{\prime}, \mathrm{F}^{\prime}\right)$ according to [31, section 6.4], being natural to assume that both points are horismotically related. Finally, if $(P, \emptyset)$ is a spatial boundary point, then no pair $\left(\mathrm{P}^{\prime}, \mathrm{F}^{\prime}\right) \in \overline{\mathrm{V}}$ will satisfy that $(\mathrm{P}, \emptyset) \leqslant\left(\mathrm{P}^{\prime}, \mathrm{F}^{\prime}\right)$.

Finally, $\bar{V}$ is endowed with the chronological topology $\tau_{\mathrm{chr}}$, a sequential topology associated to the following limit operator (known as the chronological limit): for a sequence $\sigma=\left\{\left(P_{n}, F_{n}\right)\right\}_{\mathfrak{n}} \subset \bar{V}$, define

$$
\mathrm{L}_{\mathrm{chr}}(\sigma):=\left\{(\mathrm{P}, \mathrm{F}) \in \overline{\mathrm{V}}: \begin{array}{l}
\mathrm{P} \in \hat{\mathrm{L}}_{\mathrm{chr}}\left(\left\{\mathrm{P}_{n}\right\}_{\mathfrak{n}}\right) \text { if } \mathrm{P} \neq \emptyset \\
\mathrm{F} \in \check{\mathrm{L}}_{\mathrm{chr}}\left(\left\{\mathrm{F}_{n}\right\}_{\mathfrak{n}}\right) \text { if } \mathrm{F} \neq \emptyset
\end{array}\right\} .
$$

It is important to recall, as it will be used later, that due the definition of the S-relation between terminal sets, the definition of the chronological limit is simplified when both terminal sets on the limit are non empty (see [5, lemma 3.15]). Concretely:

Proposition 2.7. Let $\left\{\left(\mathrm{P}_{\mathrm{n}}, \mathrm{F}_{\mathrm{n}}\right)\right\}_{\mathrm{n}}$ be a sequence of pairs in $\overline{\mathrm{V}}$ and assume that $\mathrm{P} \sim_{\mathrm{S}} \mathrm{F}$ with $\mathrm{P} \neq \emptyset \neq \mathrm{F}$. If $\mathrm{P} \subset \operatorname{LI}\left(\left\{\mathrm{P}_{n}\right\}_{n}\right)$ and $\mathrm{F} \subset \operatorname{LI}\left(\left\{\mathrm{F}_{n}\right\}_{n}\right)$ then $(\mathrm{P}, \mathrm{F}) \in \mathrm{L}_{c h r}\left(\left\{\left(\mathrm{P}_{n}, \mathrm{~F}_{n}\right)\right\}_{n}\right)$.

The following result will summarize the main properties of the c-completion endowed with the chronological relation and topology (see [5, theorem 3.27] and its proof).

Theorem 2.8. Let $(\mathrm{V}, \mathfrak{g})$ be a strongly causal Lorentzian manifold and $\overline{\mathrm{V}}$ its causal completion endowed with the chronological structure induced by (2.6) and the topology induced from the chronological limit (2.7). Then:

(i) The inclusion $\mathrm{V} \hookrightarrow \overline{\mathrm{V}}$ is continuous. Moreover, the restriction of the chronological limit on $\mathrm{V}$ is a first order limit operator.

(ii) Let $\left\{x_{n}\right\}_{n} \subset \mathrm{V}$ be a future (respectively past) chronological sequence Then,

$$
\mathrm{L}_{\mathrm{chr}}\left(\left\{\mathrm{x}_{\mathfrak{n}}\right\}_{\mathfrak{n}}\right)=\left\{(\mathrm{P}, \mathrm{F}) \in \overline{\mathrm{V}}: \mathrm{P}=\mathrm{I}^{-}\left(\left\{\mathrm{x}_{\mathfrak{n}}\right\}_{\mathfrak{n}}\right)\left(\text { respectively } \mathrm{F}=\mathrm{I}^{+}\left(\left\{\mathrm{x}_{\mathrm{n}}\right\}_{\mathfrak{n}}\right)\right)\right\}
$$


(iii) The c-completion is complete: for any past terminal set $\mathrm{P}$ (respectively future terminal set $\mathrm{F}$ ) there exists $\mathrm{F}$ (respectively $\mathrm{P}$ ) such that $(\mathrm{P}, \mathrm{F}) \in \overline{\mathrm{V}}$. In particular, any inextensible timelike curve $\gamma$ on $\mathrm{V}$ (respectively any inextensible chronological sequence $\left\{x_{\mathfrak{n}}\right\}_{n}$ on $\left.\mathrm{V}\right)$ has an endpoint in $\overline{\mathrm{V}}$.

(iv) The sets $\mathrm{I}^{ \pm}((\mathrm{P}, \mathrm{F}))$ are open for all $(\mathrm{P}, \mathrm{F}) \in \overline{\mathrm{V}}$.

(v) $\overline{\mathrm{V}}$ is a $\mathrm{T}_{1}$ topological space.

\subsection{Spacetime covering projections: the causal ladder and main properties}

Let us consider that we have an action on $\mathrm{V}$ given by a group $\mathrm{G}$ of isometric maps. ${ }^{4}$

$$
\begin{aligned}
\mathrm{G} \times \mathrm{V} & \rightarrow \mathrm{V} \\
(\mathrm{g}, \mathrm{p}) & \rightarrow \mathrm{g} p .
\end{aligned}
$$

We will always assume that the action preserves time-orientation, and acts freely and properly discontinuously, where the latter means: (a) for each $p \in \mathrm{V}$, there exists a neighborhood $\mathrm{U}$ such that $\mathrm{g} \mathrm{U} \cap \mathrm{U}=\emptyset$ for all $\mathrm{g} \in \mathrm{G} \backslash\{\mathrm{e}\}$ and; (b) for $\mathrm{p}_{1}, \mathrm{p}_{2} \in \mathrm{V}$ there are neighbourhoods $\mathrm{U}_{1}$ and $\mathrm{U}_{2}$ such that $\mathrm{g} \mathrm{U}_{1} \cap \mathrm{U}_{2}=\emptyset$ for all $\mathrm{g} \in \mathrm{G}$.

Previous conditions over the action let us ensure that the quotient space $M=V / G$ is also a Lorentzian manifold with the induced metric (which will be denoted by an abuse of notation as $\mathfrak{g}$ ). The canonical projection to the quotient space, denoted by $\pi: \mathrm{V} \rightarrow \mathrm{M}$, will be called a spacetime covering projection. The following result let us understand clearly the relation between the chronological relation on $M$ and $V$ (the same result follows for causal relations, see [19, proposition 1.1]).

Proposition 2.9. Let us consider $\pi: \mathrm{V} \rightarrow \mathrm{M}$ a spacetime covering projection. Then:

- If $\mathrm{p}, \mathrm{q} \in \mathrm{V}$ satisfy that $\mathrm{p} \ll \mathrm{q}$, then $\pi(\mathrm{p}) \ll \pi(\mathrm{q})$.

- If $\mathrm{x}, \mathrm{y} \in \mathrm{M}$ satisfy that $\mathrm{x} \ll \mathrm{y}$, then for any $\mathrm{p}, \mathrm{q} \in \mathrm{V}$ with $\pi(\mathrm{p})=\mathrm{x}$ and $\pi(\mathrm{q})=\mathrm{y}$, there exists an element $\mathrm{g} \in \mathrm{G}$ such that $\mathrm{p} \ll \mathrm{g} \mathrm{q}$.

As it is clear, previous result is key to understanding the relation between the causal structures of both, $V$ and $M$. From a global viewpoint, it is possible to characterize all the stages of the well known causal ladder on $M$ (see [30]) in terms of the global causal structure of $\mathrm{V}$. We will summarize in the following result some of such characterizations, which proofs can be found on [19, Props. 1.2, 1.3 and 1.4].

Theorem 2.10. Let $\pi: \mathrm{V} \rightarrow \mathrm{M}$ be a spacetime covering projection with group $\mathrm{G}$. Then:

(CL1) $\mathrm{M}$ is non-totally vicious if, and only if, there exists $\mathrm{p}, \mathrm{q} \in \mathrm{V}$ with $\pi(\mathrm{p})=\pi(\mathrm{q})$ and $p \nless q$.

\footnotetext{
${ }^{4}$ The results of this paper can be obtained considering a group $\mathrm{G}$ of conformal maps, but we will consider isometric actions for simplicity.
} 
(CL2) $\mathrm{M}$ is chronological if, and only if, for all $\mathrm{p}, \mathrm{q} \in \mathrm{V}$ with $\pi(\mathrm{p})=\pi(\mathrm{q}), \mathrm{p} \nless \mathrm{q}$.

(CL3) $\mathrm{M}$ is causal if, and only if, for all $\mathrm{p}, \mathrm{q} \in \mathrm{V}$ with $\pi(\mathrm{p})=\pi(\mathrm{q}), \mathrm{p} \not \mathbf{q}$.

(CL4) $\mathrm{M}$ is strongly causal if, and only if, for all $\mathrm{p} \in \mathrm{V}$ there is a fundamental neighbourhood system $\left\{\mathrm{U}_{\mathrm{n}}\right\}$ for $\mathrm{p}$ such that for each $\mathrm{n}$, no causal curve can have one endpoint in $\mathrm{U}_{\mathrm{n}}$ and another endpoint in a component $\mathrm{U}_{n}^{\prime}$ of $\pi^{-1}\left(\pi\left(\mathrm{U}_{\mathrm{n}}\right)\right)$ unless $\mathrm{U}_{\mathrm{n}}^{\prime}=\mathrm{U}_{\mathrm{n}}$ and the curve remains wholly within $\mathrm{U}_{\mathrm{n}}$.

(CL5) $\mathrm{M}$ is globally hyperbolic if, and only if,

(CL5-1) $\mathrm{V}$ is globally hyperbolic,

(CL5-2) every point $\mathrm{p} \in \mathrm{V}$ has a fundamental neighbourhood system as in (CL4) and

(CL5-3) for any $\mathrm{p} \in \mathrm{V}$, for all $\mathrm{p} \ll \mathrm{q}$, $\mathrm{J}^{+}(\mathrm{p}) \cap \pi^{-1}(\pi(\mathrm{q}))$ is finite.

Let us remark that in all previous cases, a global causal condition on $M$ (i.e., the assumption of a stage in the causal ladder) implies a stronger global condition on $\mathrm{V}$. However at this point, it is not clear for us at what extent the same property follows for the rest of the causal ladder (particularly with causally continuous and causally simple), being necessary a detailed study on such cases. However, that study is out the scope of this paper.

\section{Partial Boundaries under the action of the group}

In this section, we will study the behaviour of the future causal completion under the action of an isometry group $\mathrm{G}$, being the past case completely analogous. Let us begin with a point in the future completion of $V$, that is, an indecomposable set $\bar{P}=\mathrm{I}^{-}\left(\left\{\mathrm{p}_{n}\right\}_{n}\right)$, where $\left\{\mathrm{p}_{n}\right\}_{n}$ is a future-directed chronological sequence. As the group $\mathrm{G}$ acts by isometries in $\mathrm{V}$, the sequence $\left\{x_{n}\right\}_{n}$ with $\pi\left(p_{n}\right)=x_{n}$ is also future-directed and chronological (proposition 2.9), hence, it defines the indecomposable set $\mathrm{P}=\mathrm{I}^{-}\left(\left\{x_{n}\right\}_{n}\right)$ in $M$. Therefore, the projection $\pi$ extends naturally to the corresponding partial completions on the following way:

$$
\begin{aligned}
\hat{\pi}: \hat{V} & \rightarrow \hat{\mathrm{M}} \\
\overline{\mathrm{P}}=\mathrm{I}^{-}\left(\left\{\mathrm{p}_{\mathrm{n}}\right\}_{\mathfrak{n}}\right) & \rightarrow \mathrm{P}=\mathrm{I}^{-}\left(\left\{x_{n}\right\}_{\mathfrak{n}}\right) .
\end{aligned}
$$

We will say that an indecomposable set $\overline{\mathrm{P}} \in \hat{\mathrm{V}}$ is a lift of $\mathrm{P}$ if $\hat{\pi}(\overline{\mathrm{P}})=\mathrm{P}$.

Previous map is always surjective, as any future-directed chronological sequence $\left\{x_{n}\right\}_{n}$ in $M$ can be lifted to a future-directed chronological sequence $\left\{p_{n}\right\}_{n}$ in $V$ (by proposition 2.9). However, the map is not injective in general, as previous lift is not unique. For instance, if $\left\{p_{n}\right\}_{n}$ is a lift of $\left\{x_{n}\right\}_{n},\left\{g p_{n}\right\}_{n}$ (for any $g \in G$ ) is also a lift of the same sequence. Even more, the pre-image of a terminal set $\mathrm{P}$ can be easily characterized. Let us denote by $\overline{\mathrm{P}}=\mathrm{I}^{-}\left(\left\{\mathrm{p}_{n}\right\}_{n}\right)$, where $\left\{\mathrm{p}_{n}\right\}_{n}$ denotes one fixed lift of $\left\{x_{n}\right\}_{n}$. It follows that

$$
\hat{\pi}^{-1}(P)=\cup_{g \in G} g \bar{P},
$$


i.e., the pre-image of $\mathrm{P}$ is the union of what we are going to call the G-orbit of $\overline{\mathrm{P}}$ in $\hat{\mathrm{V}}$, which is the set $\{\mathbf{g} \overline{\mathrm{P}}\}_{\mathbf{g} \in \mathrm{G}}$. The left inclusion is straightforward, as $\hat{\pi}(\mathrm{g} \overline{\mathrm{P}})=\mathrm{P}$ for all $\mathbf{g} \in \mathrm{G}$. For the right one, take a point $x \in P$ and let $p \in V$ be a point such that $\pi(p)=x$. As $x \in P$, there exists $n$ big enough such that $x \ll x_{n}$. Hence, proposition 2.9 ensures that $p \ll g p_{n} \in g \bar{P}$ for some $g \in G$.

Convention 3.1. From this point, there are some useful conventions that we will use along the paper. For instance, the points on $\mathrm{M}$ will be denoted by $\mathrm{x}, \mathrm{y}, z$, while the points on $\mathrm{V}$ will be denoted by $\mathrm{p}, \mathrm{q}, \mathrm{r}$. Moreover, unless stated otherwise, we will always assume that $\pi(\mathrm{p})=\mathrm{x}, \pi(\mathrm{q})=\mathrm{y}$ and $\pi(\mathrm{r})=z$.

For any chronological sequence $\left\{x_{n}\right\}_{n}$ in $\mathrm{M}$ (respectively, an indecomposable set $\mathrm{P}$ ), we will consider a fixed lift on $\mathrm{V}$ denoted by $\left\{\mathrm{p}_{\mathrm{n}}\right\}_{\mathrm{n}}$ (respectively $\overline{\mathrm{P}}$ ). As an abuse of notation, we will use the same symbol $\mathrm{I}^{ \pm}$for future/past of sets when there is no confusion if we are in $\mathrm{M}$ or $\mathrm{V}$.

Finally, and in order to compute both, partial and c-boundary, we will assume from this point that $\mathrm{M}$ is strongly causal and so that $\mathrm{V}$ satisfies the condition described on theorem 2.10 (CL4).

The projection $\hat{\pi}$ let us define an equivalence relation on $\hat{V}$ : two indecomposable sets $\overline{\mathrm{P}}_{1}, \overline{\mathrm{P}}_{2} \in \hat{\mathrm{V}}$ are $\hat{\mathrm{G}}$-related, $\overline{\mathrm{P}}_{1} \sim_{\hat{\mathrm{G}}} \overline{\mathrm{P}}_{2}$, if and only if both terminal sets projects onto the same $\mathrm{P} \in \hat{\mathrm{M}}$, i.e., $\hat{\pi}\left(\overline{\mathrm{P}}_{1}\right)=\hat{\pi}\left(\overline{\mathrm{P}}_{2}\right)$. Of course, previous relation lead us to a bijection between the quotient space $\hat{V} / \hat{G}\left(\equiv \hat{V} / \sim_{\hat{G}}\right)$ and $\hat{M}$. However, the following two observations are in order: on the one hand, one could expect naively that for any two terminal sets with $\overline{\mathrm{P}}_{1} \sim_{\hat{\mathrm{G}}} \overline{\mathrm{P}}_{2}$, there exists $\mathrm{g} \in \mathrm{G}$ such that $\overline{\mathrm{P}}_{1}=g \overline{\mathrm{P}}_{2}$. Nonetheless, the following simple example shows that such a property is not true: consider the two-dimensional Minkowski spacetime, $\mathbb{L}^{2}$, with the action

$$
\begin{aligned}
\mathbb{Z} \times \mathbb{L}^{2} & \rightarrow \mathbb{L}^{2} \\
(z,(x, t)) & \rightarrow(x+z, t)
\end{aligned}
$$

defined on it. The lightlike line $\gamma(t)=(t, t)$ defines naturally a terminal set $\bar{P} \neq \mathrm{V}$. The $\mathbb{Z}$-orbit of $\overline{\mathrm{P}}$ is the complete spacetime $\mathbb{L}^{2}$, so both $\mathbb{L}^{2}$ and $\overline{\mathrm{P}}$ will be $\hat{\mathbb{Z}}$-related, but no element of the group send one to the other.

In any case, there are several examples where previous property is naturally satisfied. For instance, the same previous group action will satisfy the property if it is restricted to $\mathrm{V}=\mathbb{R} \times(\mathrm{a}, \mathrm{b}) \subset \mathbb{L}^{2}$. In fact, we can construct even more physically appealing examples for Robertson Walker spacetimes satisfying the integral conditions (6.2) (recall that, in terms of causality, Robertson Walker models satisfying such a integral conditions behave like Lorentzian product spaces with finite time interval, see [17]). This motivates the following definition:

Definition 3.2. A spacetime covering projection $\pi: \mathrm{V} \rightarrow \mathrm{M}$ is future tame if given two terminal sets $\overline{\mathrm{P}}_{1}, \overline{\mathrm{P}}_{2}$ with $\overline{\mathrm{P}}_{1} \sim_{\hat{\mathrm{G}}} \overline{\mathrm{P}}_{2}$ there exists $\mathrm{g} \in \mathrm{G}$ such that $\overline{\mathrm{P}}_{1}=\mathrm{g} \overline{\mathrm{P}}_{2}$. 
On the other hand, the induced map is not well behaved at the topological level. In fact, Harris shows in the last example of [19] that $\hat{\pi}$ is not, in general, continuous (see also example 5.1 for details).

The rest of this section is devoted to make a deep comparison between the topologies of $\hat{M}$ and $\hat{V} / \hat{G}$, where the latter has the induced quotient topology. Let us first fix some notation. As we have mention on section $2.2, \hat{M}$ and $\hat{V}$ will be endowed with the future chronological topology, which is defined by a limit operator (2.3). In order to differentiate both limits, we will denote by $\hat{\mathrm{L}}_{M}$ the future chronological limit on $\hat{M}$ and, accordingly, $\hat{\mathrm{L}}_{V}$ the limit on $\hat{V}$. The quotient topology on $\hat{V} / \hat{G}$ is also a sequential topology (see section 2.1) and it is defined from a limit operator (2.2) which will be denoted here by $\hat{\mathrm{L}}_{\hat{\mathrm{G}}}$. Finally, recall that the map $\hat{\pi}$ induces a bijective map $\hat{\jmath}$ between $\hat{V} / \hat{G}$ and $\hat{M}$ which makes the following diagram commutative:

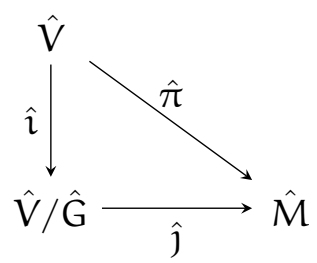

being $\hat{\imath}: \hat{V} \rightarrow \hat{V} / \hat{G}$ is the usual projection.

Previous $\hat{\jmath}$ map is always open. In order to prove this, we require the following technical lemma.

Lemma 3.3. Consider a sequence $\sigma=\left\{\mathrm{P}_{\mathrm{n}}\right\}_{\mathfrak{n}} \subset \hat{\mathrm{M}}$ and a point $\mathrm{P} \in \hat{\mathrm{M}}$ such that $\mathrm{P} \subset$ $\operatorname{LI}\left(\left\{\mathrm{P}_{\mathfrak{n}}\right\}_{\mathfrak{n}}\right)$. For $\overline{\mathrm{P}}$ a fixed lift of $\mathrm{P}$, there exist lifts $\overline{\mathrm{P}}_{\mathrm{n}}$ of $\mathrm{P}_{\mathfrak{n}}$ such that $\overline{\mathrm{P}} \subset \operatorname{LI}\left(\left\{\overline{\mathrm{P}}_{\mathrm{n}}\right\}_{\mathfrak{n}}\right)$.

Proof. Let us begin by taking $\left\{\overline{\mathrm{P}}_{n}\right\}_{n}$ some fixed lifts of $\left\{\mathrm{P}_{n}\right\}_{n}$. Denote also by $\left\{\mathrm{p}_{n}\right\}_{n}$ and $\left\{x_{n}\right\}_{n}$ future chronological chains defining $\bar{P}$ and $P$ respectively and satisfying that $\pi\left(p_{n}\right)=x_{n}$ (as stated in Convention 3.1). As $P \subset \operatorname{LI}\left(\left\{P_{n}\right\}_{n}\right)$, for any element $x_{n}$ there exists $m_{n} \in \mathbb{N}$ (that we can consider strictly increasing on $n$ ) such that, for all $m \geqslant m_{n}$, $x_{\mathrm{n}} \in \mathrm{P}_{\mathrm{m}}$. In particular, and due to proposition 2.9, we can ensure the existence of $\mathrm{g} \in \mathrm{G}$ in such a way that $p_{n} \in g \bar{P}_{m}$. Then, for $m \geqslant m_{n}$, let us denote by $G(n, m) \subset G$ the non-empty subset defined in the following way:

$$
\mathrm{G}(\mathrm{n}, \mathrm{m}):=\left\{\mathrm{g} \in \mathrm{G}: \mathrm{p}_{\mathrm{n}} \in \mathrm{g} \overline{\mathrm{P}}_{\mathrm{m}}\right\}
$$

Let us make a straightforward (but necessary) observation about previous sets. As $p_{n} \ll p_{n+1}$, for $m \geqslant m_{n+1}\left(\geqslant m_{n}+1\right)$,

$$
\mathrm{G}(\mathrm{n}+1, \mathrm{~m}) \subset \mathrm{G}(\mathrm{n}, \mathrm{m}) .
$$

Now, for each $m_{n} \leqslant m<m_{n+1}$, let us consider a group element $g_{m} \in G(n, m)$ and consider the sequence $\left\{g_{m} \bar{P}_{m}\right\}_{m}$ (for $m<m_{1}$, just consider $g_{m}=e$, the identity). Now, let us show that previous sequence is the desired, that is, $\overline{\mathrm{P}} \subset \operatorname{LI}\left(\left\{\boldsymbol{g}_{\mathrm{m}} \overline{\mathrm{P}}_{\mathrm{m}}\right\}_{\mathrm{m}}\right)$. In fact, 
for any $n \in \mathbb{N}$, consider $m \geqslant m_{n}$ and denote by $k \in \mathbb{N} \cup\{0\}$ the natural ensuring that $m_{n+k+1}>m \geqslant m_{n+k}$. Then, from the choice of $\left\{g_{m}\right\}_{m}$ and (3.3), we have that:

$$
g_{m} \in G(n+k, m) \subset G(n+k-1, m) \subset \cdots \subset G(n, m) .
$$

In conclusion, from (3.2) we deduce that $p_{n} \in g_{m} \bar{P}_{m}$ for all $m \geqslant m_{n}$, and the result follows.

Proposition 3.4. Let $\pi: \mathrm{V} \rightarrow \mathrm{M}$ be a spacetime covering projection and $\hat{\pi}: \hat{\mathrm{V}} \rightarrow \hat{\mathrm{M}}$ the extended map on the corresponding partial completions. The induced map $\hat{\jmath}: \hat{\mathrm{V}} / \hat{\mathrm{G}} \rightarrow \hat{\mathrm{M}}$ is open.

Proof. Let us prove that the map $\hat{\jmath}^{-1}$ is continuous by using proposition 2.3. For this, consider a sequence $\sigma=\left\{\mathrm{P}_{n}\right\}_{n} \subset \hat{M}$ and a point $\mathrm{P} \in \hat{\mathrm{L}}_{M}(\sigma)$, and let us show that $\hat{\jmath}^{-1}(P) \in \hat{L}_{\hat{G}}\left(\hat{\jmath}^{-1}(K)\right)$ for some subsequence $\kappa \subset \sigma$. Recall that, from the definitions of $\hat{L}_{\hat{G}}$ and $\hat{\jmath}^{-1}$, this is the same that show the existence of lifts $\bar{P}_{n}$ and $\bar{P}$ of $P_{n}$ and $P$ respectively such that $\overline{\mathrm{P}} \in \hat{\mathrm{L}}_{\mathrm{V}}\left(\left\{\overline{\mathrm{P}}_{\mathrm{n}_{k}}\right\}_{k}\right)$ for some subsequence $\left\{\overline{\mathrm{P}}_{\mathrm{n}_{k}}\right\}_{k} \subset\left\{\overline{\mathrm{P}}_{n}\right\}_{n}$.

First observe that, by using previous lemma, we can find lifts $\bar{P}_{n}$ and $\bar{P}$ of $P_{n}$ and $P$ respectively such that $\overline{\mathrm{P}} \subset \operatorname{LI}\left(\left\{\overline{\mathrm{P}}_{n}\right\}_{n}\right)$. If $\overline{\mathrm{P}}$ is maximal in $\operatorname{LS}\left(\left\{\overline{\mathrm{P}}_{n}\right\}_{n}\right)$, then we have that $\overline{\mathrm{P}} \in \hat{\mathrm{L}}_{\mathrm{V}}\left(\left\{\overline{\mathrm{P}}_{\mathrm{n}}\right\}_{\mathfrak{n}}\right)$, and we are done.

Otherwise, take $\overline{P^{\prime}}$ a maximal set in $\operatorname{LS}\left(\left\{\overline{\mathrm{P}}_{\mathfrak{n}}\right\}_{\mathfrak{n}}\right)$ containing $\overline{\mathrm{P}}$, and let $\left\{\mathrm{p}_{n}^{\prime}\right\}_{\mathfrak{n}}$ be a future chronological sequence generating $\overline{\mathrm{P}^{\prime}}$. As $\overline{\mathrm{P}^{\prime}} \subset \operatorname{LS}\left(\left\{\overline{\mathrm{P}}_{n}\right\}_{n}\right)$, it is possible to find a strictly increasing subsequence $\left\{k_{n}\right\}_{n}$ such that $p_{n}^{\prime} \in \bar{P}_{k_{n}}$ for all $n$. Then, it follows readily that $\overline{\mathrm{P}^{\prime}} \in \hat{\mathrm{L}} \mathrm{V}\left(\left\{\overline{\mathrm{P}}_{\mathrm{n}_{k}}\right\}_{k}\right)$. Now observe that the sets $\mathrm{P}^{\prime}=\hat{\pi}\left(\overline{\mathrm{P}^{\prime}}\right)$ and $\mathrm{P}_{n_{k}}=\hat{\pi}\left(\overline{\mathrm{P}}_{\mathrm{n}_{k}}\right)$ satisfy the following chain ( $\hat{\pi}$ preserves contentions)

$$
\mathrm{P} \subset \mathrm{P}^{\prime} \subset \operatorname{LI}\left(\left\{\mathrm{P}_{\mathrm{n}_{\mathrm{k}}}\right\}_{\mathrm{k}}\right)
$$

But as $\mathrm{P} \in \hat{\mathrm{L}}_{M}\left(\left\{\mathrm{P}_{n_{k}}\right\}_{k}\right)$, it follows that $\mathrm{P}=\mathrm{P}^{\prime}$ (recall the maximal character on (2.3)) and so that $\overline{P^{\prime}}$ is also a lift of $P$.

In both cases, and up to a subsequence, we show the existence of lifts $\left\{\bar{P}_{n}\right\}_{n}$ and $\bar{P}$ with $\bar{P} \in \hat{L}_{V}\left(\left\{\bar{P}_{n}\right\}_{\mathfrak{n}}\right)$, and then the continuity of $\hat{\jmath}^{-1}$ follows from proposition 2.3 .

Remark 3.5. Previous proof shows in particular that for all $P \in \hat{L}_{M}\left(\left\{P_{n}\right\}_{n}\right)$, there exist lifts $\bar{P}$ and $\bar{P}_{n}$ of $P$ and $P_{n}$ respectively with $\bar{P} \in \hat{L}_{V}\left(\left\{\bar{P}_{n_{k}}\right\}_{k}\right)$ for some subsequence $\left\{\bar{P}_{n_{k}}\right\}_{k}$ of $\left\{\overline{\mathrm{P}}_{n}\right\}_{n}$.

As we have already pointed out, the map $\hat{\jmath}$ is not continuous in general. If we look into the details of example 5.1, we see that the non-continuity is related with the following situation: there exists a (non-necessarily chronological) sequence $\left\{P_{n}\right\}_{n} \subset \hat{M}$ admitting two different lifts such that (i) both lifted sequences are convergent and (ii) the projection of one limit point contains strictly the other. As we will see, such a situation represent, essentially, the only cases where continuity of $\hat{\pi}$ can fail, so it is convenient to give a proper name for it: 
Definition 3.6. Let $\pi: \mathrm{V} \rightarrow \mathrm{M}$ a spacetime covering projection and $\hat{\mathrm{V}}, \hat{\mathrm{M}}$ the corresponding future causal completions of $\mathrm{V}$ and $\mathrm{M}$. We will say that a sequence $\sigma=\left\{\mathrm{P}_{\mathfrak{n}}\right\}_{\mathfrak{n}} \subset \hat{\mathrm{M}}$ has future divergent lifts if there exist two lifts $\left\{\overline{\mathrm{P}}_{n}\right\}_{n},\left\{{\overline{\mathrm{P}^{\prime}}}_{n}\right\}_{n} \subset \hat{\mathrm{V}}$ of $\sigma$ and two points $\overline{\mathrm{P}}, \overline{\mathrm{P}^{\prime}} \in \hat{\mathrm{V}}$ such that:

(i) $\overline{\mathrm{P}} \in \hat{\mathrm{L}}_{V}\left(\left\{\overline{\mathrm{P}}_{n}\right\}_{\mathfrak{n}}\right)$ and $\overline{\mathrm{P}^{\prime}} \in \hat{\mathrm{L}}_{\mathrm{V}}\left(\left\{\overline{\mathrm{P}}_{\mathrm{n}}\right\}_{\mathfrak{n}}\right)$.

(ii) $\hat{\pi}(\overline{\mathrm{P}}) \subsetneq \hat{\pi}\left(\overline{\mathrm{P}^{\prime}}\right)$.

If there exists no such a sequence on $\hat{\mathrm{M}}$, we will just say that $\mathrm{M}$ does not admit sequences with future divergent lifts.

As a side remark, observe that the concept of divergent lifts is quite related with the topological structure of the G-orbits in $\hat{V}$. In fact, we can prove the following result:

Proposition 3.7. Let $\pi$ be future tame. Then, the G-orbits of $\hat{\mathrm{V}}$ are closed if and only if $\mathrm{M}$ does not admit constant sequences with divergent lifts.

Proof. For the right implication let $\mathrm{P} \in \hat{\mathrm{M}}$ and $\overline{\mathrm{P}} \in \hat{\mathrm{V}}$ with $\hat{\pi}(\overline{\mathrm{P}})=\mathrm{P}$. Observe that by the tame condition every lift of the constant sequence $\{P\}_{n}$ has the form $\left\{g_{n} \bar{P}\right\}_{n}$ where $g_{n} \in G$. So, if $\overline{P^{\prime}} \in \hat{V}$ is such that $\overline{P^{\prime}} \in \hat{L_{V}}\left(\left\{g_{n} \bar{P}\right\}_{n}\right)$ then the closedness of the G-orbit ensures that $\overline{\mathrm{P}^{\prime}}=g_{0} \overline{\mathrm{P}}$ for some $\mathrm{g}_{0} \in \mathrm{G}$. Therefore, $\{\mathrm{P}\}_{\mathrm{n}}$ admits no divergent lifts as condition (ii) in definition 3.6 cannot be fulfilled.

For the left one, assume that $M$ admits no constant sequence with divergent lifts and let us prove that the G-orbits in $\hat{V}$ are closed. Let $\mathrm{P}, \overline{\mathrm{P}}, \overline{\mathrm{P}^{\prime}}$ and $\left\{\mathrm{g}_{\mathrm{n}}\right\}_{n}$ as in previous implication. As $M$ admits no constant sequence with divergent lifts, then necessarily it follows that $\hat{\pi}\left(\overline{P^{\prime}}\right)=\mathrm{P}$. Moreover, as $\pi$ is future tame, then there exists $g_{0} \in \mathrm{G}$ such that $\overline{\mathrm{P}^{\prime}}=g_{0} \overline{\mathrm{P}}$, and so, $\overline{\mathrm{P}^{\prime}}$ belongs to the G-orbit $\{\mathrm{g} \overline{\mathrm{P}}\}_{\mathrm{g} \in \mathrm{G}}$ and the G-orbit is closed.

The optimality of previous result follows from example 5.5 where it is shown a case where $M$ admits no constant sequence with divergent lifts but the $\mathrm{G}$-orbits are not closed.

Our main technical result on this section is the following characterization of the continuity of $\hat{\pi}$ (up to the first order UTS condition):

Proposition 3.8. Let $\left\{\overline{\mathrm{P}}_{\mathrm{n}}\right\}_{\mathfrak{n}}$ be a sequence whose projection $\left\{\mathrm{P}_{\mathrm{n}}\right\}_{\mathfrak{n}}$ does not admit divergent lifts. Then,

$$
\overline{\mathrm{P}} \in \hat{\mathrm{L}}_{\mathrm{V}}\left(\left\{\overline{\mathrm{P}}_{\mathrm{n}}\right\}_{\mathrm{n}}\right) \Rightarrow \mathrm{P}:=\hat{\pi}(\overline{\mathrm{P}}) \in \hat{\mathrm{L}}_{\mathrm{M}}\left(\left\{\mathrm{P}_{\mathrm{n}}\right\}_{\mathfrak{n}}\right) .
$$

In particular, if $\mathrm{M}$ does not admit sequences with future divergent lifts, the map $\hat{\pi}$ is continuous. Conversely, if the map $\hat{\pi}$ is continuous and additionally the future chronological limit $\hat{\mathrm{L}}_{\mathrm{M}}$ on $\hat{\mathrm{M}}$ is of first order UTS, then there are no sequences with divergent lifts.

Proof. Let $\bar{\sigma}=\left\{\overline{\mathrm{P}}_{\mathrm{n}}\right\}_{\mathrm{n}}$ be a sequence as in the first statement of the proposition, and consider $\overline{\mathrm{P}} \in \hat{\mathrm{L}}_{\mathbf{V}}(\bar{\sigma})$. By recalling that $\hat{\pi}$ preserves contentions, we deduce that $\mathrm{P} \subset \operatorname{LI}\left(\left\{\mathrm{P}_{n}\right\}_{\mathfrak{n}}\right)$. If $\mathrm{P}$ is maximal among the IPs in $\operatorname{LS}\left(\left\{P_{n}\right\}_{n}\right)$, then $P \in \hat{L}_{M}\left(\left\{P_{n}\right\}_{n}\right)$ and we are done.

So, let us assume by contradiction that $\mathrm{P}$ is not maximal on the $\operatorname{LS}\left(\left\{\mathrm{P}_{\mathrm{n}}\right\}_{\mathrm{n}}\right)$. Consider $\mathrm{P}^{\prime}$ a maximal IP on $\operatorname{LS}\left(\left\{\mathrm{P}_{n}\right\}_{n}\right)$ containing strictly P. From the definition of the superior limit, 
and up to a subsequence, we can assume that $\mathrm{P}^{\prime} \subset \operatorname{LI}\left(\left\{\mathrm{P}_{n}\right\}_{\mathfrak{n}}\right)$, and so, that $\mathrm{P}^{\prime} \in \hat{\mathrm{L}}_{M}\left(\left\{\mathrm{P}_{n}\right\}_{\mathfrak{n}}\right)$. Now, recalling remark 3.5, we ensure that $P_{n}$ and $P^{\prime}$ admit lifts $\overline{P^{\prime}} n$ and $\overline{P^{\prime}}$ such that $\overline{P^{\prime}} \in \hat{L}_{V}\left(\left\{\overline{P^{\prime}} n_{k}\right\}_{k}\right)$. Summarizing, the sequence $\left\{P_{n_{k}}\right\}_{k}$ admits two lifts $\left\{\bar{P}_{n_{k}}\right\}_{k}$ and $\left\{{\overline{P^{\prime}}}_{n_{k}}\right\}_{k}$ converging to $\overline{\mathrm{P}}$ and $\overline{\mathrm{P}^{\prime}}$ respectively, where $\mathrm{P}=\hat{\pi}(\overline{\mathrm{P}}) \subsetneq \hat{\pi}\left(\overline{\mathrm{P}^{\prime}}\right)=\mathrm{P}^{\prime}$. That is to say, $\left\{\mathrm{P}_{\mathrm{n}_{\mathrm{k}}}\right\}_{\mathrm{k}}$ admits future divergent lifts, a contradiction. In conclusion, $P \in \hat{L}_{M}\left(\left\{P_{n}\right\}_{n}\right)$. Moreover, if $M$ does not admit sequences with divergent lifts, $\hat{\pi}$ is continuous (recall proposition 2.3).

For the final assertion, assume that $\hat{\mathrm{L}}_{M}$ is of first order UTS and that there exists a sequence $\sigma=\left\{P_{n}\right\}_{n} \subset \hat{M}$ with divergent lifts. Let $\left\{\bar{P}_{n}\right\}_{n},\left\{{\overline{P^{\prime}}}_{n}\right\}_{n}$ be two sequences in $\hat{V}$ and $\bar{P}, \overline{P^{\prime}}$ two terminal sets as in definition 3.6. Assume by contradiction that $\hat{\pi}$ is continuous. In particular, we have that $\left\{\mathrm{P}_{n}\right\}_{\mathfrak{n}}$ (the projection by $\hat{\pi}$ of both sequences $\left\{\overline{\mathrm{P}}_{n}\right\}_{n}$ and $\left\{{\overline{P^{\prime}}}_{n}\right\}_{n}$ ) converges to $P$ and $P^{\prime}$. As $\hat{L}_{M}$ is of first order UTS, we can assume that (up to a subsequence) $\hat{\mathrm{L}}_{M}$ is of first order for $\left\{\mathrm{P}_{n}\right\}_{n}$, and so, that $\mathrm{P}, \mathrm{P}^{\prime} \in \hat{\mathrm{L}}_{M}\left(\left\{\mathrm{P}_{n}\right\}_{n}\right)$. But this is a contradiction with the definition of $\hat{\mathrm{L}}_{M}(2.3)$ (concretely the maximal character of the limit points) and the fact that $\mathrm{P} \subsetneq \mathrm{P}^{\prime}$ (definition 3.6 (ii)). Therefore, the map $\hat{\pi}$ cannot be continuous.

There are several ways to prove the non-existence of sequences with divergent lifts. For instance, we can impose conditions on the causality of the boundary (re-obtaining [19, theorem 3.4])

Corollary 3.9. If $\hat{M}$ has only spatial future boundary points (see definition 2.5 and Footnote 3), then $\hat{\pi}$ is continuous, and so, $\hat{\jmath}$ is a homeomorphism between $\hat{\mathrm{M}}$ and $\hat{\mathrm{V}} / \hat{\mathrm{G}}$.

Proof. Assume by contradiction that $\hat{\pi}$ is not continuous and so, from previous result, that there exists a sequence $\sigma \subset \hat{M}$ admitting divergent lifts. Let $\bar{\sigma}, \overline{\sigma^{\prime}}$ be two sequences in $\hat{V}$ and $\overline{\mathrm{P}}, \overline{\mathrm{P}^{\prime}}$ be two points in $\hat{\mathrm{V}}$ as in definition 3.6. As $\hat{\mathrm{M}}$ only contains spatial future boundary points, no IP can contain a TIP. Hence, from (ii) in definition 3.6, we deduce that $\mathrm{P}=\mathrm{I}^{-}(\chi)$ for some $x \in M$, and then, $\overline{\mathrm{P}}=\mathrm{I}^{-}(\mathrm{p})$ for some point $\mathrm{p} \in \mathrm{V}$. As $\pi: \mathrm{V} \rightarrow \mathrm{M}$ is continuous and the future chronological topology preserves the manifold topology (which follows from theorem 2.8, (i)), we have that $\mathrm{P} \in \hat{\mathrm{L}}_{\mathbf{M}}\left(\left\{\mathrm{P}_{\mathrm{n}}\right\}_{\mathfrak{n}}\right)$. Finally from (i) and (ii) in definition 3.6 we have that $\mathrm{P} \subsetneq \mathrm{P}^{\prime} \subset \mathrm{LI}\left(\left\{\mathrm{P}_{n}\right\}_{\mathfrak{n}}\right)$, in contradiction with the maximality on (2.3).

Another possibility is to impose conditions over the topology of the future causal completion. In this case, we have also need to impose the finiteness of the group G:

Corollary 3.10. Consider $\pi: \mathrm{V} \rightarrow \mathrm{M}$ a spacetime covering with associated group $\mathrm{G}$. Assume that $\mathrm{G}$ is finite and that $\hat{\mathrm{V}}$ is Hausdorff. Then, $\hat{\pi}$ is continuous, and so, $\hat{\jmath}$ is a homeomorphism.

Proof. As we will see in the forthcoming sections, if $\mathrm{G}$ is finite then $\pi$ is future tame (see lemma 4.18). Hence, let us consider two sequences $\left\{\overline{\mathrm{P}}_{n}\right\}_{\mathfrak{n}},\left\{\overline{\mathrm{P}}_{n}\right\}_{\mathfrak{n}} \subset \hat{\mathrm{V}}$ and two points $\overline{\mathrm{P}}, \overline{\mathrm{P}^{\prime}} \in \hat{\mathrm{V}}$ with $\overline{\mathrm{P}} \in \hat{\mathrm{L}}_{V}\left(\left\{\overline{\mathrm{P}}_{n}\right\}_{\mathfrak{n}}\right)$ and $\overline{\mathrm{P}^{\prime}} \in \hat{\mathrm{L}}_{\mathrm{V}}\left(\left\{\overline{\mathrm{P}}_{n}\right\}_{\mathfrak{n}}\right)$ and such that $\hat{\pi}\left(\overline{\mathrm{P}}_{\mathfrak{n}}\right)=\hat{\pi}\left(\overline{\mathrm{P}}_{n}\right)$. Our aim is to prove that $\hat{\pi}(\overline{\mathrm{P}})=\hat{\pi}\left(\overline{\mathrm{P}^{\prime}}\right)$ as then no sequence with divergent lifts can exists.

Recalling the tameness of $\pi$, there exists a sequence $\left\{g_{n}\right\}_{n} \subset G$ such that ${\overline{P^{\prime}}}_{n}=g_{n} \bar{P}_{n}$. Due the assumption that $\mathrm{G}$ is finite, we can assume (up to a subsequence) that $\mathrm{g}_{\mathrm{n}} \equiv \mathrm{g}_{\mathrm{0}}$ for 
all $n$ and some constant $g_{0} \in G$. Therefore, $\bar{P} \in \hat{L}_{V}\left(\left\{\bar{P}_{n}\right\}_{n}\right)$ and $\overline{P^{\prime}} \in \hat{L}_{V}\left(\left\{g_{0} \bar{P}_{n}\right\}_{n}\right)$. From the first inclusion and the fact that $G$ acts by isometries, we deduce that $g_{0} \bar{P}$ also belongs to $\hat{L}_{V}\left(\left\{g_{0} \bar{P}_{n}\right\}_{n}\right)$ and recalling that $\hat{V}$ is Hausdorff (and so, for any sequence $\sigma, \hat{L}_{V}(\sigma)$ can contain at most one element, recall (2.1)), it follows that $g_{0} \bar{P}=\overline{P^{\prime}}$, as desired.

\subsection{Proof of theorem 1.1}

Assertion (i) follows from proposition 3.4, while (ii) from proposition 3.8. The last assertion is proved in corollaries 3.9 and 3.10 .

\section{The C-completion under the action of the group}

Once we have determined the requirements to ensure the well behaviour of the partial boundaries, we are in conditions to study the (total) c-completion. As a first step, we will deal with the projection and lift of points of the corresponding c-completions, in order to define an extension $\bar{\pi}: \bar{V} \rightarrow \bar{M}$. Later, we will study the properties of such a map at both, the chronological and the topological level.

\subsection{Point set level}

Let us begin by considering $\overline{\mathrm{P}} \in \hat{\mathrm{V}}$ and $\overline{\mathrm{F}} \in \check{\mathrm{V}}$ two non empty indecomposable sets which are S-related, so $(\overline{\mathrm{P}}, \overline{\mathrm{F}}) \in \overline{\mathrm{V}}$; and let us study when the projections of each component of the pair of such terminal sets are $S$-related. Of course, if these sets correspond to the past and future of a point $p \in V$, their projections will correspond to the past and future of the projection $x=\pi(p) \in M$ (and so, they are $S$-related). Therefore, we can assume that $\bar{P}$ and $\bar{F}$ are terminal sets. Let us denote by $\left\{p_{n}\right\}_{n}$ and $\left\{q_{n}\right\}_{n}$ the corresponding inextensible (future and past respectively) chronological sequences defining them. From the definition of the S-relation and the chronological limits, it follows that $\overline{\mathrm{P}} \in \hat{\mathrm{L}}_{\mathbf{V}}\left(\left\{\mathrm{I}^{-}\left(\mathbf{q}_{\mathbf{n}}\right)\right\}_{\mathfrak{n}}\right)$ (see Thm 2.8 (ii)). If the past chronological sequence $\left\{y_{n}\right\}_{n}$ (projection of $\left\{q_{n}\right\}_{n}$ ) does not admit future divergent lifts, then proposition 3.8 ensures that $P:=\hat{\pi}(\overline{\mathrm{P}}) \in \hat{\mathrm{L}}_{M}\left(\left\{\mathrm{I}^{-}\left(\mathrm{y}_{\mathfrak{n}}\right)\right\}_{\mathfrak{n}}\right)$. Then, taking into account that the past chronological sequence $\left\{y_{n}\right\}_{n}$ determines $F:=\check{\pi}(\bar{F})$, we obtain that $\mathrm{P} \subset \downarrow \mathrm{F}$ and it is maximal inside such a subset (see (2.3)). Analogously, assuming that the future chronological chain $\left\{x_{\mathfrak{n}}\right\}_{\mathfrak{n}}$ does not admit past divergent lifts, we can prove that $\mathrm{F} \subset \uparrow \mathrm{P}$ and it is maximal, so we have that:

Proposition 4.1. Let $\pi: \mathrm{V} \rightarrow \mathrm{M}$ be a spacetime covering projection. Assume that $\mathrm{M}$ does not admit an inextensible sequence $\left\{x_{\mathfrak{n}}\right\}_{\mathfrak{n}} \subset M$ which is either past-directed chronological with future divergent lifts or future-directed chronological with past divergent lifts. If $(\overline{\mathrm{P}}, \overline{\mathrm{F}}) \in$ $\overline{\mathrm{V}}$ with $\overline{\mathrm{P}} \neq \emptyset \neq \overline{\mathrm{F}}$, then $(\mathrm{P}, \mathrm{F}) \in \overline{\mathrm{M}}$, where $\mathrm{P}=\hat{\pi}(\overline{\mathrm{P}})$ and $\mathrm{F}=\check{\pi}(\overline{\mathrm{F}})$.

Previous condition for the future and past sequences is fulfilled in strongly regular cases as globally hyperbolic models, where inextesible past- (respectively future-)directed chronological sequences has no future (respectively past) limit. But of course, there will be other (not so regular) cases, as the one showed in corollary 4.23 (including for instance some Robertson-Walker models with an appropriate group action, see section 6) or the one in example 5.4, where the condition is naturally fulfilled. 
At the point set level, previous proposition is the only case where points are well projected in general. In fact, examples 5.2 and 5.3 show cases of points in $\bar{V}$ with no natural projection in $\bar{M}$. Moreover, these examples also show that the lifts of points from $\bar{M}$ are not, in general, well behaved either. Concretely, as we can see in example 5.3, the point $\left(P_{2}, \emptyset\right)$ has no natural lift in $\bar{V}$. The only possible candidate is the point $\left(\bar{P}_{2}, \bar{F}\right)$, but $\left(\mathrm{P}_{2}, \emptyset\right) \neq\left(\hat{\pi}\left(\overline{\mathrm{P}}_{2}\right), \check{\pi}(\overline{\mathrm{F}})\right)$, evenmore this last point does not belong to $\overline{\mathrm{M}}$.

However, if we characterize the conditions under which the lift of points $(P, F) \in \bar{M}$ with both components non empty are well defined, then we will be in conditions to define the projection between $\bar{V}$ and $\bar{M}$.

Proposition 4.2. Consider a point $(\mathrm{P}, \mathrm{F}) \in \overline{\mathrm{M}}$ with $\mathrm{P} \neq \emptyset \neq \mathrm{F}$. The point $(\mathrm{P}, \mathrm{F})$ has a lift in $\overline{\mathrm{V}}$, i.e., a pair $(\overline{\mathrm{P}}, \overline{\mathrm{F}}) \in \overline{\mathrm{V}}$ with $\mathrm{P}=\hat{\pi}(\overline{\mathrm{P}})$ and $\mathrm{F}=\check{\pi}(\overline{\mathrm{F}})$ if and only if there exist lifts $\overline{\mathrm{P}^{\prime}}$ and $\overline{\mathrm{F}^{\prime}}$ of $\mathrm{P}$ and $\mathrm{F}$ respectively such that $\overline{\mathrm{P}^{\prime}} \subset \downarrow \overline{\mathrm{F}^{\prime}}$ (or, equivalently, $\overline{\mathrm{F}^{\prime}} \subset \uparrow \overline{\mathrm{P}^{\prime}}$ ).

Proof. The right implication is trivial, so we only need to focus on the left one, that is, consider a point $(P, F) \in \bar{M}$ and suppose that there exist lifts $\overline{P^{\prime}}$ and $\overline{F^{\prime}}$ such that $\overline{P^{\prime}} \subset \downarrow \overline{F^{\prime}}$ (and so, with $\overline{\mathrm{F}^{\prime}} \subset \uparrow \overline{\mathrm{P}^{\prime}}$ ). We can ensure then the existence of an IP $\overline{\mathrm{P}}$ with $\overline{\mathrm{P}^{\prime}} \subset \overline{\mathrm{P}}$ and maximal among the indecomposable sets contained in $\downarrow \overline{F^{\prime}}$. Recalling that the projection is well behaved with contentions, we deduce that $\mathrm{P} \subset \hat{\pi}(\overline{\mathrm{P}}) \subset \downarrow F$. However $\mathrm{P} \sim_{S} F$, so the maximality on (2.4) implies that $\mathrm{P}=\hat{\pi}(\overline{\mathrm{P}})$.

Reasoning in the same way with $\overline{F^{\prime}} \subset \uparrow \bar{P}$, we can prove that there exists $\bar{F}$ with $\check{\pi}(\overline{\mathrm{F}})=\mathrm{F}$ and being a maximal IP contained in $\uparrow \overline{\mathrm{P}}$. In conclusion, $\overline{\mathrm{P}} \sim_{\mathrm{S}} \overline{\mathrm{F}}$ and the pair $(\overline{\mathrm{P}}, \overline{\mathrm{F}})$ belongs to $\overline{\mathrm{V}}$. Moreover, from construction $\hat{\pi}(\overline{\mathrm{P}})=\mathrm{P}$ and $\check{\pi}(\overline{\mathrm{F}})=\mathrm{F}$, as desired.

Remark 4.3. Recall that previous proof does not imply that the initial $\overline{\mathrm{P}^{\prime}}$ and $\overline{\mathrm{F}^{\prime}}$ are S-related, but that there exist others indecomposable sets $S$-related $\bar{P}$ and $\bar{F}$ such that: (a) $\overline{\mathrm{P}^{\prime}} \subset \overline{\mathrm{P}}, \overline{\mathrm{F}^{\prime}} \subset \overline{\mathrm{F}}$ and $(\mathrm{b}) \hat{\pi}(\overline{\mathrm{P}})=\hat{\pi}\left(\overline{\mathrm{P}^{\prime}}\right)$ and $\check{\pi}(\overline{\mathrm{F}})=\check{\pi}\left(\overline{\mathrm{F}^{\prime}}\right)$.

Now, we are ready to extend the projection to the c-completions. However, the definition of the projection is far more technical than the partial cases. The main problem here is the existence of different candidates for the projection of pairs $(\bar{P}, \emptyset)$ and $(\emptyset, \bar{F})$, and no reason to prioritize one of the candidates over the other. This will be reflected on the existence of different extensions of $\pi$ (depending on the choice we made for the projection) for the general case. Nonetheless, as we will see along this section, all the possible definitions will share the same properties. Moreover, all the ambiguity in the choice of an extension will disappear under some additional properties such as tameness or finite chronology (see section 4.4).

Let $(\bar{P}, \emptyset) \in \partial V$ be a point in the c-boundary and let us analyse the possible projections that such a point can have on $\partial M$ (an analogous study can be made for $(\emptyset, \bar{F})$ ). The first natural candidate to consider (taking the corresponding projection of each component) is the pair $(P, \emptyset)$ with $P=\hat{\pi}(\bar{P})$. However, as shown by example 5.5 , it is not necessarily true that $\mathrm{P} \sim_{S} \emptyset$. In fact, another pair $\left(\overline{\mathrm{P}^{\prime}}, \overline{\mathrm{F}^{\prime}}\right)$ with $\overline{\mathrm{P}^{\prime}} \neq \emptyset \neq \overline{\mathrm{F}^{\prime}}$ and with $\hat{\pi}(\overline{\mathrm{P}})=\hat{\pi}\left(\overline{\mathrm{P}^{\prime}}\right)$ can exist. If the projection of the components is well behaved under the $S$-relation (for example, as in proposition 4.1), then $\mathrm{P}=\hat{\pi}\left(\overline{\mathrm{P}^{\prime}}\right) \sim_{S} \check{\pi}\left(\overline{\mathrm{F}^{\prime}}\right)$. Therefore, it seems more natural to define the projection of $(\overline{\mathrm{P}}, \emptyset)$ as the projection (by components) of $\left(\overline{\mathrm{P}^{\prime}}, \overline{\mathrm{F}^{\prime}}\right)$ instead of $(\overline{\mathrm{P}}, \emptyset)$. 
Nonetheless, previous process does not give an unique way to define such a projection. This is shown in example 5.6, where we have three points $(\overline{\mathrm{P}}, \emptyset),\left(\overline{\mathrm{P}^{\prime}}, \overline{\mathrm{F}^{\prime}}\right),\left(\overline{\mathrm{P}^{\prime \prime}}, \overline{\mathrm{F}^{\prime \prime}}\right) \in \partial \mathrm{V}$ with $\mathrm{P}=\hat{\pi}(\overline{\mathrm{P}})=\hat{\pi}\left(\overline{\mathrm{P}^{\prime}}\right)=\hat{\pi}\left(\overline{\mathrm{P}^{\prime \prime}}\right)$ but also satisfying that $\check{\pi}\left(\overline{\mathrm{F}^{\prime}}\right) \neq \check{\pi}\left(\overline{\mathrm{F}^{\prime \prime}}\right)$. Both points $\left(\overline{\mathrm{P}^{\prime}}, \overline{\mathrm{F}^{\prime}}\right),\left(\overline{\mathrm{P}^{\prime \prime}}, \overline{\mathrm{F}^{\prime \prime}}\right)$ share the same properties, having no argument to prioritize one over the other. So, a choice has to be made and different projections between $\bar{V}$ and $\bar{M}$ appear.

In order to formalize previous process for the definition of the extended projection, let us define $\sim \mathrm{G}_{0}$ as a relation between pairs satisfying that $(\overline{\mathrm{P}}, \emptyset) \sim \mathrm{G}_{0}\left(\overline{\mathrm{P}^{\prime}}, \overline{\mathrm{F}^{\prime}}\right)$ (respectively $\left.(\emptyset, \overline{\mathrm{F}})) \sim \mathrm{G}_{0}\left(\overline{\mathrm{P}^{\prime}}, \overline{\mathrm{F}^{\prime}}\right)\right)$ if $\hat{\pi}(\overline{\mathrm{P}})=\hat{\pi}\left(\overline{\mathrm{P}^{\prime}}\right)$ (respectively $\check{\pi}(\overline{\mathrm{F}})=\check{\pi}\left(\overline{\mathrm{F}^{\prime}}\right)$ ). Then, define a map $\alpha: \bar{V} \rightarrow \bar{V}$ as

$$
\alpha((\bar{P}, \bar{F}))=\left\{\begin{array}{l}
\text { if some component is empty and there is some }\left(\overline{\mathrm{P}^{\prime}}, \overline{\mathrm{F}^{\prime}}\right) \sim \mathrm{G}_{\mathrm{O}}(\overline{\mathrm{P}}, \overline{\mathrm{F}}), \\
\text { a choice of one of this }\left(\overline{\mathrm{P}^{\prime}}, \overline{\mathrm{F}^{\prime}}\right) ; \\
\text { otherwise }(\overline{\mathrm{P}}, \overline{\mathrm{F}}) .
\end{array}\right.
$$

The existence of such a map is always ensured, but it is not in general unique as it depends on the selected element $\left(\overline{\mathrm{P}^{\prime}}, \overline{\mathrm{F}^{\prime}}\right)$. Once a map $\alpha$ is chosen, we are in conditions to define the extended projection.

Definition 4.4. Consider $\pi: \mathrm{V} \rightarrow \mathrm{M}$ be a spacetime covering projection induced by an action of a group $\mathrm{G}$ and let $\alpha$ be a choice as defined on previous paragraph. Then we define $\bar{\pi}_{\alpha}: \bar{V} \rightarrow \hat{M}_{\emptyset} \times \check{M}_{\emptyset}$ given by $\bar{\pi}_{\alpha}=\bar{\pi} \circ \alpha$, where

$$
\bar{\pi}: \overline{\mathrm{V}} \rightarrow \hat{\mathrm{M}}_{\emptyset} \times \check{\mathrm{M}}_{\emptyset}, \quad \bar{\pi}((\overline{\mathrm{P}}, \overline{\mathrm{F}}))=(\hat{\pi}(\overline{\mathrm{P}}), \check{\pi}(\overline{\mathrm{F}}))
$$

and we are defining $\hat{\pi}(\emptyset)=\emptyset$ and $\check{\pi}(\emptyset)=\emptyset$.

Remark 4.5. (a) Let us emphasize that the definition of $\alpha$ is nothing but a technical requirement in order to define the extension of the projection, and its concrete definition and properties will not affect the results from this point (see for instance the discussion in example 5.6). Therefore, and in order to simplify the notation, we will drop the subindex $\alpha$ on the definition of $\bar{\pi}_{\alpha}$, always assuming that a map $\alpha$ has been fixed from the beginning.

(b) It is also worth mentioning at this point that, in our main results, we have to include additional hypothesis as tameness or finite chronology (see definition 4.14), which will imply that there are no pairs $(\overline{\mathrm{P}}, \emptyset)$ and $\left(\overline{\mathrm{P}^{\prime}}, \overline{\mathrm{F}^{\prime}}\right)$ in $\overline{\mathrm{V}}$ with $\overline{\mathrm{P}^{\prime}} \neq \emptyset \neq \overline{\mathrm{F}^{\prime}}$ and $(\overline{\mathrm{P}}, \emptyset) \sim_{\mathrm{G}_{0}}\left(\overline{\mathrm{P}^{\prime}}, \overline{\mathrm{F}^{\prime}}\right)$ (with analogous version for the future, see lemma 4.7 and proposition 4.21 ). In these cases, $\alpha$ becomes the identity and so $\bar{\pi}_{\alpha}=\bar{\pi}$. Along the paper we will emphasize these situations by saying that the extended projection $\bar{\pi}$ is univocally determined.

Previous construction give us a reasonable way to define an extension for the spacetime covering projection $\pi$. However, as shown in example 5.3, such a map does not restrict properly to $\bar{M}$ because $\bar{\pi}((\bar{P}, \bar{F}))$ could not belong to $\bar{M}$. Yet, we can overcome this problem under the assumptions of Props. 4.1 and 4.2.

Proposition 4.6. If we assume that the points ( $\mathrm{P}, \mathrm{F}) \in \overline{\mathrm{M}}$ with $\mathrm{P} \neq \emptyset \neq \mathrm{F}$ have lifts in $\bar{V}$ (see proposition 4.2) and that $M$ does not admit an inextensible sequence $\left\{x_{n}\right\}_{n} \subset$ $\mathrm{M}$ which is either past-directed chronological with future divergent lifts or future-directed chronological with past divergent lifts, then $\bar{\pi}$ restricts properly to $\bar{M}$ and it is surjective. 
Proof. Let us begin by showing that $\bar{\pi}$ restricts properly to $\bar{M}$. Take $(\bar{P}, \bar{F}) \in \bar{V}$ an arbitrary point and let us consider $\bar{\pi}((\overline{\mathrm{P}}, \overline{\mathrm{F}}))$. Observe that there are essentially two possibilities for the projection: or it has both components non empty, or has one empty component. The former case follows if the initial point $(\overline{\mathrm{P}}, \overline{\mathrm{F}})$ has both components non empty or if one component is empty (without loss of generalization $\overline{\mathrm{F}}=\emptyset$ ) but there exists $\left(\overline{\mathrm{P}^{\prime}}, \overline{\mathrm{F}^{\prime}}\right)$ with both components non empty $G_{0}$ related to $(\bar{P}, \emptyset)$. In any such cases proposition 4.1 ensures that $\bar{\pi}((\overline{\mathrm{P}}, \overline{\mathrm{F}})) \in \overline{\mathrm{M}}$

In the latter, no point $\left(\overline{P^{\prime}}, \overline{F^{\prime}}\right)$ with both components non empty can be $\sim_{G_{0}}$ related with $(\bar{P}, \bar{F})$. In particular, one of the components of the point should be empty, say $\bar{F}=\emptyset$ (the other case is analogous). In this case, $\bar{\pi}((\bar{P}, \emptyset))=(P, \emptyset)$, and so, we have to prove that $\mathrm{P} \sim_{\mathrm{S}} \emptyset$. If not, from the completeness of the c-completion (recall theorem 2.8 (iii)), the terminal set $P$ should be $S$-related with a terminal set $F \neq \emptyset$, determining the point $(\mathrm{P}, \mathrm{F}) \in \overline{\mathrm{M}}$. From the hypothesis, there are non empty lifts $\overline{\mathrm{P}^{\prime}}$ and $\overline{\mathrm{F}^{\prime}}$ of $\mathrm{P}$ and $\mathrm{F}$ such that $\left(\overline{\mathrm{P}^{\prime}}, \overline{\mathrm{F}^{\prime}}\right) \in \overline{\mathrm{V}}$. However, we have that $\hat{\pi}(\overline{\mathrm{P}})=\hat{\pi}\left(\overline{\mathrm{P}^{\prime}}\right)$, and so, that $(\overline{\mathrm{P}}, \emptyset) \sim \mathrm{G}_{0}\left(\overline{\mathrm{P}^{\prime}}, \overline{\mathrm{F}^{\prime}}\right)$, a contradiction. In conclusion, $P \sim_{S} \emptyset$ and the projection restricts properly to $\bar{M}$.

For the surjectivity, consider $(P, F) \in \bar{M}$. If $(P, F)$ has both components non empty, then by hypothesis admits a lift on $\bar{V}$ which projects on it. Otherwise, assume without loss of generality that $F=\emptyset$ and take $\bar{P}$ any lift of $P$. From completeness of the c-completion, there exists $\overline{\mathrm{F}}$ such that $(\overline{\mathrm{P}}, \overline{\mathrm{F}}) \in \overline{\mathrm{V}}$. Moreover $\overline{\mathrm{F}}$ has to be empty as, otherwise, recalling that $\bar{\pi}$ restricts properly to $\overline{\mathrm{M}}, \mathrm{P} \sim_{S} \check{\pi}(\overline{\mathrm{F}})$ (which is not possible as $\mathrm{P} \sim_{S} \emptyset$ ). Hence, any point $(\overline{\mathrm{P}}, \overline{\mathrm{F}}) \in \overline{\mathrm{V}}$ with $\hat{\pi}(\overline{\mathrm{P}})=\mathrm{P}$ has $\overline{\mathrm{F}}=\emptyset$ and, from the definition of $\bar{\pi}$, we deduce that $\bar{\pi}((\bar{P}, \emptyset))=(P, \emptyset)$, as desired.

Let us remark that the hypothesis of non existence of future sequences with past divergent lifts nor past sequences with future divergent lifts in $M$, even if it appears to be quite strong, it is easily verifiable. In fact, example 5.4 illustrates how to verify the absence of such sequences, while corollary 4.23 gives hypothesis that guarantees such absence.

In any case, whenever $\bar{\pi}$ restricts properly to $\bar{M}$ and it is surjective, we can proceed in complete analogy with the partial cases and obtain the following diagram:

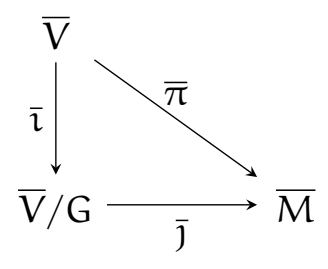

where two points in $\bar{V}$ are G-related if they project by $\bar{\pi}$ into the same point of $\bar{M}$; and $\bar{V} / \mathrm{G}$ denotes the related quotient space. From its definition, $\bar{\jmath}$ defines a bijection between $\bar{V} / \mathrm{G}$ and $\bar{M}$.

As a final remark in this section, simple cases where the map $\bar{\pi}$ is univocally determined are pointed out now.

Lemma 4.7. Assume that the projection $\pi: \mathrm{V} \rightarrow \mathrm{M}$ is tame. If $(\overline{\mathrm{P}}, \emptyset) \sim \mathrm{G}_{0}\left(\overline{\mathrm{P}^{\prime}}, \overline{\mathrm{F}^{\prime}}\right)$, then $\overline{\mathrm{F}^{\prime}}=\emptyset$ (and analogously for the case $(\emptyset, \overline{\mathrm{F}})$ ). In particular, the extended map $\bar{\pi}$ is univocally determined (see remark 4.5). 
Proof. The result is straightforward, once we recall that in tame projections, if $\hat{\pi}(\overline{\mathrm{P}})=$ $\hat{\pi}\left(\overline{P^{\prime}}\right)$, then there exists $g \in G$ such that $\overline{P^{\prime}}=g \bar{P}$. Therefore, if $\overline{F^{\prime}} \neq \emptyset$ and $\overline{P^{\prime}} \sim_{S} \overline{F^{\prime}}$, it follows that $\bar{P}=g^{-1} \overline{P^{\prime}} \sim s g^{-1} \overline{F^{\prime}}$, in contradiction with $\bar{P} \sim_{S} \emptyset$.

Proposition 4.8. Assume that the points $(\mathrm{P}, \mathrm{F}) \in \overline{\mathrm{M}}$ with $\mathrm{P} \neq \emptyset \neq \mathrm{F}$ have lifts in $\overline{\mathrm{V}}$ (see proposition 4.2), $M$ does not admit an inextensible sequence $\left\{x_{n}\right\}_{n} \subset M$ which is either past-directed chronological with future divergent lifts or future-directed chronological with past divergent lifts, and $\bar{M}$ is Hausdorff. Then, $\bar{\pi}$ restricts properly to $\bar{M}$, it is surjective and univocally determined.

Proof. By proposition 4.6 we have that $\bar{\pi}$ restricts properly to $\bar{M}$ and it is surjective, so we only have to show that $\bar{\pi}$ is univocally determined. Having different possible definitions for $\bar{\pi}$ is only possible under the following situation (or its analogous for the future): there exist three points $(\overline{\mathrm{P}}, \emptyset),\left(\overline{\mathrm{P}}_{1}, \overline{\mathrm{F}}_{1}\right),\left(\overline{\mathrm{P}}_{2}, \overline{\mathrm{F}}_{2}\right) \in \overline{\mathrm{V}}$ with $\hat{\pi}(\overline{\mathrm{P}})=\hat{\pi}\left(\overline{\mathrm{P}}_{1}\right)=\hat{\pi}\left(\overline{\mathrm{P}}_{2}\right)=\mathrm{P}$ but with $F_{1}=\check{\pi}\left(\bar{F}_{1}\right) \neq \check{\pi}\left(\overline{\mathrm{F}}_{2}\right)=\mathrm{F}_{2}$. However, from proposition 4.1 we know that both $\left(\mathrm{P}, \mathrm{F}_{1}\right),\left(\mathrm{P}, \mathrm{F}_{2}\right)$ belong to $\overline{\mathrm{M}}$, which is not possible from the Hausdorffness of the latter (observe that any future chronological sequence $\left\{x_{n}\right\}_{n}$ defining $P$ will converge to both points, see theorem 2.8 (ii)).

\subsection{At the chronological level}

Let us now study how is the behaviour of $\bar{\jmath}$ regarding the causal structure. As a first step, we need to define first a chronological relation on $\bar{V} / G$. For this, we will follow an approach inspired from $[20$, section 6.2$]$, where two equivalence classes $\bar{\imath}((\bar{P}, \bar{F})), \bar{\imath}\left(\left(\overline{P^{\prime}}, \overline{F^{\prime}}\right)\right) \in \bar{V} / G$ (being $(\overline{\mathrm{P}}, \overline{\mathrm{F}}),\left(\overline{\mathrm{P}^{\prime}}, \overline{\mathrm{F}^{\prime}}\right) \in \overline{\mathrm{V}}$ two arbitrary points) are chronologically related, $\bar{\imath}((\overline{\mathrm{P}}, \overline{\mathrm{F}})) \ll$ $\bar{\imath}\left(\left(\overline{P^{\prime}}, \overline{F^{\prime}}\right)\right)$ if there exist $\left(\bar{P}_{0}, \bar{F}_{0}\right) \in \bar{\imath}((\bar{P}, \bar{F}))$ and $\left(\overline{P^{\prime}},{\overline{F^{\prime}}}_{0}\right) \in \bar{\imath}\left(\left(\overline{P^{\prime}}, \overline{F^{\prime}}\right)\right)$ with $\left(\bar{P}_{0}, \bar{F}_{0}\right) \ll$ $\left(\overline{\mathrm{P}}_{0}, \overline{\mathrm{F}}_{0}\right)$ in $\overline{\mathrm{V}}$.

In general, and under the hypothesis that $\bar{\pi}$ restricts properly to $\bar{M}$ and it is surjective, we can obtain that both spaces inherits the same causal structure.

Proposition 4.9. Let $\pi: \mathrm{V} \rightarrow \mathrm{M}$ a spacetime covering projection and assume that $\bar{\pi}$ restricts properly to $\overline{\mathrm{M}}$ and it is surjective. Denote by $\bar{\jmath}$ the corresponding map between $\overline{\mathrm{V}} / \mathrm{G}$ and $\overline{\mathrm{M}}$. Then, the bijection $\overline{\mathrm{\jmath}}$ is a chronological isomorphism, that is,

$$
(P, F) \ll\left(P^{\prime}, F^{\prime}\right) \Longleftrightarrow \bar{\jmath}^{-1}((P, F)) \ll \bar{j}^{-1}\left(\left(P^{\prime}, F^{\prime}\right)\right)
$$

Proof. Let us start by fixing some notation. Consider $(\mathrm{P}, \mathrm{F}),\left(\mathrm{P}^{\prime}, \mathrm{F}^{\prime}\right) \in \overline{\mathrm{M}}$ and denote by $(\bar{P}, \bar{F}),\left(\overline{P^{\prime}}, \overline{F^{\prime}}\right) \in \bar{V}$ two corresponding lifts. It follows that $\bar{j}^{-1}((P, F))=\bar{\imath}((\bar{P}, \bar{F}))$ and $\bar{j}^{-1}\left(\left(\mathrm{P}^{\prime}, \mathrm{F}^{\prime}\right)\right)=\bar{\imath}\left(\left(\overline{\mathrm{P}^{\prime}}, \overline{\mathrm{F}^{\prime}}\right)\right)$.

Assume that $\bar{\imath}((\bar{P}, \bar{F})) \ll \bar{\imath}\left(\left(\overline{P^{\prime}}, \overline{F^{\prime}}\right)\right)$ and, without loss of generality, that $(\bar{P}, \bar{F}) \ll$ $\left(\overline{\mathrm{P}^{\prime}}, \overline{\mathrm{F}^{\prime}}\right)$. Then, $\overline{\mathrm{F}} \cap \overline{\mathrm{P}^{\prime}} \neq \emptyset$ and from the first bullet point of proposition 2.9 , that $\mathrm{F} \cap \mathrm{P}^{\prime} \neq \emptyset$. Therefore, $(P, F) \ll\left(P^{\prime}, F^{\prime}\right)$ and the left implication follows.

For the other implication, assume that $(P, F) \ll\left(P^{\prime}, F^{\prime}\right)$, i.e., $F \cap P^{\prime} \neq \emptyset$ and let $x \in F \cap P^{\prime}$. As $x \in F$ and $\check{\pi}(\bar{F})=F$, proposition 2.9 ensures that there exists a point $p \in V$ with $\pi(p)=x$ such that $p \in \bar{F}$. Reasoning in the same way but fixing this lifted $p \in V$ of $x$, we can show that there exists $g \in G$ such that $p \in g \overline{\mathrm{P}^{\prime}}$ (recall that $\left.\hat{\pi}\left(\overline{\mathrm{P}^{\prime}}\right)=\mathrm{P}^{\prime}\right)$. In conclusion, 
$p \in \bar{F} \cap g \overline{P^{\prime}}$ and so $(\bar{P}, \bar{F}) \ll\left(g \overline{P^{\prime}}, g \overline{F^{\prime}}\right)$. Hence, $\bar{\imath}((\bar{P}, \bar{F})) \ll \bar{\imath}\left(\left(\overline{P^{\prime}}, \overline{F^{\prime}}\right)\right)\left(=\bar{\imath}\left(\left(g \overline{P^{\prime}}, g \overline{F^{\prime}}\right)\right)\right)$ and the right implication follows.

\subsection{At the topological level}

Finally, in this section we will compare the topological structures of both, $\bar{V} / \mathrm{G}$ and $\bar{M}$. Let us start by fixing some notation. $\bar{M}$ and $\bar{V}$ will be endowed with the corresponding chronological topology, while $\bar{V} / G$ will be with the induced quotient topology from $\bar{V}$. In concordance with section 3, we will denote by $L_{M}$ the chronological limit on $\bar{M}$, by $L_{V}$ the chronological limit on $\bar{V}$ and by $L_{G}$ the quotient limit operator on $\bar{V} / G$ induced from $L_{V}$ (recall equation (2.2)).

In spite of the partial cases where the openness of the map $\hat{\jmath}$ is always ensured, in general the map $\bar{\jmath}$ is neither continuous nor open. In fact, the following result summarizes the only cases where $\bar{\jmath}$ is well behaved with respect the limit operator.

Proposition 4.10. Let $\pi: \mathrm{V} \rightarrow \mathrm{M}$ be a spacetime covering and assume that $\bar{\pi}$ restricts properly to $\bar{M}$ and it is surjective. Then

(a) If $(\overline{\mathrm{P}}, \overline{\mathrm{F}}) \in \mathrm{L}_{\mathrm{V}}(\bar{\sigma})$ for some sequence $\bar{\sigma} \subset \overline{\mathrm{V}}$ with $\overline{\mathrm{P}} \neq \emptyset \neq \overline{\mathrm{F}}$, then $(\mathrm{P}, \mathrm{F}) \in \mathrm{L}_{\mathrm{M}}(\sigma)$, where $(\mathrm{P}, \mathrm{F})=\bar{\pi}((\overline{\mathrm{P}}, \overline{\mathrm{F}}))$ and $\sigma=\bar{\pi}(\bar{\sigma})$.

(b) If $(\mathrm{P}, \emptyset) \in \mathrm{L}_{M}(\sigma)$ (analogously for $(\emptyset, \mathrm{F}) \in \mathrm{L}_{M}(\sigma)$ ) for some sequence $\sigma \subset \overline{\mathrm{M}}$, then there exist a subsequence $\mathrm{K} \subset \sigma$ and lifts $(\overline{\mathrm{P}}, \emptyset)$ and $\overline{\mathrm{K}}$ of $(\mathrm{P}, \emptyset)$ and $\mathrm{K}$ respectively such that $(\overline{\mathrm{P}}, \emptyset) \in \operatorname{Lv}(\overline{\mathrm{K}})$.

Proof. Assertion (b) is a direct consequence of (2.7), remark 3.5 and the fact that any lift $(\bar{P}, \bar{F}) \in \bar{\pi}^{-1}((P, \emptyset))$ should have $\bar{F}=\emptyset$, so let us focus on assertion (a). For this, recall that from the definition of the chronological limit, $\overline{\mathrm{P}} \subset \operatorname{LI}\left(\left\{\overline{\mathrm{P}}_{n}\right\}_{n}\right)$ and $\overline{\mathrm{F}} \subset \operatorname{LI}\left(\left\{\overline{\mathrm{F}}_{n}\right\}_{n}\right)$. As the projection is well behaved with contentions, we have that $\mathrm{P} \subset \operatorname{LI}\left(\left\{\mathrm{P}_{n}\right\}_{n}\right)$ and $\mathrm{F} \subset \operatorname{LI}\left(\left\{\mathrm{F}_{n}\right\}_{n}\right)$, which is enough to ensure that $(P, F) \in L_{M}\left(\left\{\left(P_{n}, F_{n}\right)\right\}_{n}\right.$ ) (see proposition 2.7).

The other cases (that is, when $(\overline{\mathrm{P}}, \overline{\mathrm{F}})$ has one empty component or when $\mathrm{P} \neq \emptyset \neq \mathrm{F})$ are false in general, as it is proved by examples 5.1 and 5.7. On the first one there exists a sequence $\left\{q_{n}\right\}_{\mathfrak{n}} \subset V$ converging to a point of the form $(\bar{P}, \emptyset)$, while its projection converges to a point $\left(\mathrm{P}^{\prime}, \emptyset\right)$ with $\hat{\pi}(\overline{\mathrm{P}})=\mathrm{P} \subsetneq \mathrm{P}^{\prime}$. On the second example, the sequence $\left\{x_{n}\right\}_{n}$ converges to $(P, F)$ in $\bar{M}$, however $\left\{x_{n}\right\}_{n}$ has no convergent lift on the corresponding $\bar{V}$.

The first case is directly related with the non continuity of $\hat{\jmath}$. In fact, we can easily prove that:

Proposition 4.11. Let $\pi: \mathrm{V} \rightarrow \mathrm{M}$ a spacetime covering with $\bar{\pi}$ restricting properly to $\overline{\mathrm{M}}$ and surjective. If $\bar{\pi}((\overline{\mathrm{P}}, \emptyset))=(\mathrm{P}, \emptyset), \bar{\pi}((\emptyset, \overline{\mathrm{F}}))=(\emptyset, \mathrm{F})$ for any IP $\overline{\mathrm{P}}$ and IF $\overline{\mathrm{F}}$ (so that, in particular, $\bar{\pi}$ is univocally determined, see remark 4.5); and $\mathrm{M}$ has no sequence with divergent lifts, the map $\bar{\pi}$ (and so, $\bar{\jmath}$ ) is continuous.

Proof. For the continuity of $\bar{\pi}$ is enough to show that, given a point $(\overline{\mathrm{P}}, \overline{\mathrm{F}}) \in \overline{\mathrm{V}}$ and a sequence $\left\{\left(\overline{\mathrm{P}}_{n}, \overline{\mathrm{F}}_{n}\right)\right\}_{\mathfrak{n}} \subset \bar{V}$ with $(\overline{\mathrm{P}}, \overline{\mathrm{F}}) \in \operatorname{LV}\left(\left\{\left(\overline{\mathrm{P}}_{n}, \overline{\mathrm{F}}_{\mathfrak{n}}\right)\right\}_{\mathfrak{n}}\right)$, then $(\mathrm{P}, \mathrm{F}) \in \mathrm{L}_{M}\left(\left\{\left(\mathrm{P}_{n}, \mathrm{~F}_{n}\right)\right\}_{\mathfrak{n}}\right)$, where $(P, F)=\bar{\pi}((\bar{P}, \bar{F}))$ and $\left(P_{n}, F_{n}\right)=\bar{\pi}\left(\left(\bar{P}_{n}, \bar{F}_{n}\right)\right)$. If $\bar{P} \neq \emptyset \neq \bar{F}$, the result follows from 
proposition 4.10 (a). If $\bar{F}=\emptyset$ (the other case is analogous) we have that $\bar{P} \in \hat{L}_{V}\left(\left\{\bar{P}_{n}\right\}_{\mathfrak{n}}\right)$ and so, from proposition 3.8, that $\mathrm{P} \in \hat{\mathrm{L}}_{M}\left(\left\{\mathrm{P}_{\mathfrak{n}}\right\}_{\mathfrak{n}}\right)$. Finally, from hypothesis, $\bar{\pi}((\overline{\mathrm{P}}, \emptyset))=$ $(P, \emptyset) \in \bar{M}$, so $(P, \emptyset) \in L_{M}\left(\left\{\left(P_{n}, F_{n}\right)\right\}_{n}\right)$.

Let us give a closer look to previous proof. Observe that the non existence of divergent lifts is used precisely when we deal with limit points of the form $(\bar{P}, \emptyset)$ or $(\emptyset, \bar{F})$. In this case, $\hat{\pi}(\overline{\mathrm{P}}):=\mathrm{P}$ does not belong to $\hat{\mathrm{L}}_{M}\left(\left\{\mathrm{P}_{n}\right\}_{n}\right)$ if it is not a maximal IP in $\operatorname{LS}\left(\left\{\mathrm{P}_{n}\right\}_{n}\right)$ and then, necessarily, it should exists another IP $\mathrm{P}^{\prime}$ with $\mathrm{P} \subsetneq \mathrm{P}^{\prime} \in \hat{\mathrm{L}}_{M}\left(\left\{\mathrm{P}_{n}\right\}_{n}\right)$ (up to a subsequence). Therefore, if we assume that $\bar{M}$ has no lightlike boundary points (and conditions ensuring that $(P, \emptyset) \in \bar{M})$, such a situation is not possible and the continuity of $\bar{\pi}$ follows. In conclusion we have:

Proposition 4.12. Let $\pi: \mathrm{V} \rightarrow \mathrm{M}$ be a projection satisfying: (i) $\bar{\pi}$ restricts properly to $\overline{\mathrm{M}}$ and it is surjective, (ii) $\bar{\pi}((\overline{\mathrm{P}}, \emptyset))=(\mathrm{P}, \emptyset)$ and $\bar{\pi}((\emptyset, \overline{\mathrm{F}}))=(\emptyset, \mathrm{F})$ for any IP $\overline{\mathrm{P}}$ and IF $\overline{\mathrm{F}}$ (hence $\bar{\pi}$ is univocally determined, see remark 4.5); and (iii) $\overline{\mathrm{M}}$ has no lightlike boundary points. Then, the map $\bar{\pi}$ (and so, $\bar{\jmath}$ ) is continuous.

Remark 4.13. Observe that, in spite of corollary 3.9, here we do not need to impose that the boundary $\bar{M}$ has only spatial boundary points; indeed, we can also include timelike ones. The reason is simple: unlike partial boundaries, the total c-completion takes into account more information for each point in the boundary, specially with timelike boundary points where both, the future and past components, are non empty. In fact, such an additional information let us simplify the definition of the limit operator (see proposition 2.7), as we have used on the proof of proposition 4.11.

As we have mention at the beginning of the section, and in spite of the continuity, the openness of the partial maps $\hat{\jmath}$ and $\breve{\jmath}$ is not enough to ensure the openness of $\bar{\jmath}$, as we can see on example 5.7. This means that an additional condition has to be imposed to obtain such an openness. In this sense, we will consider the condition of finite chronology whose properties will be studied in the following section.

\subsection{Group actions with the finite chronology property}

First of all, let us introduce the definition of finite chronology.

Definition 4.14. Let $\mathrm{V}$ be a spacetime and $\mathrm{G}$ a group of isometries. We will say that the pair $(\mathrm{V}, \mathrm{G})$ is finitely chronological if given two points $\mathrm{p}, \mathrm{q} \in \mathrm{V}$ with $\mathrm{p} \ll \mathrm{q}$, there exists only a finite number of elements $\mathrm{g} \in \mathrm{G}$ such that $\mathrm{p} \ll \mathrm{gq}$.

The finite chronology property will be enough to ensure the openness of $\bar{\jmath}$ and it will also simplify the conditions to ensure when the map $\bar{\pi}$ restricts properly to $\bar{M}$, it is univocally determined and surjective. However, such a condition will not be enough to prove the continuity of $\hat{\jmath}$ or $\breve{\jmath}$, as it is showed by example 5.3. Let us begin with a crucial lemma:

Lemma 4.15. Assume that $(\mathrm{V}, \mathrm{G})$ is finitely chronological and consider a point $\mathrm{p} \in \mathrm{V}, a$ past-directed (respectively future-directed) chronological chain $\left\{\mathrm{p}_{\mathrm{n}}\right\}_{\mathrm{n}} \subset \mathrm{V}$ and a sequence $\left\{g_{n}\right\}_{\mathfrak{n}} \subset \mathrm{G}$. If for all $\mathrm{n} \in \mathbb{N}, \mathrm{p} \ll \mathrm{g}_{\mathrm{n}} \mathrm{p}_{\mathrm{n}}$ (respectively $\mathrm{g}_{\mathrm{n}} \mathrm{p}_{\mathrm{n}} \ll \mathrm{p}$ ), then there exists 
$\mathrm{n}_{0} \in \mathbb{N}$ and a finite family $\left\{\mathrm{h}_{1}, \ldots, \mathrm{h}_{\mathrm{r}}\right\} \subset \mathrm{G}$ such that for $\mathrm{n} \geqslant \mathrm{n}_{0}, \mathrm{~g}_{\mathrm{n}}=\mathrm{h}_{\mathrm{i}}$ for some $i=1, \ldots, r$. In fact, $\mathrm{n}_{0}$ can be taken in such a way that each $\mathrm{h}_{\mathrm{i}}$ occurs infinitely often, and so, $\left\{h_{1}, \ldots, h_{r}\right\} \subset G\left(p,\left\{p_{n}\right\}_{n}\right)$ (respectively $\left\{h_{1}, \ldots, h_{r}\right\} \subset G\left(\left\{p_{n}\right\}_{n}, p\right)$, where

$$
\begin{aligned}
G\left(p,\left\{p_{n}\right\}_{n}\right) & :=\left\{g \in G: p \ll g p_{n} \text { for all } n\right\} \\
\left(G\left(\left\{p_{n}\right\}_{n}, p\right):\right. & \left.=\left\{g \in G: g p_{n} \ll p \text { for all } n\right\}\right)
\end{aligned}
$$

is a non empty finite set.

Proof. The proof follows essentially by recalling that, for a fixed $k_{0} \in \mathbb{N}$ and $n \geqslant k_{0}$,

$$
\mathrm{p} \ll g_{\mathrm{n}} \mathrm{p}_{\mathrm{n}} \ll \mathrm{g}_{\mathrm{n}} \mathrm{p}_{\mathrm{k}_{0}} .
$$

In particular, as there exist a finite number of elements $g \in G$ such that $p \ll g p_{k_{0}}, g_{n}$ should belong to a finite family of elements in $\mathrm{G}$ for $\boldsymbol{n}$ big enough. Moreover, we can take $\left\{h_{1} \ldots, h_{r}\right\} \subset G$ such that, for all $h_{i}$, there exists a subsequence $\left\{g_{n_{k}^{i}}\right\}_{k}$ with $g_{n_{k}^{i}}=h_{i}$. In particular, there exists $n_{0}$ such that for each $n \geqslant n_{0}$ there exists $i(\equiv i(n))$ with $g_{n}=h_{i}$.

For the second assertion, recall that the set $G\left(p,\left\{p_{n}\right\}_{\mathfrak{n}}\right)$ is finite by the finitely chronological property. Now, we will show that $\left\{h_{1}, \ldots, h_{r}\right\} \subset G\left(p,\left\{p_{n}\right\}_{n}\right)$. As we have stated before, for each $h_{i}$ there exists a subsequence $\left\{g_{n_{k}^{i}}\right\}_{k} \subset\left\{g_{n}\right\}_{n}$ such that $g_{n_{k}^{i}}=h_{i}$, and therefore satisfying $p \ll g_{n_{k}^{i}} p_{n_{k}^{i}}=h_{i} p_{n_{k}^{i}}$ for all $k$. Now observe that, for any $m \in \mathbb{N}$, we can take $k \in \mathbb{N}$ such that $m<n_{k}^{i}$, and it follows that $p \ll h_{i} p_{n_{k}^{i}} \ll h_{i} p_{m}$ (as $\left\{p_{n}\right\}_{n}$ is past-directed chronological chain); concluding then that $h_{i} \in G\left(p,\left\{p_{n}\right\}_{n}\right)$.

If we consider two points $p, p^{\prime} \in V$ with $p \ll p^{\prime}$, then it follows that $G\left(p^{\prime},\left\{p_{n}\right\}_{n}\right) \subseteq$ $G\left(p,\left\{p_{n}\right\}_{n}\right)$. This relation allow us to prove that the lifts of terminal sets are well behaved, at least when $(\mathrm{V}, \mathrm{G})$ is finitely chronological, with respect to the future and common pasts. Concretely,

Lemma 4.16. Consider an $I P \mathrm{P}$ and an IF $\mathrm{F}$ on $\mathrm{M}$ satisfying that $\mathrm{P} \subset \downarrow \mathrm{F}$; and take $\overline{\mathrm{P}}, \overline{\mathrm{F}}$ the corresponding lifts. If $(\mathrm{V}, \mathrm{G})$ is finitely chronological then the set $\mathrm{G}(\overline{\mathrm{P}}, \overline{\mathrm{F}})$ defined by

$$
\mathrm{G}(\overline{\mathrm{P}}, \overline{\mathrm{F}})=\{\mathrm{g} \in \mathrm{G}: \overline{\mathrm{P}} \subset \downarrow \mathrm{g} \overline{\mathrm{F}}\}
$$

is non empty and finite.

Proof. As a first step, we are going to characterize the set $G(\bar{P}, \bar{F})$ in terms of the sequences defining $\bar{P}$ and $\bar{F}$. In this sense, let $\left\{x_{n}\right\}_{n}$ and $\left\{y_{n}\right\}_{n}$ be chronological sequences defining $P$ and $F$ respectively, and $\left\{p_{n}\right\}_{n},\left\{q_{n}\right\}_{n}$ the corresponding chronological lifts defining $\bar{P}$ and $\overline{\mathrm{F}}$. Observe that the following chain of equivalences follow

$$
\begin{aligned}
g \in G(\bar{P}, \bar{F}) & \Longleftrightarrow \bar{P} \subset \downarrow g \bar{F} \\
& \Longleftrightarrow p_{n} \in g \bar{F} \text { for all } n \in \mathbb{N} \\
& \Longleftrightarrow p_{n} \ll g q_{m} \text { for all } n, m \in \mathbb{N} \\
& \Longleftrightarrow g \in G\left(p_{n},\left\{q_{m}\right\}_{m}\right) \text { for all } n \in \mathbb{N}
\end{aligned}
$$

In particular,

$$
G(\bar{P}, \bar{F})=\cap_{n \in \mathbb{N}} G\left(p_{n},\left\{q_{m}\right\}_{m}\right)
$$


As a second step, recall that from hypothesis $\mathrm{P} \subset \downarrow$ F, and so, $x_{n} \ll y_{m}$ for all $\mathrm{n}, \mathrm{m} \in \mathbb{N}$. Hence, proposition 2.9 ensures that there exists a sequence $\left\{g_{\mathrm{m}}\right\}_{\mathrm{m}} \subset \mathrm{G}$ such that $p_{n} \ll g_{m} q_{m}$ and so, from lemma $4.15, G\left(p_{n},\left\{q_{m}\right\}_{m}\right)$ is non empty and finite for all $n$.

Then, $G(\bar{P}, \bar{F})$ is the intersection of a numerable family of non empty and finite sets ordered by $G\left(p_{n+1},\left\{q_{m}\right\}_{m}\right) \subset G\left(p_{n},\left\{q_{m}\right\}_{m}\right)$. Therefore, it is a non empty and finite set.

In particular, and as a consequence of previous lemma and Props. 4.2 and 4.6, we have that:

Corollary 4.17. Let $\pi: \mathrm{V} \rightarrow \mathrm{M}$ be a spacetime covering with $(\mathrm{V}, \mathrm{G})$ finitely chronological and assume that $M$ does not admit an inextensible sequence $\left\{x_{n}\right\}_{n} \subset M$ which is either past-directed chronological with future divergent lifts or future-directed chronological with past divergent lifts. Then, the map $\bar{\pi}: \bar{V} \rightarrow \bar{M}$ restricts properly to $\bar{M}$ and it is surjective.

At this point a natural question arise at the point set level: is there any relation between $\bar{\pi}^{-1}((P, F))$ and the set $G(\bar{P}, \bar{F})$ ? Intuitively, one can expect that for a fixed lift $\bar{P}$, the set $G(\bar{P}, \bar{F})$ determines all the pairs of the form $(\bar{P}, g \bar{F}) \in \bar{V}$ with projection $(P, F)$. However, as we recall in remark 4.3, it is not clear that, in general, all the lifts preserving the relation with the common future (or past) are S-related. Again, the finite chronology condition will be enough for this, as we will see on lemma 4.20. In order to prove such a lemma, we need first the following technical result:

Lemma 4.18. Let $\overline{\mathrm{P}}, \overline{\mathrm{P}^{\prime}} \in \hat{\mathrm{V}}$ (respectively $\overline{\mathrm{F}}, \overline{\mathrm{F}^{\prime}} \in \check{\mathrm{V}}$ ) be two points of the future (past) causal completion projecting to the same set $\mathrm{P} \in \hat{\mathrm{M}}(\mathrm{F} \in \check{M})$. Suppose one of the following situations:

(H1) $(\mathrm{V}, \mathrm{G})$ is finitely chronological and there exists $\mathrm{p} \in \mathrm{V}$ such that $\overline{\mathrm{P}^{\prime}} \subset \mathrm{I}^{-}(\mathrm{p}) \overline{\mathrm{F}^{\prime}} \subset$ $\left.\mathrm{I}^{+}(\mathrm{p})\right)$.

(H2) G is finite.

Then there exists $\mathrm{h}^{\prime} \in \mathrm{G}$ such that $\overline{\mathrm{P}}=\mathrm{h}^{\prime} \overline{\mathrm{P}^{\prime}}\left(\overline{\mathrm{F}}=\mathrm{h}^{\prime} \overline{\mathrm{F}^{\prime}}\right)$. In particular, it follows that if $\mathrm{G}$ is finite the projection $\pi$ is future (past) tame.

Proof. Let $\left\{p_{n}\right\}_{n},\left\{p_{n}^{\prime}\right\}_{n}$ be future chronological chains defining $\bar{P}$ and $\overline{P^{\prime}}$ respectively. As both sets project onto the same $\mathrm{P}$, it follows that the projection of such sequences $\left\{x_{n}\right\}_{n},\left\{x_{n}^{\prime}\right\}_{n}$ generate $P$. In particular, for each $n$ there exists $m(n)$ big enough such that $x_{n} \ll x_{m(n)}^{\prime}$. We will consider $\{m(n)\}_{n}$ a strictly increasing sequence, so $\left\{x_{m(n)}^{\prime}\right\}_{\mathfrak{n}}$ is a subsequence of $\left\{x_{n}^{\prime}\right\}_{\mathfrak{n}}$ and generates the same $P$ (and, accordingly, $\left\{p_{m(n)}^{\prime}\right\}_{n}$ generates $\left.\overline{\mathrm{P}^{\prime}}\right)$. From proposition 2.9 it follows that there exists a sequence $\left\{g_{\mathfrak{n}}\right\}_{\mathfrak{n}} \subset \mathrm{G}$ such that $g_{n} p_{n} \ll p_{m(n)}^{\prime}$ for all $n$.

Now observe that, in either situation (H1) nor (H2), and up to a subsequence, $\left\{g_{n}\right\}_{n}$ can be considered a constant sequence ( say $g_{n}=h \in G$ for all $n$ ). In the case that $G$ is finite the argument is straightforward. In the other case, recall that from (H1) we have that $g_{\mathfrak{n}} p_{\mathfrak{n}} \ll p_{m(n)}^{\prime} \ll p$, and so the assertion follows from lemma 4.15. Therefore, 
$h p_{n} \ll p_{m(n)}^{\prime}$ for all $n$, and hence, $h \bar{P} \subset \overline{P^{\prime}}$. By interchanging the roles of $\overline{P^{\prime}}$ and $h \bar{P}$ (recall that, now, $h \overline{\mathrm{P}} \subset \overline{\mathrm{P}^{\prime}} \subset \mathrm{I}^{-}(\mathrm{p})$ ), we find another $\tilde{h}$ such that $\tilde{h} \overline{\mathrm{P}^{\prime}} \subset \mathrm{h} \overline{\mathrm{P}}$ or, by considering $h^{\prime}=h^{-1} \tilde{h}$, that $h^{\prime} \overline{P^{\prime}} \subset \bar{P}$.

Now, we can join both contentions $h \bar{P} \subset \overline{P^{\prime}}$ and $h^{\prime} \overline{P^{\prime}} \subset \bar{P}$ together in the following way

$$
g \overline{\mathrm{P}} \subset \mathrm{h}^{\prime} \overline{\mathrm{P}^{\prime}} \subset \overline{\mathrm{P}}
$$

for $g=h^{\prime} h$; and then construct the chain:

$$
\overline{\mathrm{P}} \supset \mathrm{g} \overline{\mathrm{P}} \supset(\mathrm{g})^{2} \overline{\mathrm{P}} \supset \cdots \supset(\mathrm{g})^{\mathrm{n}} \overline{\mathrm{P}} \supset \ldots
$$

where $(g)^{i}$ denotes the iteration of the action by $g$ i-times. Now observe that under the hypothesis of the lemma, there exists $i_{0}$ such that $(g)^{i_{0}}=e$. This assertion is again straightforward under the assumption of $\mathrm{G}$ finite, so let us focus on the hypothesis (H1). If by contradiction $(g)^{i} \neq(g)^{j}$ for all $i \neq j$, and recalling that $\overline{\mathrm{P}} \subset \mathrm{I}^{-}(\mathrm{p})$, we deduce that $(g)^{i} \bar{P} \subset I^{-}(p)$ for all $i$. which contradicts that $(V, G)$ is finitely chronological (the point $p$ will be chronologically related with $(g)^{i} q$ for any $q \in \bar{P}$ and $\left.i \in \mathbb{N}\right)$.

Summarizing we deduce that $g \bar{P}=\bar{P}$ and from (4.6) we obtain that $\bar{P}=h^{\prime} \overline{P^{\prime}}$, as desired.

Remark 4.19. Observe that we have also proved in previous lemma that if $g \bar{P} \subset \bar{P}$ for some $\mathrm{g}$ and, or $\mathrm{G}$ is finite, or $(\mathrm{V}, \mathrm{G})$ is finitely chronological and there exists $p \in V$ with $\overline{\mathrm{P}} \subset \mathrm{I}^{-}(\mathrm{p})$, then $\mathrm{g} \overline{\mathrm{P}}=\overline{\mathrm{P}}$ (an analogous result for past sets follows).

Lemma 4.20. Assume that $(\mathrm{V}, \mathrm{G})$ is finitely chronological. If $\overline{\mathrm{P}}$ and $\overline{\mathrm{F}}$ are terminal sets with $\hat{\pi}(\overline{\mathrm{P}})=\mathrm{P} \sim_{\mathrm{S}} \mathrm{F}=\check{\pi}(\overline{\mathrm{F}})$, then $\overline{\mathrm{P}} \sim_{\mathrm{s}} \mathrm{g} \overline{\mathrm{F}}$ for all $\mathrm{g} \in \mathrm{G}(\overline{\mathrm{P}}, \overline{\mathrm{F}})$.

Proof. Assume without loss of generality that $e \in G(\bar{P}, \bar{F})$, and so, that $\bar{P} \subset \downarrow \bar{F}$. By contradiction, let us assume that $\bar{P}$ is not S-related with $\bar{F}$. Recalling remark 4.3, we ensure the existence of a terminal set $\overline{\mathrm{P}^{\prime}}$ with $\overline{\mathrm{P}} \subsetneq \overline{\mathrm{P}^{\prime}} \subset \downarrow \overline{\mathrm{F}}$ and satisfying that $\hat{\pi}(\overline{\mathrm{P}})=\hat{\pi}\left(\overline{\mathrm{P}^{\prime}}\right)$. As $(\mathrm{V}, \mathrm{G})$ is finite chronological and there exists $\mathrm{p} \in \mathrm{V}$ such that $\overline{\mathrm{P}^{\prime}}, \overline{\mathrm{P}} \subset \mathrm{I}^{-}(\mathrm{p})$ (take any $p \in \bar{F})$, lemma 4.18 ensures that there exists $h \in G$ such that $\overline{P^{\prime}}=h \bar{P}$. But then, recalling remark 4.19, we arrive to a contradiction with $\overline{\mathrm{P}} \subsetneq \overline{\mathrm{P}^{\prime}}=\mathrm{h} \overline{\mathrm{P}}$.

The technical lemma 4.18 allow us to prove that $\bar{\pi}$ is univocally determined, as it follows from the following result (recall also remark 4.5):

Proposition 4.21. Assume that $(\mathrm{V}, \mathrm{G})$ is finitely chronological. If $(\overline{\mathrm{P}}, \emptyset) \sim \mathrm{G}_{0}\left(\overline{\mathrm{P}^{\prime}}, \overline{\mathrm{F}^{\prime}}\right)$, then $\overline{\mathrm{F}^{\prime}}=\emptyset$ (an analogous result follow for a pair $(\emptyset, \overline{\mathrm{F}})$ ).

Proof. Assume by contradiction that $(\overline{\mathrm{P}}, \emptyset) \sim_{\mathrm{G}_{0}}\left(\overline{\mathrm{P}^{\prime}}, \overline{\mathrm{F}^{\prime}}\right)$ with $\overline{\mathrm{F}^{\prime}} \neq \emptyset$. By recalling that both sets $\bar{P}$ and $\overline{P^{\prime}}$ projects onto the same set $\mathrm{P}$ in $\hat{M}$, and that for any $p \in \overline{F^{\prime}}, \overline{P^{\prime}} \subset I^{-}(p)$; we can apply lemma 4.18 (H1) deduce the existence of $h^{\prime} \in G$ such that $\bar{P}=h^{\prime} \overline{P^{\prime}}$. Since $\mathrm{G}$ acts by isometries, it follows that $\overline{\mathrm{P}}=\mathrm{h}^{\prime} \overline{\mathrm{P}^{\prime}} \sim_{S} \mathrm{~h}^{\prime} \overline{\mathrm{F}^{\prime}}$, which is a contradiction to $\overline{\mathrm{P}} \sim_{\mathrm{S}} \emptyset$. In conclusion, $\overline{\mathrm{F}^{\prime}}=\emptyset$.

With all previous machinery set, we are now in conditions to prove the openness of $\bar{\jmath}$ under the assumption of finite chronology: 
Proposition 4.22. Let $\pi: \mathrm{V} \rightarrow \mathrm{M}$ be spacetime covering projection with $(\mathrm{V}, \mathrm{G})$ finitely chronological and assume that $\bar{\pi}$ restricts properly to $\bar{M}$ and it is surjective. Then, the univocally determined map $\bar{\pi}$ induces an open map $\bar{\jmath}$ from $\bar{V} / \mathrm{G}$ to $\overline{\mathrm{M}}$.

Proof. Let $\left\{\left(\mathrm{P}_{n}, \mathrm{~F}_{n}\right)\right\}_{n} \subset \bar{M}$ be a sequence and $(\mathrm{P}, \mathrm{F}) \in \overline{\mathrm{M}}$ a point such that $(\mathrm{P}, \mathrm{F}) \in$ $L_{M}\left(\left\{\left(P_{n}, F_{n}\right)\right\}_{n}\right)$. Our aim is to show that, up to a subsequence, $\left(P_{n}, F_{n}\right)$ and $(P, F)$ admit $\operatorname{lifts}\left({\overline{\mathrm{P}^{\prime}}}_{n}, \overline{\mathrm{F}^{\prime}} \bar{n}^{\prime}\right)$ and $\left(\overline{\mathrm{P}^{\prime}}, \overline{\mathrm{F}^{\prime}}\right)$ with $\left(\overline{\mathrm{P}^{\prime}}, \overline{\mathrm{F}^{\prime}}\right) \in \operatorname{Lv}\left(\left\{\left(\overline{\mathrm{P}}_{n},{\overline{\mathrm{F}^{\prime}}}_{n}\right)\right\}_{n}\right)$, and hence, that $\bar{j}^{-1}((\mathrm{P}, \mathrm{F})) \in$ $\mathrm{L}_{\mathrm{G}}\left(\left\{\bar{j}^{-1}\left(\mathrm{P}_{\mathfrak{n}}, \mathrm{F}_{\mathrm{n}}\right)\right\}_{\mathfrak{n}}\right)$ (recall $\left.(2.2)\right)$. Observe that the case where $\mathrm{F}$ or $\mathrm{P}$ is empty follows from proposition 4.10 (b), so we only need to focus on the case where both sets are non empty.

Assume that $P \neq \emptyset \neq F$ and let $\bar{P}, \bar{F}, \bar{P}_{n}, \bar{F}_{n}$ be some fixed lifts of $P, F, P_{n}, F_{n}$ respectively. Consider $\left\{x_{n}\right\}_{n}$ and $\left\{y_{n}\right\}_{n}$ chronological sequences defining $P$ and $F$ and, as usual, denote by $\left\{p_{n}\right\}_{n}$ and $\left\{q_{n}\right\}_{n}$ the corresponding lifts defining $\bar{P}$ and $\bar{F}$. Let us denote by $\{m(n)\}_{\mathfrak{n}}$ a sequence in $\mathbb{N}$ with $m(n+1) \geqslant m(n)+1$ and satisfying that $x_{n} \in P_{m(n)}$ and $y_{n} \in F_{m(n)}$. Now, as $x_{n} \in P_{m(n)}$, proposition 2.9 ensures that $p_{n} \in g_{n} \bar{P}_{m(n)}$ for some $g_{n} \in G$. From lemma 4.16, we know that the set $G\left(g_{n} \bar{P}_{m(n)}, \bar{F}_{m(n)}\right)$ is non empty and, from lemma 4.20, that for any $g_{n}^{\prime} \in G\left(g_{n} \bar{P}_{m(n)}, \bar{F}_{m(n)}\right), g_{n} \bar{P}_{m(n)} \sim s g_{n}^{\prime} \bar{F}_{m(n)}$. Finally, again from proposition 2.9 and $y_{n} \in F_{m(n)}$, there exists $h_{n} \in G$ such that $h_{n} q_{n} \in g_{n}^{\prime} \bar{F}_{m(n)}$.

Now, let us observe that from $g_{n} \bar{P}_{m(n)} \subset \downarrow g_{n}^{\prime} \bar{F}_{m(n)}$, it follows that $p_{n} \ll h_{n} q_{n}$. In particular, we have the chain

$$
p_{1} \ll p_{n} \ll h_{n} q_{n}
$$

and then, from lemma 4.15, we can ensure that, up to a subsequence, $\left\{h_{\mathfrak{n}}\right\}_{\mathfrak{n}}$ is constant, say $h_{n}=h \in G$ for all $n$. In particular, for any $i$ and all $n>i$, it follows that

$$
p_{i} \ll p_{n} \ll h q_{n} .
$$

In particular, $\bar{P} \subset \downarrow h \bar{F}$ and so $h \in G(\bar{P}, \bar{F})$. Hence, lemma 4.20 ensures that both sets $\overline{\mathrm{P}}$ and $h \overline{\mathrm{F}}$ are S-related.

Summarizing:

- The pairs $(\overline{\mathrm{P}}, \mathrm{h} \overline{\mathrm{F}})$ and $\left(g_{\mathfrak{n}} \overline{\mathrm{P}}_{\mathfrak{m}(\mathfrak{n})}, g_{\mathfrak{n}}^{\prime} \overline{\mathrm{F}}_{\mathfrak{m}(\mathfrak{n})}\right)$ belongs to $\overline{\mathrm{V}}$.

- $\overline{\mathrm{P}} \subset \operatorname{LI}\left(\left\{g_{\mathfrak{n}} \overline{\mathrm{P}}_{\mathbf{m}(\mathfrak{n})}\right\}_{\mathfrak{n}}\right)$ and $h \overline{\mathrm{F}} \subset \operatorname{LI}\left(\left\{g_{\mathfrak{n}}^{\prime} \overline{\mathrm{F}}_{\mathfrak{m}(\mathfrak{n})}\right\}_{\mathfrak{n}}\right)$, thus (see proposition 2.7)

$$
(\bar{P}, h \bar{F}) \in \operatorname{Lv}\left(\left\{\left(g_{n} \bar{P}_{m(n)}, g_{n}^{\prime} \bar{F}_{m(n)}\right)\right\}_{n}\right) .
$$

In conclusion, and always up to a subsequence, if $(P, F) \in L_{M}\left(\left\{\left(P_{n}, F_{n}\right)\right\}_{n}\right)$ we can always obtain appropriate lifts $\left(\overline{\mathrm{P}^{\prime}}, \overline{\mathrm{F}^{\prime}}\right)$ and $\left\{\left(\overline{\mathrm{P}^{\prime}}, \overline{\mathrm{F}^{\prime}}{ }_{n}\right)\right\}_{n}$ such that $\left(\overline{\mathrm{P}^{\prime}}, \overline{\mathrm{F}^{\prime}}\right) \in$ $\operatorname{LV}\left(\left\{\left(\overline{\mathrm{P}^{\prime}} n, \overline{\mathrm{F}^{\prime}} n\right)\right\}_{n}\right)$. The result follows then as a consequence of proposition 2.3 applied to $\bar{\jmath}^{-1}$.

As a final remark of this section, we will show how finite chronology let us simplify some of our previous hypothesis for the definition and continuity of $\bar{\jmath}$. In fact, the condition of $M$ having no sequence with divergent lifts (which is almost equivalent to the continuity of $\hat{\pi}$ and $\check{\pi}$, recall proposition 3.8) imposed in proposition 4.6 can be substituted by a topological requirement on $\hat{V}$ and $\check{V}$ respectively: 
Corollary 4.23. Assume that $(\mathrm{V}, \mathrm{G})$ is finitely chronological and that both $\hat{\mathrm{V}}, \check{\mathrm{V}}$ are Hausdorff. Then, $\bar{\pi}$ restricts properly to $\bar{M}$, it is surjective and univocally determined.

Proof. We only need to show, according to corollary 4.17, that any past-directed chronological chain on $M$ has no future divergent lifts (the other case will be completely analogous). Let $\left\{y_{n}\right\}_{n}$ be a past-directed chronological chain and consider $\left\{q_{n}\right\}_{n}$ a past chronological sequence in $V$ with $\pi\left(q_{n}\right)=y_{n}$ and defining a IF $\bar{F}$. Suppose that there exist $\left\{h_{n}\right\}_{n},\left\{g_{n}\right\}_{n} \subset$ $\mathrm{G}$ and $\overline{\mathrm{P}}, \overline{\mathrm{P}^{\prime}} \in \hat{\mathrm{V}}$ such that $\overline{\mathrm{P}} \in \hat{\mathrm{L}}_{V}\left(\left\{\mathrm{I}^{-}\left(\mathrm{h}_{n} \mathbf{q}_{n}\right)\right\}_{n}\right)$ and $\overline{\mathrm{P}^{\prime}} \in \hat{\mathrm{L}}_{V}\left(\left\{\mathrm{I}^{-}\left(\mathrm{g}_{n} \mathbf{q}_{\mathrm{n}}\right)\right\}_{n}\right)$.

Take $p \in \bar{P}$. From $\bar{P} \in \hat{L}_{V}\left(\left\{I^{-}\left(h_{n} q_{n}\right)\right\}_{n}\right)$ we have that $p \ll h_{n} q_{n}$ for $n$ big enough. As $(V, G)$ is finitely chronological, lemma 4.15 ensures that, up to a subsequence, $h_{n}=h_{0}$ for some fixed $h_{0} \in G$. Reasoning in the same way with $\overline{P^{\prime}}$ and $\left\{g_{\mathfrak{n}}\right\}_{\mathfrak{n}}$, we can ensure that, up to subsequence, $g_{n}=g_{0}$ for some fixed $g_{0} \in G$.

Hence, we have that $\overline{\mathrm{P}} \in \hat{\mathrm{L}}_{V}\left(\left\{\mathrm{I}^{-}\left(\mathrm{h}_{\mathrm{o}} \mathbf{q}_{\mathfrak{n}}\right)\right\}_{\mathfrak{n}}\right)$ and $\overline{\mathrm{P}^{\prime}} \in \hat{\mathrm{L}}_{V}\left(\left\{\mathrm{I}^{-}\left(\mathrm{g}_{\mathrm{o}} \mathbf{q}_{\mathfrak{n}}\right)\right\}_{\mathfrak{n}}\right)$ and, from the first inclusion, we deduce that $\left(g_{0} h_{0}^{-1}\right) \bar{P} \in \hat{L}_{V}\left(\left\{I^{-}\left(g_{0} q_{n}\right)\right\}_{n}\right)$. As $\hat{V}$ is Hausdorff, then $\left(g_{0} h_{0}^{-1}\right) \bar{P}=\overline{P^{\prime}}$ and both sets projects into the same set in $\hat{M}$. In conclusion, $\left\{y_{n}\right\}_{n}$ cannot admit future divergent lists. Finally, $\bar{\pi}$ is univocally determined as it follows from proposition 4.21 .

At the topological level, we also have to impose some conditions on $\bar{M}$, obtaining:

Corollary 4.24. Assume that $(\mathrm{V}, \mathrm{G})$ is finitely chronological, $\hat{\mathrm{V}}$ and $\check{\mathrm{V}}$ are Hausdorff and $\bar{M}$ has no lightlike boundary points. Then, $\bar{V} / \mathrm{G} \equiv \overline{\mathrm{M}}$, i.e., both $\overline{\mathrm{V}} / \mathrm{G}$ and $\overline{\mathrm{M}}$ are homeomorphic and chronologically isomorphic.

Proof. From corollary 4.23 follows that $\bar{\pi}$ restricts properly to $\bar{M}$, it is surjective and univocally determined. Then, Props. 4.9 and 4.22 ensure both, that $\bar{\jmath}$ is a chronological isomorphism and an open map.

Hence, it only rest to show that $\bar{\jmath}$ is continuous. But this follows from proposition 4.12, recalling that proposition 4.21 ensures that $\bar{\pi}((\bar{P}, \emptyset))=(P, \emptyset)$ and $\bar{\pi}((\emptyset, \bar{F}))=(\emptyset, F)$.

Ideally, one would like to impose conditions only on $\bar{V}$ in order to ensure that $\bar{V} / \mathrm{G}$ and $\bar{M}$ have the same structures. For example, and in the spirit of corollary 4.24, we would like to impose on $\bar{V}$ the non-existence of lightlike boundary points to obtain the non-existence of lightlike boundary points on $\bar{M}$, and therefore the continuity of $\bar{\jmath}$. However, the lack of lightlike boundary points in $\bar{V}$ is not enough to ensure the same property on $\bar{M}$ (see example 5.8). Nevertheless the situation is very controlled and it is related again with the existence of very particular divergent lifts. In fact, we can prove that (compare with proposition 3.7):

Corollary 4.25. Let $\pi: \mathrm{V} \rightarrow \mathrm{M}$ be a spacetime projection. Assume that $\bar{\pi}$ restricts properly to $\bar{M}$, it is surjective and it satisfies that $\bar{\pi}((\overline{\mathrm{P}}, \emptyset))=(\mathrm{P}, \emptyset)$ and $\bar{\pi}((\emptyset, \overline{\mathrm{F}}))=(\emptyset, \mathrm{F})$ (hence univocally determined, see remark 4.5) for any IP $\overline{\mathrm{P}}$ and IF $\overline{\mathrm{F}}$. If $\overline{\mathrm{V}}$ has no lightlike boundary points and the G-orbits for both $\hat{\mathrm{V}}$ and $\check{\mathrm{V}}$ are closed (with the corresponding topologies), then $\bar{M}$ has no lightlike boundary points. 
Proof. Assume by contradiction that $\bar{M}$ has lightlike boundary points, that is, that there exists $(\mathrm{P}, \emptyset) \in \bar{M}$ and $\mathrm{P}^{\prime} \in \hat{\mathrm{M}}$ such that $\mathrm{P} \subsetneq \mathrm{P}^{\prime}$ (the case with past sets will be analogous). Let $\left\{x_{n}\right\}_{n}$ and $\left\{x_{n}^{\prime}\right\}_{n}$ be chronological chains generating $P$ and $P^{\prime}$ respectively and consider $\bar{P}, \overline{P^{\prime}},\left\{p_{n}\right\}_{n}$ and $\left\{p_{n}^{\prime}\right\}_{n}$ the corresponding lifts on $\hat{V}$. From hypothesis, it follows that $(\overline{\mathrm{P}}, \emptyset) \in \overline{\mathrm{V}}$.

As $\mathrm{P} \subset \mathrm{P}^{\prime}$ we deduce that, for all $\mathrm{n}, \mathrm{x}_{\mathrm{n}} \ll x_{n^{\prime}}^{\prime}$ with $\mathrm{n}^{\prime}$ big enough, so proposition 2.9 ensures that there exists $g_{n}$ such that $p_{n} \ll g_{n} p_{n}^{\prime} \in g_{n} \overline{P^{\prime}}$. It follows then that $\bar{P} \subset$ $\operatorname{LI}\left(\left\{g_{n} \overline{\mathrm{P}^{\prime}}\right\}_{n}\right)$. Moreover, it also follows that $\overline{\mathrm{P}} \in \hat{\mathrm{L}}_{V}\left(\left\{g_{n} \overline{\mathrm{P}^{\prime}}\right\}_{n}\right)$ as, otherwise, there exists $\overline{\mathrm{P}^{\prime \prime}}$ such that $\overline{\mathrm{P}} \subsetneq \overline{\mathrm{P}^{\prime \prime}}$ and this is not possible as $\overline{\mathrm{V}}$ has no lightlike boundary points.

Finally, and from the hypothesis that the G-orbits are closed on $\hat{V}$ with the future chronological topology, it follows that $\overline{\mathrm{P}} \in\left\{g \overline{\mathrm{P}}^{\prime}\right\}_{\mathbf{g} \in \mathrm{G}}$, i.e., there exists $g_{0} \in \mathrm{G}$ such that $\overline{\mathrm{P}}=$ $g_{0} \overline{\mathrm{P}^{\prime}}$. In conclusion, and taking projections, we obtain that $\mathrm{P}=\mathrm{P}^{\prime}$, a contradiction.

As a consequence of corollaries $4.23,4.24$ and 4.25 , we obtain the following result:

Corollary 4.26. Assume that $(\mathrm{V}, \mathrm{G})$ is finitely chronological, $\overline{\mathrm{V}}$ has no lightlike boundary points and $\hat{\mathrm{V}}$ and $\check{\mathrm{V}}$ are Hausdorff and have closed $\mathrm{G}$-orbits. Then, $\overline{\mathrm{V}} / \mathrm{G} \equiv \overline{\mathrm{M}}$, i.e., both $\overline{\mathrm{V}} / \mathrm{G}$ and $\overline{\mathrm{M}}$ are homeomorphic and chronologically isomorphic.

\subsection{Proof of theorem 1.2}

At the point set level, the first assertion on (PS1) is proved on proposition 4.6 and the second one follows from proposition 4.2 and lemma 4.16. (PS2) is a consequence of remark 4.5, lemma 4.7 and proposition 4.21; while (PS3) is proved in corollary 4.23.

At the chronological level, assertion $(\mathrm{CH})$ is proved on proposition 4.9.

At the topological level, (TP1) (i) is proved in proposition 4.11, (TP1) (ii) is proposition 4.12. The conditions over the projection of pairs $(\overline{\mathrm{P}}, \emptyset)$ and $(\emptyset, \overline{\mathrm{F}})$ remarked in (TP1) are proved when $\pi$ is tame or $(\mathrm{V}, \mathrm{G})$ finitely chronological by lemma 4.7 or by proposition 4.21 respectively. Finally, (TP2) follows from proposition 4.22.

For the last assertions, $\bar{\pi}$ being univocally determined is a consequence of the finite chronology and (PS2). (a) follows from (PS1), (CH), (TP1) (i) and (TP2), while for (b) we have to consider (PS3) and (TP1) (ii) instead of (PS1) and (TP1) (i). The last assertion (c) is proved in corollary 4.26, recalling that the closedness of the G-orbits under $\pi$ tame is proved in proposition 3.7 .

\section{On the optimality of the results: some examples}

Along this section, we will include some examples showing that our main results are optimal. It is worth pointing out that in all the examples $\# \mathrm{~L}_{M}(\sigma)$ will be bounded, and so, according to lemma 2.4 , that $\mathrm{L}_{M}$ will be of first order UTS. This is specially relevant recalling proposition 3.8 , as it means that in all our examples the non existence of divergent lifts characterize the continuity of $\hat{\pi}$ and $\check{\pi}$.

Let us start with the example due to Harris where $\hat{\jmath}$ is not continuous. Here, we will include only the main properties of his example, referring the reader to [19] for details. 


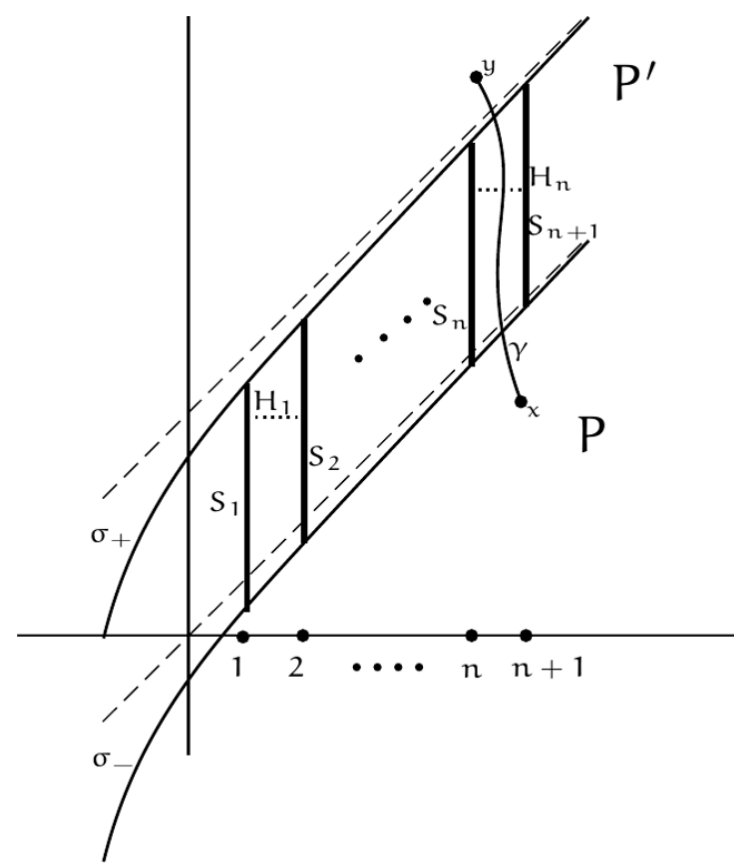

(A)

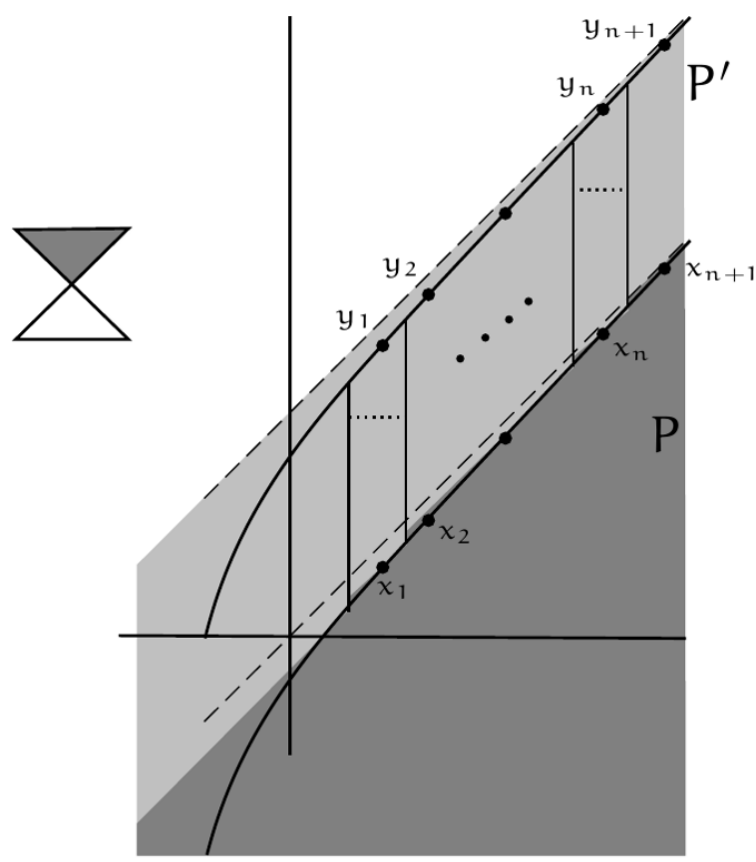

(B)

Figure 1. The space $M$ is constructed in the following way: consider in $\mathbb{L}^{2}$ two timelike curves $\sigma_{-}$ and $\sigma_{+}$approaching two parallel lightlike lines, as we can see in (A). $M$ is obtained by removing from $\mathbb{L}^{2}$ the segments $S_{\mathfrak{n}}$ obtained by joining vertically the points $\sigma_{-}(\mathfrak{n})$ and $\sigma_{+}(\mathfrak{n})$. The universal cover $V$ of $M$ contains then a numerable family of copies of $M$ glued along the segments $H_{i}$ coherently (see details on example 5.1 and [19]).

Example 5.1. (Behaviour of the universal cover and non-continuity of $\hat{\pi}$ ) In this example we will see: first, the main properties about the universal cover of a spacetime $M$ where we have removed a numerable family of compact segments (these properties will be used frequently on the forthcoming examples). Second, a case where $\hat{\pi}$ (and so, $\hat{\jmath}$ ) is noncontinuous. Finally, that $\hat{\mathrm{V}} / \hat{\mathrm{G}}$ is not a $\mathrm{T}_{1}$-topological space.

Let us consider a spacetime $\mathrm{M}$ as in the figure 1, and let $\mathrm{V}$ denote its universal cover. As it is described in the last example of [19], $\mathrm{V}$ contains a numerable family of copies of $M$, that we will denote by $\{\mathrm{n}\} \times M$ with $\mathrm{n} \in \mathbb{Z}$, glued coherently along the segments $\mathrm{H}_{n}$. For a given element $\mathrm{x} \in \mathrm{M}$, let us denote by $\mathrm{p}$ its lift in $\mathrm{V}$ living in the fibre $\{0\} \times M$. We will also denote by $\mathrm{n} \cdot \mathrm{p}$ the lift of $\mathrm{x}$ in the fibre $\{\mathrm{n}\} \times M$ (i.e., $\mathrm{p} \equiv \mathbf{0} \cdot \mathrm{p}$ ).

In order to understand how the fibres are glued along $\mathrm{H}_{\mathrm{n}}$, let us show how the lifts of curves behave. Consider $\gamma$ a curve on $\mathrm{M}$ as it is showed in figure 1 (A), which is a timelike curve joining two points $\mathrm{x}$ and $\mathrm{y}$. Let $\mathrm{p}$ and $\mathrm{q}$ be the corresponding lifts in the fibre $\{0\} \times \mathrm{M}$ and consider $\bar{\gamma}$ a lift of $\gamma$ on $\mathrm{V}$ with start point $\mathrm{m} \cdot \mathrm{p}$. The fibres are glued in such a way that, as $\gamma$ intersects the segment $\mathrm{H}_{n}$, the lifted curve $\bar{\gamma}$ moves from the fibre $\{\mathrm{m}\} \times M$ to $\{\mathrm{m}+\mathrm{n}\} \times \mathrm{M}$, being $(\mathrm{m}+\mathrm{n}) \cdot \mathrm{q}$ its final point.

Once we have pointed out this behaviour, let us observe the particularities of the example regarding the continuity of $\hat{\pi}$. Let us observe now figure 1 (B), where we have two TIPs 
$\mathrm{P} \subsetneq \mathrm{P}^{\prime}$ defined by the sequences $\left\{\mathrm{x}_{\mathrm{n}}\right\}_{\mathrm{n}}$ and $\left\{\mathrm{y}_{\mathrm{n}}\right\}_{\mathrm{n}}$ ( $\mathrm{P}$ is filled in dark grey, while $\mathrm{P}^{\prime}$ has a lighter grey). Consider $\mathrm{p}_{\mathrm{n}}$ and $\mathbf{q}_{\mathrm{n}}$ lifts of $\mathrm{x}_{\mathrm{n}}$ and $\mathrm{y}_{\mathrm{n}}$ respectively living in the fibre $\{0\} \times \mathrm{M}$. It is not difficult to observe, due to the behaviour described before, that $\mathrm{m} \cdot \mathrm{p}_{\mathrm{n}} \nless \mathrm{m} \cdot \mathrm{q}_{\mathrm{n}}$ for any $\mathrm{m} \in \mathbb{N}$. In fact, it follows that

$$
\mathrm{m} \cdot \mathrm{p}_{\mathrm{n}} \ll(\mathrm{m}+\mathrm{n}) \cdot \mathrm{q}_{\mathrm{n}} \quad \text { for all } \mathrm{n} \in \mathbb{N}
$$

as we can consider timelike curves on $M$ joining $x_{n}$ with $y_{n}$ and intersecting $\mathrm{H}_{n}$. From this, we can prove that: (a) the sequence $\bar{\sigma}=\left\{\mathrm{I}^{-}\left(\mathrm{q}_{\mathrm{n}}\right)\right\}_{\mathrm{n}}$ has $\overline{\mathrm{P}^{\prime}}$ (the lift of $\mathrm{P}^{\prime}$ on the fibre $\{0\} \times M)$ on its limit, (b) the sequence $\left\{\mathrm{I}^{-}\left(\mathrm{n} \cdot \mathrm{q}_{\mathrm{n}}\right)\right\}_{\mathrm{n}}$ has $\overline{\mathrm{P}}$ on its limit (the inclusion on the inferior limit is straightforward, while the proof of the maximal character is detailed in [19]) and $(c) \hat{\pi}(\overline{\mathrm{P}})=\mathrm{P} \subsetneq \mathrm{P}^{\prime}=\hat{\pi}\left(\overline{\mathrm{P}^{\prime}}\right)$. In conclusion, and recalling proposition 3.8, $\hat{\pi}$ is not continuous.

As a final observation, let us consider the future causal completion of the universal cover $\mathrm{V}$ (which is a $\mathrm{T}_{1}$ topological space) and its quotient space $\hat{\mathrm{V}} / \hat{\mathrm{G}}$, where $\mathrm{G}=\pi_{1}(\mathrm{M})$ is the fundamental group of $\mathrm{M}$ acting on $\mathrm{V}$. Consider $\overline{\mathrm{P}^{\prime}} \in \hat{\mathrm{V}}$ and its corresponding class $\left[\overline{\mathrm{P}^{\prime}}\right]$ in the quotient space $\hat{\mathrm{V}} / \hat{\mathrm{G}}$. It is now straightforward to see that $\overline{\mathrm{P}^{\prime}} \in \hat{\mathrm{L}}_{\mathrm{V}}\left(\left\{\overline{\mathrm{P}^{\prime}}\right\}_{\mathfrak{n}}\right)$ and $\overline{\mathrm{P}} \in \hat{\mathrm{L}}_{\mathrm{V}}\left(\left\{\mathrm{n} \cdot \overline{\mathrm{P}^{\prime}}\right\}_{\mathfrak{n}}\right)$. Hence, recalling the definition of $\mathrm{L}_{\mathrm{Q}}$ (see $\left.(2.2)\right)$, it follows that both $[\overline{\mathrm{P}}]$ and $\left[\overline{\mathrm{P}^{\prime}}\right]$ belong to $\mathrm{L}_{\mathrm{Q}}\left(\left\{\left[\overline{\mathrm{P}^{\prime}}\right]\right\}_{\mathfrak{n}}\right)$, making $\hat{\mathrm{V}} / \hat{\mathrm{G}}$ a non $\mathrm{T}_{1}$ topological space.

Example 5.2. (Optimality of proposition 4.6) The following example shows that a point $(\overline{\mathrm{P}}, \emptyset) \in \overline{\mathrm{V}}$ (respectively $(\emptyset, \overline{\mathrm{F}})$ ) is not well projected (recall proposition 4.1), even when $\hat{\pi}$ and $\check{\pi}$ are continuous. With this aim, a point $(\mathrm{P}, \mathrm{F}) \in \overline{\mathrm{M}}$ with no natural lift on $\overline{\mathrm{V}}$ will be exhibited.

Let us consider $\mathrm{M}$ a spacetime as described in figure 2 and $\mathrm{V}$ its universal cover. As it is pointed out in [5, figure 11], both sets $\mathrm{P} \sim_{\mathrm{S}} \mathrm{F}$ are S-related. Now, let us fix $\overline{\mathrm{P}}$ and $\overline{\mathrm{F}}$ lifts of the corresponding terminal sets on $\{0\} \times \mathrm{M} \subset \mathrm{V}$ as we have done on example 5.1; and denote by $\left\{\mathrm{p}_{\mathrm{n}}\right\}_{\mathrm{n}} \subset\{0\} \times M$ a future chronological sequence which is lift of the sequence $\left\{x_{n}\right\}_{n}$ showed in figure 2. Recall that the lifts on $\mathrm{V}$ of timelike curves of $\mathrm{M}$ moving between $S_{n}$ and $S_{n+1}$ behave essentially as described in example 5.1. Hence, it follows that

$$
\cap_{n \in \mathbb{N}} I^{+}\left(p_{n}\right)=\emptyset \text {. }
$$

In fact, for a given point $\mathrm{y} \in \mathrm{F}$ (and so, with $\mathrm{x}_{\mathrm{n}} \ll \mathrm{y}$ for all $\mathrm{n}$ ) with fixed lift $\mathrm{q} \in\{0\} \times \mathrm{M} \subset$ $\mathrm{V}$, there is no constant element $\mathrm{g} \in \mathrm{G}$ such that $\left(\mathrm{p}_{1} \ll\right) \mathrm{p}_{\mathrm{n}} \ll \mathrm{gq}$ for all $\mathrm{n}$ big enough (since $\mathrm{p}_{\mathrm{n}} \ll \mathrm{mq}_{\mathrm{n}}$ for $\left.\mathrm{m} \geqslant \mathrm{n}\right)$. This shows in particular that $(\mathrm{V}, \mathrm{G})$ is not finitely chronological (hence theorem 1.2 (PS3) is not applicable) and that the set $\uparrow \overline{\mathrm{P}}$ is empty, so $\overline{\mathrm{P}} \sim_{\mathrm{S}} \emptyset$.

However, it is not difficult to see that both $\hat{\pi}$ and $\check{\pi}$ are continuous. Recall that the non-continuity of such maps can only follow by the existence of a sequence $\left\{y_{n}\right\}_{n} \subset M$ admitting divergent lifts.

The only case we have to be concerned is when $\left\{y_{n}\right\}_{n}$ converges on $\mathbb{R}^{2}$, or to the point $(0,1)$ or to $(0,0)$ (in the other cases, the convergence is essentially the usual one in $\mathbb{R}^{2}$ ). Assume for instance that the sequence $\left\{\mathrm{y}_{n}\right\}_{n}$ converges to the point $(0,1)$ (the other case is completely analogous). It is straightforward to check that any convergent lift with the past chronological topology of $\left\{\mathrm{y}_{\mathrm{n}}\right\}_{\mathrm{n}}$ in $\mathrm{V}$ are, up to a subsequence, of the form $\left\{\mathrm{m} \cdot \mathrm{q}_{\mathrm{n}}\right\}_{\mathrm{n}}$, with 


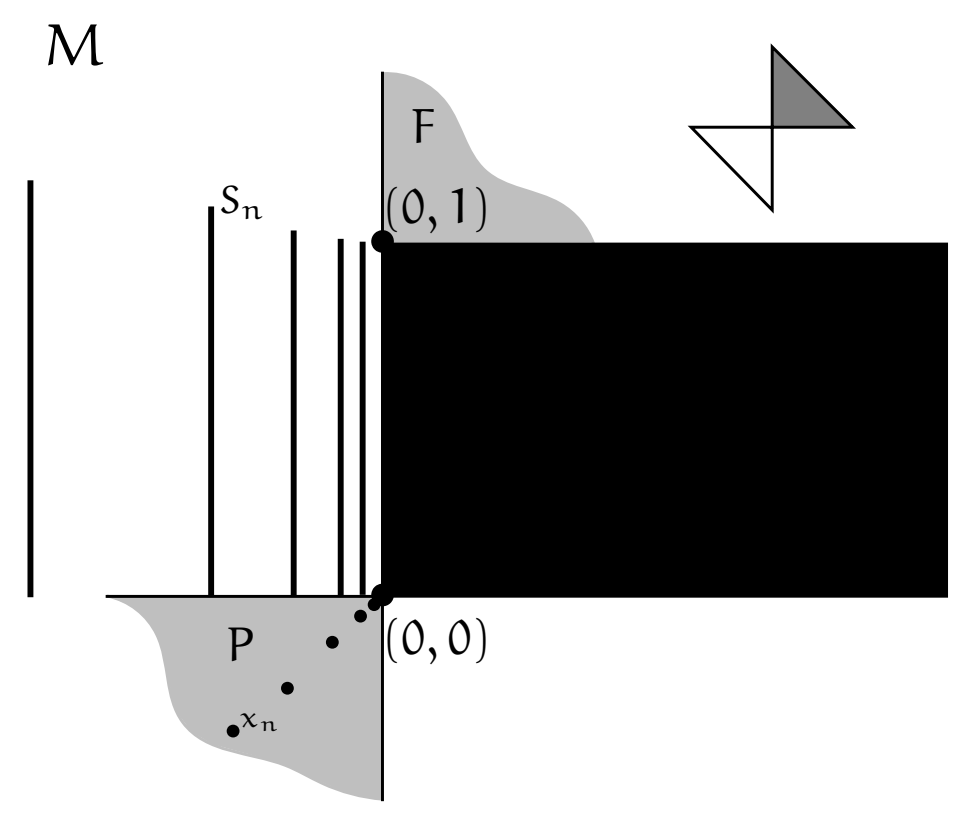

Figure 2. $M$ is constructed by removing from $\mathbb{L}^{2}$ the black square and the vertical segments $S_{n}$. As it was pointed out in [5, figure 11], the terminal sets $\mathrm{P}$ and $\mathrm{F}$ are $\mathrm{S}$-related, and so, they form a pair $(P, F) \in \bar{M}$. However, if $\bar{P}$ is a lift of $P$ to the universal cover $V$, it follows that $\uparrow \bar{P}=\emptyset$.

$\mathrm{m} \in \mathbb{Z}$ constant and $\mathrm{q}_{\mathrm{n}} \in\{0\} \times \mathrm{M} \subset \mathrm{V}$ a fixed lift of $\left\{\mathrm{y}_{\mathrm{n}}\right\}_{\mathrm{n}}$. In particular, their limits are of the form $\mathrm{m} \cdot \overline{\mathrm{F}}$. This is due the fact that the IFs involved will not have points between the segments $\mathrm{S}_{\mathrm{n}}$, and so, we do not have to move between different fibres of $\mathrm{V}$. Therefore, any convergent lift of $\left\{\mathrm{y}_{\mathrm{n}}\right\}_{\mathrm{n}}$ with the past topology converges to a terminal set on $\check{\pi}^{-1}(\mathrm{~F})$, and so, $\left\{\mathrm{y}_{\mathrm{n}}\right\}_{\mathrm{n}}$ does not have past divergent lifts (condition (ii) in definition 3.6 cannot be fulfilled).

For the future topology however the situation is a little more technical, as the involved IPs contain these points between segments $\mathrm{S}_{\mathrm{n}}$. With some effort, it can be proved that if $\operatorname{LI}\left(\left\{\mathrm{I}^{-}\left(\mathrm{g}_{\mathrm{n}} \mathrm{q}_{\mathrm{n}}\right)\right\}_{\mathrm{n}}\right) \neq \emptyset$ for some $\left\{\mathrm{g}_{\mathrm{n}}\right\}_{\mathrm{n}} \subset \mathbb{Z}$, then $\mathrm{LI}\left(\left\{\mathrm{I}^{-}\left(\mathrm{g}_{\mathrm{n}} \mathrm{q}_{\mathrm{n}}\right)\right\}_{\mathrm{n}}\right)=\mathrm{m} \overline{\mathrm{P}}$ for some $\mathrm{m} \in \mathbb{Z}$. In particular, any convergent lift with the future topology of $\left\{\mathrm{y}_{\mathrm{n}}\right\}_{\mathrm{n}}$ will converge to some TIP on $\hat{\pi}^{-1}(\mathrm{P})$, and so, reasoning as in previous case, $\left\{\mathrm{y}_{\mathrm{n}}\right\}_{\mathrm{n}}$ does not admits future divergent lifts.

In conclusion, $\mathrm{M}$ does not admit (future or past) divergent lifts, and so, both $\hat{\pi}$ and $\check{\pi}$ are continuous.

Example 5.3. (Optimality of several previous results) Let us see: (i) sequences with divergent lifts can be obtained by a constant sequence $\left\{g_{n}\right\}_{n}$ (see definition 3.6 and theorem 3.8), (ii) if a past chronological sequence has future divergent lifts, then the thesis of proposition 4.1 can fail and (iii) the finite chronology property is not enough to ensure the continuity of the partial maps $\hat{\pi}$ and $\check{\pi}$, being necessary to include them additionally. 


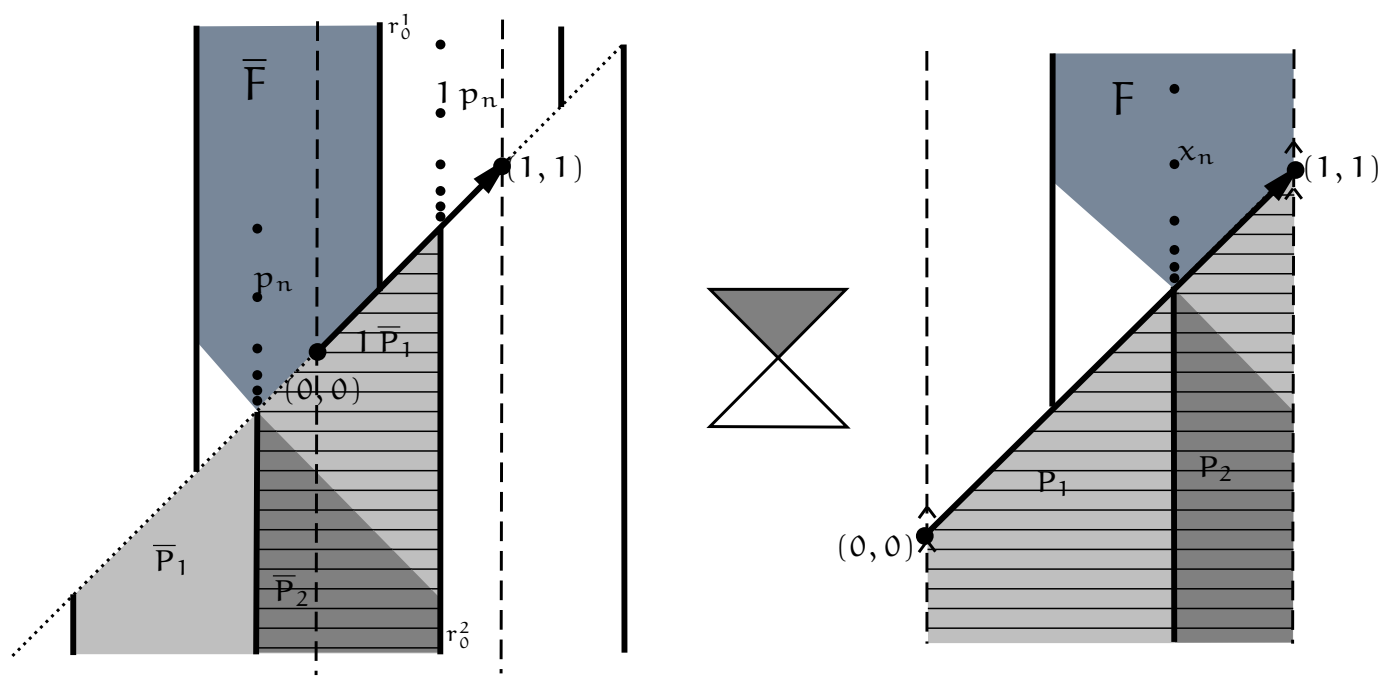

Figure 3. The space $V$ (on the left) is $\mathbb{L}^{2}$ with two families of lines $\left\{r_{n}^{1}\right\}_{n}$ and $\left\{r_{n}^{2}\right\}_{n}$ removed, where $r_{n}^{1}=\{(1 / 3+n, t): t \geqslant 1 / 3+n\}$ and $r_{n}^{2}=\{(2 / 3+n, t): t \leqslant 2 / 3+n\}$. The action of an element $g$ of the group $\mathbb{Z}$ is just a translation of $g$-times the vector $(1,1)$, being the region between the striped lines in the left figure a fundamental region for the action. The quotient space $M=V / \mathbb{Z}$ (on the right) is the space $(0,1) \times \mathbb{R}$ with the points $(0, t)$ and $(1, t+1)$ identified.

Let us consider a space $\mathrm{V} \subset \mathbb{R}^{2}$ as showed in figure 3. On such a space, consider $\mathrm{G} \equiv \mathbb{Z}$ an isometry group given by the following action:

$$
\begin{aligned}
\mathbb{Z} \times V & \rightarrow V \\
(g, p) & \rightarrow g \cdot p:=p+g(1,1) .
\end{aligned}
$$

The quotient $\mathrm{M}=\mathrm{V} / \mathbb{Z}$ can be seen as a cylinder with some cuts on it (see figure $3(B)$ ). Let us summarize the properties of the spacetime covering projection $\pi: \mathrm{V} \rightarrow \mathrm{M}$. On the one hand, and observing figure $3(B)$, it follows easily that $\bar{M}$ contains the pairs $\left(\mathrm{P}_{1}, \mathrm{~F}\right)$ and $\left(\mathrm{P}_{2}, \emptyset\right)$. Indeed, both sets $\mathrm{P}_{1}, \mathrm{P}_{2}$ are contained in $\downarrow \mathrm{F}$, but thanks to the identification of both lateral sides, it follows that $\mathrm{P}_{2} \subsetneq \mathrm{P}_{1}$, so only $\mathrm{P}_{1}$ is maximal on the common past of $\mathrm{F}$. However, on $\overline{\mathrm{V}}$ we have both pairs $\left(\overline{\mathrm{P}}_{1}, \overline{\mathrm{F}}\right)$ and $\left(\overline{\mathrm{P}}_{2}, \overline{\mathrm{F}}\right)$, so the thesis on proposition 4.1 is false on this case.

On the other hand, the non continuity of $\hat{\pi}$ can be deduced from the fact that $\overline{\mathrm{P}}_{2} \in$ $\hat{\mathrm{L}}_{\mathrm{V}}\left(\left\{\mathrm{p}_{\mathrm{n}}\right\}_{\mathrm{n}}\right)$ while $\mathrm{P}_{2}=\hat{\pi}\left(\mathrm{P}_{2}\right) \notin \hat{\mathrm{L}}_{\mathrm{M}}\left(\left\{x_{\mathrm{n}}\right\}_{\mathrm{n}}\right)$, as $\mathrm{P}_{1}$ breaks the maximality of $\mathrm{P}_{2}$ in the superior limit. Finally, it is quite straightforward to see that $(\mathrm{V}, \mathrm{G})$ is finitely chronological. If $\mathrm{p} \ll \mathrm{q}$ in $\mathrm{V}$, it could exists (at most) one element in $\mathrm{g} \in \mathbb{Z}$ such that $\mathrm{p} \ll \mathrm{g} \mathrm{q}$ (specifically, $\mathrm{g}= \pm 1)$. However, we cannot apply theorem 1.2 to ensure that $\bar{\pi}$ restricts properly to $\bar{M}$ as $\hat{\mathrm{V}}$ is not Hausdorff (recall that $\overline{\mathrm{P}}_{1}, \overline{\mathrm{P}}_{2} \in \hat{\mathrm{L}}_{\mathrm{V}}\left(\left\{\mathrm{p}_{\mathrm{n}}\right\}_{\mathrm{n}}\right)$ ).

Example 5.4. ( $\bar{\pi}$ restricts properly even when $\pi$ is non tame) Let us consider the spacetime $\mathrm{V}=\mathbb{R} \times(-1,1) \times \mathbb{R}$ with the 3-dimensional Minkowski metric

$$
\mathfrak{g}=d x^{2}+d y^{2}-d t^{2} .
$$



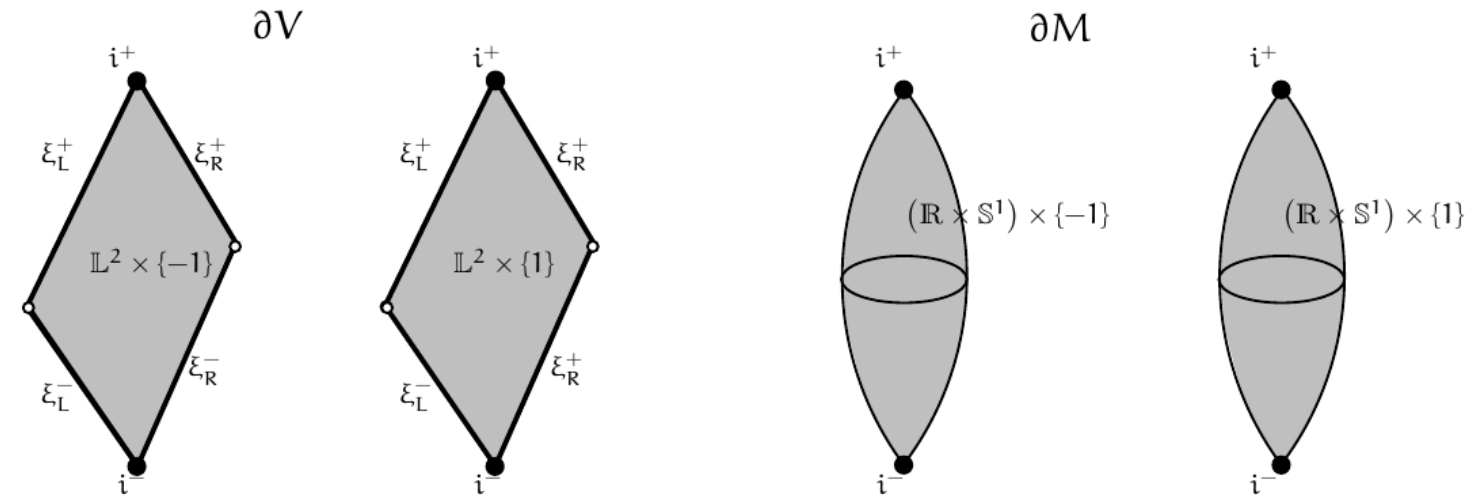

Figure 4. On the left figure is represented the c-boundary of $\mathrm{V}$, which is formed by two copies of the c-completion of the 2-dimensional Minkowski spacetime glued by the corresponding edges (the identified edges are denoted equally). On the right figure it is represented the c-boundary of $\partial \mathrm{M}$, formed by two copies of $\mathbb{R} \times \mathbb{S}^{1}$ and the points $i^{+}$and $i^{-}$(again identified).

As the spacetime $(\mathrm{V}, \mathrm{g})$ is, in fact, a static spacetime, we can calculate directly its cboundary which structure is given by:

$$
\partial V=\left(\left(\xi_{R}^{+} \cup \xi_{L}^{+}\right) \sqcup\left(\xi_{R}^{-} \cup \xi_{L}^{-}\right)\right) \cup\left(\mathbb{L}^{2} \times\{-1,1\}\right) \cup\left\{i^{+}, i^{-}\right\}
$$

(see figure 4). Concretely, recall that the structure of the c-boundary for static models depends essentially on the so-called Busemann completion of its spatial fibre (see for instance [17, theorem 3.10] as well as section 6 for details). In this case, it follows that the associated Busemann completion for $\left(\mathbb{R} \times(-1,1), \mathrm{d}^{2}+\mathrm{dy}^{2}\right)$ is formed by the Cauchy boundary $(\mathbb{R} \times\{-1\}) \cup(\mathbb{R} \times\{1\})$ and two additional points, each determined by inextensible curves whose $\mathrm{x}$-component diverge (one point when the $\mathrm{x}$-component diverges to $+\infty$ and the other to $-\infty)$. The Cauchy boundary points generate in the c-boundary two copies of $\mathbb{L}^{2}$ (denoted in figure 4 by $\mathbb{L}^{2} \times\{-1\}$ and $\mathbb{L}^{2} \times\{1\}$ ) formed by timelike points, and so, with both components non empty; while the two points in the proper Busemann boundary generates four lightlike lines, two for the future and two for the past boundary, denoted by $\xi_{\mathrm{R}}^{+}, \xi_{\mathrm{L}}^{+}, \xi_{\mathrm{R}}^{-}$and $\xi_{\mathrm{L}}^{-}$respectively From the topological viewpoint, and due the simplicity of the example, it follows that the chronological topology works as expected in this c-completion, being the convergence with the chronological topology the same as the usual convergence in figure 4 (after the appropriate identifications).

In previous space we define the following group action

$$
\begin{aligned}
\mathbb{Z} \times V & \rightarrow \mathrm{V} \\
(z,(x, y, t)) & \rightarrow(x+z, y, t)
\end{aligned}
$$

so $\mathrm{M}=\mathrm{V} / \mathbb{Z}$ is, in fact, $\mathrm{M}=\mathbb{S}^{1} \times(-1,1) \times \mathbb{R}$ with the induced metric. Again, the c-boundary is computable by previous methods, obtaining

$$
\partial M=\left(\left(\mathbb{R} \times \mathbb{S}^{1}\right) \times\{-1,1\}\right) \cup\left\{\mathfrak{i}^{+}, \mathfrak{i}^{-}\right\}
$$


(here, the proper Busemann boundary is empty while the Cauchy boundary is formed by two copies of $\left.\mathbb{R} \times \mathbb{S}^{1}\right)$.

Let us describe briefly how $\bar{\pi}$ works: it takes all the lightlike points of $\hat{\partial} \mathrm{V}$ and $\partial \mathrm{V}$ to $\mathrm{i}^{+}$ and $\mathfrak{i}^{-}$respectively; and it mods out by a properly discontinuous $\mathbb{Z}$-action on each $\mathbb{L}^{2} \times\{ \pm 1\}$. In particular, observe that any boundary point in $\left(\xi_{\mathbb{R}}^{+} \backslash\left\{i^{+}\right\}\right) \subset \partial \vee$ and $i^{+} \in \partial \mathrm{V}$ are both projected to $i^{+} \in \partial M$, but no element on $\mathbb{Z}$ send an element in the former to the latter (this can be seen as no translation in $\mathbb{L}^{2}$ sends a terminal set $\overline{\mathrm{P}} \in \xi_{\mathrm{R}}^{+} \backslash\left\{i^{+}\right\}$to $i^{+}$); proving that $\hat{\pi}$ is not (future) tame. In order to show that proposition 4.6 is applicable, we have to show that any inextensible past chronological sequence on $\mathrm{M}$ has no future divergent lifts (being the future case completely analogous).

Let $\sigma=\left\{\left(x_{n}, y_{n}, t_{n}\right)\right\}_{n}$ be a past inextensible chronological sequence on $M$, and let $\bar{\sigma}=$ $\left\{\left(x_{n}+z_{n}, y_{n}, t_{n}\right)\right\}_{n}$ an $\overline{\sigma^{\prime}}=\left\{\left(x_{n}+z_{n}+z_{n}^{\prime}, y_{n}, t_{n}\right)\right\}_{n}$ be two lifts on $\mathrm{V}$. The inextensibility of $\sigma$ determines two possibilities: or $\left\{\mathrm{t}_{n}\right\}_{n} \searrow-\infty$, or $\left\{\mathrm{t}_{n}\right\}_{n} \searrow \Omega$ and $\left\{\mathrm{y}_{n}\right\}_{n}$ converges to some point in $\{-1,1\}$. Observe that in the first case there is nothing to do as $\hat{\mathrm{L}}_{\mathrm{chr}}(\bar{\sigma})=$ $\hat{\mathrm{L}}_{\mathrm{chr}}\left(\overline{\sigma^{\prime}}\right)=\emptyset$. Hence we can assume that we are in the second case and, without loss of generality, that $\left\{y_{n}\right\}_{n} \rightarrow 1$. Recalling the (well) behaviour of the topology in the future completion, if $\hat{\mathrm{L}}(\bar{\sigma}) \neq \emptyset \neq \hat{\mathrm{L}}\left(\overline{\sigma^{\prime}}\right)$ then $\left\{\left(\mathrm{x}_{\mathrm{n}}+z_{\mathrm{n}}, \mathrm{y}_{\mathrm{n}}, \mathrm{t}_{\mathrm{n}}\right)\right\}_{\mathrm{n}} \rightarrow\left(\mathrm{x}_{0}, 1, \Omega\right)$ and $\left\{\left(\mathrm{x}_{\mathrm{n}}+z_{\mathrm{n}}+\right.\right.$ $\left.\left.z_{n}^{\prime}, y_{n}, t_{n}\right)\right\}_{n} \rightarrow\left(x_{0}^{\prime}, 1, \Omega\right)$ for some $x_{0}, x_{0}^{\prime} \in \mathbb{R}$. In particular, and given that $\left\{x_{n}+z_{n}\right\}_{n} \rightarrow$ $x_{0},\left\{x_{n}+z_{n}+z_{n}^{\prime}\right\}_{n} \rightarrow x_{0}^{\prime}$ and $z_{n}^{\prime} \in \mathbb{Z}$, we conclude that for $n$ big enough $z_{n}^{\prime}=z_{0}^{\prime}$ for some fixed $z_{0}^{\prime}$. It follows then that $x_{0}^{\prime}=x_{0}+z_{0}^{\prime}$ and, therefore, that if $\bar{\sigma}$ and $\overline{\sigma^{\prime}}$ have both limit points, such limits points are unique and project into the same point in $\hat{\mathrm{M}}$. In conclusion, the sequence $\sigma$ has no future divergent lifts.

Example 5.5. (Optimality of several results) In this example we will show: (i) a case of a non tame spacetime covering projection, where (and unlike example 5.4) two terminal sets $\overline{\mathrm{P}}, \overline{\mathrm{P}^{\prime}} \in \hat{\mathrm{V}}$ project into the same set on $\hat{\mathrm{M}}$ with no element in the group $\mathrm{G}$ sending one to the other but with $\overline{\mathrm{P}^{\prime}} \mathrm{S}$-related to a non empty set, (ii) that even if $\mathrm{M}$ does not admit constant sequences with future divergent lifts, the G-orbits can be non closed and (iii) a spacetime covering projection with a sequence $\left\{\mathrm{q}_{\mathrm{n}}\right\}_{\mathrm{n}} \subset \mathrm{V}$ and a TIP $\overline{\mathrm{P}} \in \hat{\mathrm{V}}$ with $\overline{\mathrm{P}} \sim_{\mathrm{S}} \emptyset$ and such that $\overline{\mathrm{P}} \in \hat{\mathrm{L}}_{\mathrm{V}}\left(\left\{\mathrm{I}^{-}\left(\mathbf{q}_{\mathrm{n}}\right)\right\}_{\mathrm{n}}\right), \mathrm{P} \in \hat{\mathrm{L}}_{\mathrm{M}}\left(\left\{\mathrm{I}^{-}\left(\mathrm{y}_{\mathrm{n}}\right)\right\}_{\mathrm{n}}\right)$ but $\mathrm{P} \sim_{\mathrm{S}} \mathrm{F}$ with $\mathrm{F} \neq \emptyset$ (showing the optimality of proposition 4.11).

Let us consider the Lorentz manifold

$$
M=\mathbb{L}^{2} \backslash\left(\{[0, \infty) \times 0\} \cup \cup_{n}\left\{\left(0,-\frac{1}{n}\right)\right\}\right)
$$

(see figure 5), and take $\mathrm{V}$ its universal cover. The behaviour of the lifts of curves in $\mathrm{M}$ to $\mathrm{V}$ behaves essentially in the same manner described in example 5.1, that is, it contains a numerable family of copies of $\mathrm{M}$ (which will be denoted again by $\{\mathrm{n}\} \times \mathrm{M}$ ) glued together accordingly; and whenever a curve $\gamma \subset M$ pass between two holes of $M$, the initial point and the endpoint of the lifted curve $\bar{\gamma}$ live in two different fibres of such a numerable family.

It follows that the point $(0,0) \in \mathbb{R}^{2}$ has associated in $\bar{M}$ a singular point $(\mathrm{P}, \mathrm{F}) \in \overline{\mathrm{M}}$. However, the lift of the terminal set $\mathrm{P}$ in a concrete fibre, say $\{0\} \times \mathrm{M}$, determines two different terminal sets $\overline{\mathrm{P}}, \overline{\mathrm{P}^{\prime}}$. The reason is simple, any timelike curve joining a point of 

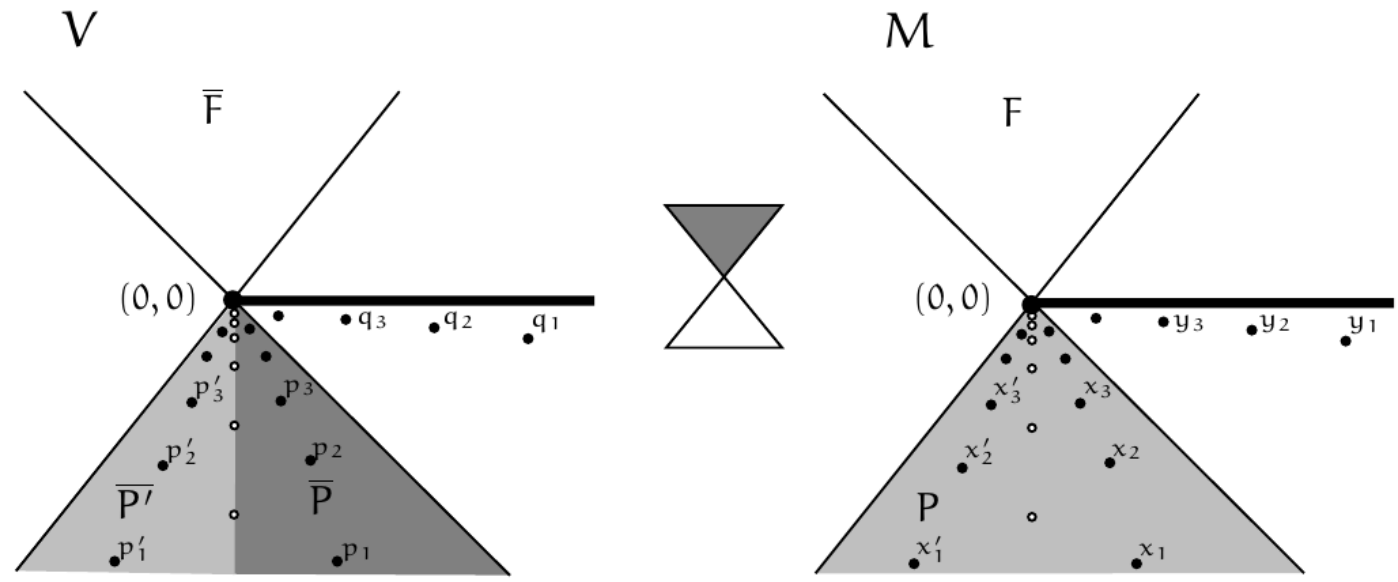

Figure 5. The space $M$ (on the right) is $\mathbb{L}^{2}$ with the line $r=\{(x, 0): x \geqslant 0\}$ and the sequence of points $\left\{\left(0,-\frac{1}{n}\right)\right\}_{n}$ removed, while $V$ is the universal cover of $M$. On the first one, associated to the point $(0,0)$ we have the point $(P, F) \in \partial M$. However, the set $P$ lifts to a fixed fibre $\{0\} \times M \subset V$ as two different terminal past sets $\bar{P}$ and $\overline{P^{\prime}}$, creating two different points $\left(\overline{P^{\prime}}, \bar{F}\right),(\bar{P}, \emptyset) \in \partial V$. In particular, it follows that the sequence $\left\{y_{n}\right\}_{n}$ depicted on the right is not convergent, while its lifts $\left\{\boldsymbol{q}_{\mathfrak{n}}\right\}_{\mathfrak{n}}$ converges to $(\overline{\mathrm{P}}, \emptyset)$.

the sequence $\left\{x_{n}\right\}_{n}$ with $\left\{x_{n}^{\prime}\right\}_{n}$ should pass between two holes of $M$, and so, its lift moves along different fibres. Moreover, from construction, we have that for each $\mathrm{p}_{\mathrm{n}}$ there exists $g_{n}$ ensuring that $p_{n} \in g_{n} \overline{P^{\prime}}$. However, the sequence $\left\{g_{n}\right\}_{n}$ cannot be considered constant (not even up to a subsequence), so there is no $\mathrm{g} \in \mathrm{G}$ such that $\overline{\mathrm{P}} \subset \mathrm{g} \overline{\mathrm{P}^{\prime}}$ and the projection cannot be tame. Moreover, it follows from the construction that $\overline{\mathrm{P}} \subset \operatorname{LI}\left(\left\{\mathrm{g}_{\mathfrak{n}} \overline{\mathrm{P}^{\prime}}\right\}_{\mathfrak{n}}\right)$ and it is maximal on the superior limit, i.e., $\overline{\mathrm{P}} \in \hat{\mathrm{L}} \mathrm{V}\left(\left\{\mathrm{g}_{\mathrm{n}} \overline{\mathrm{P}^{\prime}}\right\}_{\mathfrak{n}}\right)$. Therefore, the $\mathrm{G}$-orbit $\left\{\mathrm{g} \overline{\mathrm{P}^{\prime}}\right\}_{\mathrm{g} \in \mathrm{G}}$ is not closed as $\overline{\mathrm{P}}$ is an element not belonging to the $\mathrm{G}$-orbit of $\overline{\mathrm{P}^{\prime}}$ but which is in its closure.

Let us now show the existence of a sequence $\left\{\mathbf{q}_{\mathrm{n}}\right\}_{\mathrm{n}}$ as described in the first paragraph of the example. Consider a sequence $\left\{\mathrm{y}_{\mathrm{n}}\right\}_{\mathfrak{n}}$ as in figure 5 and $\left\{\mathbf{q}_{\mathrm{n}}\right\}_{\mathfrak{n}}$ its lift in the fibre $\{0\} \times M \subset \mathrm{V}$. As we can see in the figure, $\mathrm{P} \in \hat{\mathrm{L}}_{\mathrm{M}}\left(\left\{\mathrm{I}^{-}\left(\mathrm{y}_{\mathrm{n}}\right)\right\}_{\mathrm{n}}\right)$ and $\overline{\mathrm{P}} \in \hat{\mathrm{L}}_{\mathrm{V}}\left(\left\{\mathrm{I}^{-}\left(\mathrm{q}_{\mathrm{n}}\right)\right\}_{\mathrm{n}}\right)$. Moreover, as we have mention before, $\mathrm{P} \sim_{\mathrm{S}} \mathrm{F}$ with $\mathrm{F} \neq \emptyset$. So, it only rest to show that $\overline{\mathrm{P}} \sim_{\mathrm{S}} \emptyset$. But this follows from the fact that $\uparrow \overline{\mathrm{P}}=\emptyset$ (recall that whenever a timelike curve moves through the space between two holes, it pass to another fibre in $\mathrm{V})$. Summarizing, we have shown in particular that the map $\bar{\pi}$ is not continuous. The sequence $\left\{\mathbf{q}_{\mathrm{n}}\right\}_{\mathrm{n}}$ converges to the point $(\overline{\mathrm{P}}, \emptyset) \in \overline{\mathrm{V}}$, while its projection $\left\{\mathrm{y}_{\mathfrak{n}}\right\}_{\mathfrak{n}}$ does not converge to $(\mathrm{P}, \mathrm{F}) \in \overline{\mathrm{M}}$ (note that $\left.\operatorname{LI}\left(\left\{\mathrm{I}^{+}\left(\mathrm{y}_{\mathrm{n}}\right)\right\}_{\mathrm{n}}\right)=\emptyset\right)$.

Example 5.6. (Several candidates for the projection of a pair) The following example is a three dimensional version of the previous one, and aims to show three points $(\overline{\mathrm{P}}, \emptyset),\left(\overline{\mathrm{P}^{\prime}}, \overline{\mathrm{F}}_{1}\right),\left(\overline{\mathrm{P}^{\prime}}, \overline{\mathrm{F}}_{2}\right) \in \overline{\mathrm{V}}$ with $\hat{\pi}(\overline{\mathrm{P}})=\hat{\pi}\left(\overline{\mathrm{P}^{\prime}}\right)$ but with $\check{\pi}\left(\overline{\mathrm{F}}_{1}\right) \neq \check{\pi}\left(\overline{\mathrm{F}}_{2}\right)$.

Let us consider the following open set $\mathrm{M}$ of the three-dimensional Minkowski spacetime (with the induced metric):

$$
M=\mathbb{L}^{3} \backslash\left(C_{1} \cup C_{2} \cup \cup_{n} l_{n}\right)
$$




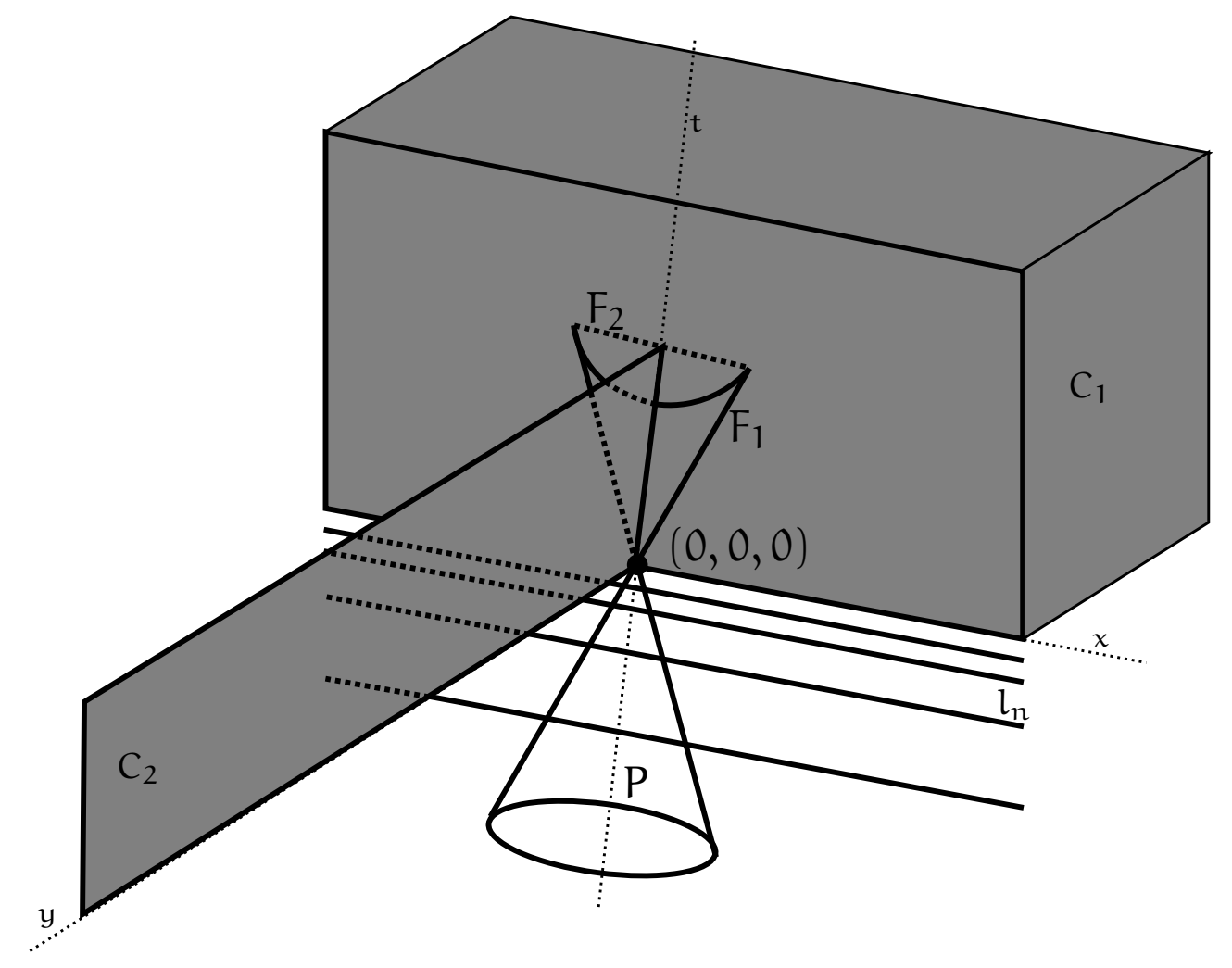

Figure 6. The space $M$ is an open set of the three-dimensional Minkowski spacetime with the sets $C_{1}, C_{2}$ and the sequence of lines $\left\{l_{n}\right\}$ removed. The point $(0,0,0)$ is represented on the c-boundary of $\mathrm{M}$ as two points $\left(\mathrm{P}, \mathrm{F}_{1}\right)$ and $\left(\mathrm{P}, \mathrm{F}_{2}\right)$. As happen in figure 5 , the lift of $\mathrm{P}$ to a fixed fibre of the universal cover of $M$ give us two terminal sets $\bar{P}$ and $\overline{P^{\prime}}$. In particular, and recalling again that $\uparrow \overline{\mathrm{P}}=\emptyset$, we deduce that the pairs $\left(\overline{\mathrm{P}}^{\prime}, \overline{\mathrm{F}}_{1}\right),\left(\overline{\mathrm{P}}^{\prime}, \overline{\mathrm{F}}_{2}\right)$ and $(\overline{\mathrm{P}}, \emptyset)$ belong to $\partial \mathrm{V}$, the c-boundary of the universal cover of $M$.

where $\mathrm{C}_{1}=\left\{(x, y, t) \in \mathbb{R}^{3}: \mathrm{y} \leqslant 0, \mathrm{t} \geqslant 0\right\}, \mathrm{C}_{2}=\left\{(0, \mathrm{y}, \mathrm{t}) \in \mathbb{R}^{3}: 0 \leqslant \mathrm{t} \leqslant 1\right\}$ and for $\mathrm{n} \in \mathbb{N}$, $l_{n}=\{(x, 0,-1 / n): x \in \mathbb{R}\}$ (see figure 6 ); and consider $V$ its universal covering.

The behaviour of the lifts/projections in this case works essentially as in previous example. In fact, if we project the figure into the plane $\mathrm{y}, \mathrm{z}$ we will obtain almost the same setting as in figure 5, with the first quadrant removed (and so, sharing the same properties). Hence, the set $\mathrm{P}$ is naturally lifted as two different terminal sets $\overline{\mathrm{P}}$ and $\overline{\mathrm{P}^{\prime}}$ living in the same fibre of $\mathrm{V}$. The main difference between this case and previous example is that, even if $\overline{\mathrm{P}}$ is still $\mathrm{S}$-related with the empty set, the set $\overline{\mathrm{P}^{\prime}}$ is $\mathrm{S}$-related with two sets, $\overline{\mathrm{F}}_{1}$ and $\overline{\mathrm{F}}_{2}$ corresponding to the lifts of $\mathrm{F}_{1}$ and $\mathrm{F}_{2}$. Therefore, the points $\left(\overline{\mathrm{P}}^{\prime}, \overline{\mathrm{F}}_{1}\right)$ and $\left(\overline{\mathrm{P}}^{\prime}, \overline{\mathrm{F}}_{2}\right)$ are both $\sim \mathrm{G}_{0}$-related with the pair $(\overline{\mathrm{P}}, \emptyset)$, while $\check{\pi}\left(\overline{\mathrm{F}}_{1}\right)=\mathrm{F}_{1} \neq \mathrm{F}_{2}=\check{\pi}\left(\overline{\mathrm{F}}_{2}\right)$.

This suggests two different possible definitions for the function $\alpha$, regarding the image of the point $(\overline{\mathrm{P}}, \emptyset)$. In fact, we can consider:

$$
\alpha_{1}((\overline{\mathrm{P}}, \emptyset))=\left(\overline{\mathrm{P}^{\prime}}, \overline{\mathrm{F}}_{1}\right) \quad \text { and } \quad \alpha_{2}((\overline{\mathrm{P}}, \emptyset))=\left(\overline{\mathrm{P}^{\prime}}, \overline{\mathrm{F}}_{2}\right)
$$

making $(\overline{\mathrm{P}}, \emptyset)$ projects to $\left(\mathrm{P}, \mathrm{F}_{1}\right)$ in the first case, or to $\left(\mathrm{P}, \mathrm{F}_{2}\right)$ on the second one. In 


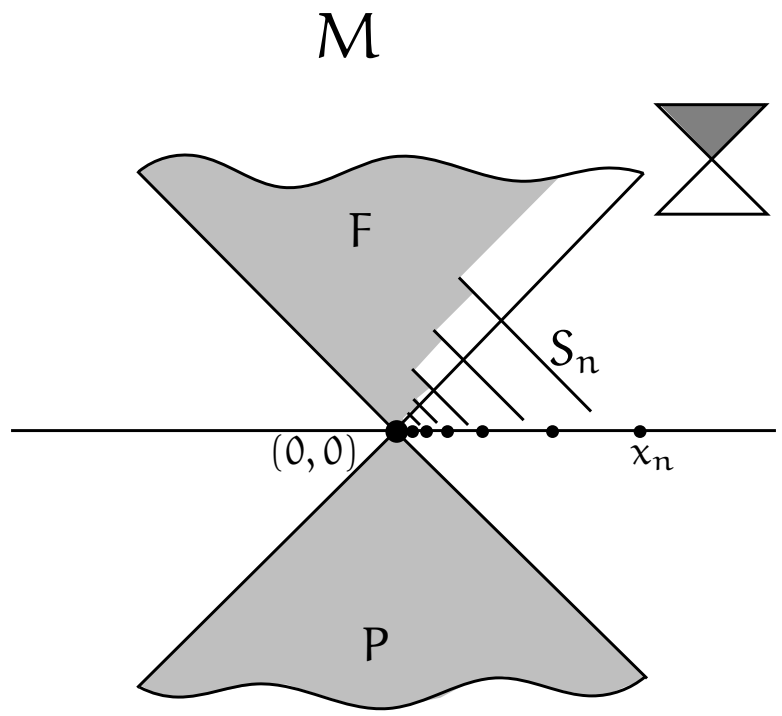

Figure 7. Let $M$ be $\mathbb{L}^{2}$ with the segments $S_{n}$ removed. On this example, the sets $P$ and $F$ are $S$-related and the sequence $\left\{x_{n}\right\}_{n}$ has $(P, F)$ on its limit. However, for $n$ big enough, the timelike curves from $x_{n}$ to points in $F$ should pass between $S_{n}$ and $S_{n+1}$. In particular, if $\left\{p_{n}\right\}_{n} \subset\{0\} \times M$ is a lift of $\left\{x_{\mathfrak{n}}\right\}_{\mathfrak{n}}$, and $\bar{F}$ is the corresponding lift of $F$ in $\{0\} \times M$, then $g \bar{F} \not \subset \operatorname{LI}\left(\left\{I^{+}\left(p_{\mathfrak{n}}\right)\right\}_{\mathfrak{n}}\right)$ for any $g \in G$.

both cases, the corresponding extended projections share the same properties: both restrict properly to $\bar{M}$, are surjective and non-continuous (as in previous example, it is possible to construct a sequence $\left\{\mathbf{q}_{\mathrm{n}}\right\}_{\mathrm{n}}$ converging to the pair $(\overline{\mathrm{P}}, \emptyset)$ whose projection $\left\{\mathrm{y}_{\mathrm{n}}\right\}$ does not converge to either $\left(\mathrm{P}, \mathrm{F}_{1}\right)$ nor $\left.\left(\mathrm{P}, \mathrm{F}_{2}\right)\right)$. So, as we pointed out in remark 4.5 , there are no actual differences between $\bar{\pi}_{\alpha_{1}}$ and $\bar{\pi}_{\alpha_{2}}$ regarding the satisfied properties.

Example 5.7. (Non open $\overline{1}$ ) Let us consider $\mathrm{M}$ a spacetime as in figure 7 and $\mathrm{V}$ the universal cover of $\mathrm{M}$. On $\mathrm{M}$, both sets $\mathrm{P}$ and $\mathrm{F}$ are $\mathrm{S}$-related and the sequence $\left\{x_{\mathrm{n}}\right\}_{\mathrm{n}}$ converges to the point $(\mathrm{P}, \mathrm{F})$. On $\mathrm{V}$, and thanks that we can take curves joining points from $\mathrm{P}$ to $\mathrm{F}$ without moving between any $\mathrm{S}_{\mathrm{n}}$ and $\mathrm{S}_{\mathrm{n}+1}$, we can obtain lifts $\overline{\mathrm{P}}$ and $\overline{\mathrm{F}}$ with $\overline{\mathrm{P}} \sim_{\mathrm{S}} \overline{\mathrm{F}}$ (we can assume that both sets live in the fibre $\{0\} \times M$ ).

However, no lift of the sequence $\left\{x_{n}\right\}_{n}$ converges to $(\overline{\mathrm{P}}, \overline{\mathrm{F}})$. In fact, let us take $\left\{\mathrm{p}_{\mathrm{n}}\right\}_{\mathrm{n}}$ a fixed lift of $\left\{x_{n}\right\}_{n}$ contained in $\{0\} \times M$. It is not difficult to observe that this lift is the only one satisfying that $\overline{\mathrm{P}} \in \hat{\mathrm{L}}_{\mathrm{V}}\left(\left\{\mathrm{I}^{-}\left(\mathrm{p}_{\mathrm{n}}\right)\right\}_{\mathfrak{n}}\right)$. Even so, it is not true that $\overline{\mathrm{F}} \in \check{\mathrm{L}}_{\mathrm{V}}\left(\left\{\mathrm{I}^{+}\left(\mathrm{p}_{\mathrm{n}}\right)\right\}_{\mathfrak{n}}\right)$, as any timelike curve joining a point $\mathrm{x}_{\mathrm{n}}$ with points on $\mathrm{F}$ should pass through two lines $S_{\mathfrak{n}}$, hence its lift moves between two different fibres. Therefore, the sequence $\left\{x_{\mathfrak{n}}\right\}_{\mathfrak{n}}$ has no natural convergent lift and the map $\bar{\jmath}$ is not open.

Finally, let us observe that $\hat{\jmath}$ and $\breve{\jmath}$ are continuous. This follows by reasoning as in example 5.2, recalling that the only cases where the continuity could fail is considering sequences $\left\{y_{n}\right\}_{n}$ converging to $(0,0)$.

Example 5.8. ( $\bar{V}$ with no lightlike boundary points while $\bar{M}$ has them) Let

$$
M=\left(\left\{(x, t) \in \mathbb{R}^{2}: 0<x<1,-1<t \leqslant x\right\} \cup([1,2) \times(-1,1))\right) \backslash \cup_{n} S_{n}
$$


M

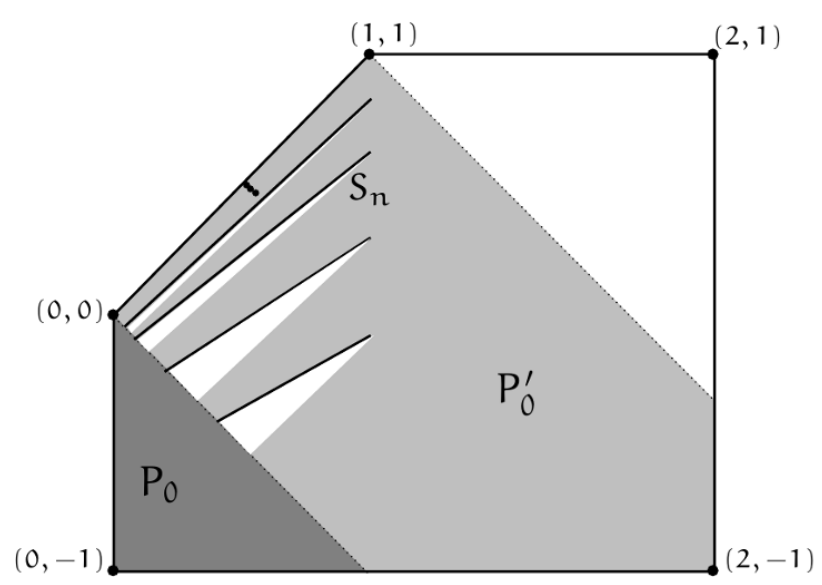

$\partial M$

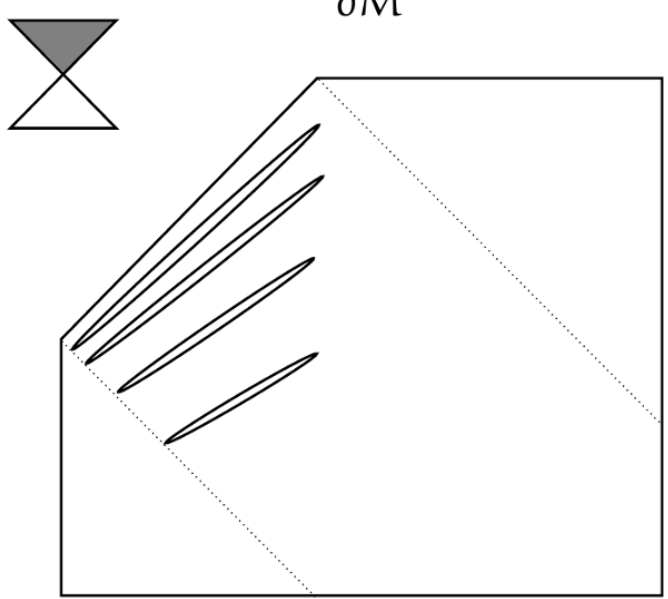

Figure 8. Let $M$ be an open set of $\mathbb{L}^{2}$ with the segments $S_{n}$ removed as in the left. Even if the segments are spacelike, the terminal set $P_{0}^{\prime}$ (the past of the boundary point $(1,1)$ ) contains $P_{0}$ (the past of $(0,0))$.

The c-boundary of $M$ is represented on the right of the figure. Observe that, in the c-boundary, each segment $S_{n}$ is represented by a thin ellipse. This is due the fact that any non-extremal point of the segment is reachable by a future and past inextensible timelike curve, but the corresponding terminal sets are not $S$-related. So, such points are represented in the c-boundary as two points of the form $(P, \emptyset)$ and $(\emptyset, F)$. Only on the extremal points the corresponding TIP and TIF are $S$-related, and so, they determine only one point in the c-boundary.

be a manifold as in figure 8 endowed with the induced Minkowski metric, where each $\mathrm{S}_{\mathrm{n}}$ is a spacelike segment obtained from a small variation of the lightlike segment joining $(1 / n,-1 / n)$ and $(1,1-2 / n)$. Due the fact that $(1 / n,-1 / n) \ll(1,1-2 /(n+1))$, such a variation can be taken in such a way that the past of the upper-right extreme of $\mathrm{S}_{\mathrm{n}+1}$ contains the down-left extreme of $\mathrm{S}_{\mathrm{n}}$. Let $\mathrm{V}$ be the universal cover of $\mathrm{M}$.

The c-boundary (and so, the c-completion) of $\mathrm{M}$ is represented on the right of figure 8 and it is formed almost entirely by spatial and timelike boundary points. However, the points $(0,0)$ and $(1,1)$ are represented on the boundary by pairs of the form $\left(\mathrm{P}_{0}, \emptyset\right)$ and $\left(\mathrm{P}_{0}^{\prime}, \emptyset\right)$ with $\mathrm{P}_{0} \subsetneq \mathrm{P}_{0}^{\prime},{ }^{5}$ hence $\overline{\mathrm{M}}$ has lightlike boundary points. Topologically the c-completion $\overline{\mathrm{M}}$ is Hausdorff, as it has the induced topology from $\mathbb{R}^{2}$.

Now, if we look into the lifts of boundary points from $\bar{M}$ to $\overline{\mathrm{V}}$, we observe that timelike and spatial boundary points are lifted to timelike and spatial boundary points respectively. However, there exist no lifts $\left(\overline{\mathrm{P}}_{0}, \emptyset\right)$ and $\left(\overline{\mathrm{P}}_{0}^{\prime}, \emptyset\right)$ of $\left(\mathrm{P}_{0}, \emptyset\right)$ and $\left(\mathrm{P}_{0}^{\prime}, \emptyset\right)$ respectively such that $\overline{\mathrm{P}}_{0} \subsetneq \overline{\mathrm{P}}_{0}$, as any timelike curve moving from a point close to $(1,1)$ to a point close to $(0,0)$ should move between two segments $S_{m}$ and $S_{m+1}$, and so, it will move between different fibres of $\mathrm{V}$ (recall again the behaviour of the universal covering described on example 5.1). Therefore, $\overline{\mathrm{V}}$ will have no lightlike boundary points. Finally, and due the fact that the topology around a point of $\overline{\mathrm{V}}$ coincides again with the induced topology from $\mathbb{R}^{2}$, we have that $\overline{\mathrm{V}}$ is also Hausdorff.

\footnotetext{
${ }^{5}$ Observe that $(1 / n,-1 / n) \ll(1,1-2 /(n+1))$, so it is possible to obtain timelike curves passing between $S_{n}$ and $S_{n+1}$.
} 


\section{A physical application: quotients on Robertson-Walker spacetimes}

As a final section of this paper, we will show how our results are applicable to concrete and physically relevant models of spacetimes. Our main aim will be to apply corollaries 4.24 and 4.26 where, in addition to the finite chronology, we need Hausdorffness on both $\hat{V}$ and $\check{V}$ and the non existence of lightlike boundary points on $\bar{M}$ (recall also corollary 4.25).

We will focus on the case of Robertson Walker models, even if our results are extensible to other more general ones (see remark 6.4). The c-completion of such a models is well known [17, section 4.2], but we include here the details for completeness. Observe that we are not going to follow the original approach proposed in [17], but the approach introduced in $[20$, section 3].

Let $\left(\Sigma, \mathfrak{g}_{\Sigma}\right)$ be a Riemannian manifold. Denote by $\mathrm{t}: \mathbb{R} \times \Sigma \rightarrow \mathbb{R}$ and $\pi_{\Sigma}: \mathbb{R} \times \Sigma \rightarrow \Sigma$ the corresponding projections; and consider a smooth positive function $\alpha: \mathbb{R} \rightarrow(0, \infty)$. A Robertson Walker model with base $\Sigma$ and warping function $\alpha$ is given then by the pair $(\mathrm{V}, \mathfrak{g})$, where

$$
\mathrm{V}=\mathbb{R} \times \Sigma, \quad \text { and } \quad \mathfrak{g}=-\mathrm{dt}^{2}+(\alpha \circ \mathrm{t}) \pi_{\Sigma}^{*}\left(\mathfrak{g}_{\Sigma}\right) .
$$

For simplicity, $\alpha \circ \mathrm{t}$ will be denoted just by $\alpha(\mathrm{t})$ and, whenever there is no confusion, we will omit the pullback $\pi_{\Sigma}^{*}$. The chronological relation on these models is characterized as (see [20, proposition 3.1]):

$$
\left(t_{0}, x_{0}\right) \ll\left(t_{1}, x_{1}\right) \Longleftrightarrow d\left(x_{0}, x_{1}\right)<\int_{t_{0}}^{t_{1}} \frac{1}{\sqrt{\alpha(s)}} d s
$$

where $\mathrm{d}$ denotes the distance on $\Sigma$ defined by $\mathfrak{g}_{\Sigma}$. Thanks to previous characterization, it follows that any future terminal set $\mathrm{P}$ is determined by the so-called Busemann functions. Such functions are defined in the following way: given a curve $c:[a, \Omega) \rightarrow \Sigma$ satisfying that $\mathfrak{g}_{\Sigma}(\dot{\boldsymbol{c}}, \dot{\mathfrak{c}})<1$, we define the associated Busemann function as:

$$
b_{c}(\cdot)=\lim _{t \rightarrow \Omega}\left(\int_{0}^{t} \frac{1}{\sqrt{\alpha(s)}} d s-d(\cdot, c(t))\right)
$$

Then, for any indecomposable past set $\mathrm{P}$, it follows that $\mathrm{P}=\mathrm{P}\left(\mathrm{b}_{\mathfrak{c}}\right)$ for some curve $\mathrm{c}$ with $\mathfrak{g}_{\Sigma}(\dot{\mathrm{c}}, \dot{\mathrm{c}})<1$, where

$$
\mathrm{P}\left(\mathrm{b}_{\mathrm{c}}\right)=\left\{(\mathrm{t}, \mathrm{x}) \in \mathrm{V}: \int_{0}^{\mathrm{t}} \frac{1}{\sqrt{\alpha(s)}} \mathrm{ds}<\mathrm{b}_{\mathrm{c}}(\mathrm{x})\right\}
$$

(see [20, Equation (3.3)]). If we have either $\Omega<\infty$; or $\Omega=\infty$ and $\int_{0}^{\infty} \frac{1}{\sqrt{\alpha(s)}} \mathrm{ds}<\infty$; it follows that $\mathrm{c}(\mathrm{t}) \rightarrow \chi^{*} \in \Sigma_{\mathrm{C}}$, where $\Sigma_{\mathrm{C}}$ denotes the Cauchy completion associated to $\left(\Sigma, g_{\Sigma}\right)$. Moreover, $b_{c}(\cdot)=d_{\left(\Omega, x^{*}\right)}(\cdot):=\int_{0}^{\Omega} \frac{1}{\sqrt{\alpha(s)}} \mathrm{d} s-\mathrm{d}\left(\cdot, x^{*}\right)$ (see [20, Equations (3.7) and (3.8)]. In this way, and under the assumption of previous integral condition, we have that the future causal completion has the following point set structure:

$$
\hat{V} \equiv \Sigma_{C} \times\{\mathbb{R} \cup\{\infty\}\}
$$


The study is completely analogous for the past orientation, where if we assume the integral condition $\int_{-\infty}^{0} \frac{1}{\sqrt{\alpha}(s)} d s<\infty$, the past causal completion is identified with:

$$
\check{V} \equiv \Sigma_{C} \times\{\mathbb{R} \cup\{-\infty\}\}
$$

Finally, for the (total) c-completion, we only need to observe that past and future indecomposable past sets are $S$-related if they are associated to the same pair $\left(\Omega, x^{*}\right) \in$ $\mathbb{R} \times \Sigma_{C}$ (see [20, Equation (3.14)] and the paragraph above). In conclusion, the following result follows:

Proposition 6.1. Let $(\mathrm{V}, \mathfrak{g})$ be a Robertson Walker model as in (6.1), and assume the following integral conditions

$$
\int_{0}^{\infty} \frac{1}{\sqrt{\alpha(s)}} \mathrm{d} s<\infty, \quad \int_{-\infty}^{0} \frac{1}{\sqrt{\alpha(s)}} \mathrm{d} s<\infty .
$$

Then, the c-completion, as point set, becomes

$$
\overline{\mathrm{V}} \equiv \Sigma_{\mathrm{C}} \times\{\{-\infty\} \cup \mathbb{R} \cup\{\infty\}\} .
$$

Chronologically, the c-boundary has two copies, one for the future and one for the past, of the Cauchy completion $\Sigma_{\mathrm{C}}$ formed by spatial boundary points; and timelike lines over each point of the Cauchy boundary of $\Sigma$. Topologically, and assuming that $\Sigma_{\mathrm{C}}$ is locally compact, the chronological topology on $\overline{\mathrm{V}}$ coincides with the product topology in $\Sigma_{C} \times\{\{-\infty\} \cup \mathbb{R} \cup\{\infty\}\}$. Morever, both $\hat{\mathrm{V}}$ and $\check{\mathrm{V}}$ are Hausdorff.

Proof. The pointset and causal structure can be deduced from previous comments (see also [17, theorem 4.2]). For the topological structure, we only need to recall that [31, proposition 5.24] is also applicable to this approach and, moreover, it is also true when $\Omega=\infty$ if the integral condition holds.

Therefore, when the integral conditions are satisfied and the associated Cauchy completion $\Sigma_{C}$ is locally compact, both $\hat{V}$ and $\check{V}$ are Hausdorff and $\bar{V}$ has no lightlike boundary points. Therefore, and as a consequence of corollary 4.26 :

Theorem 6.2. Let $(\mathrm{V}, \mathfrak{g})$ be a Robertson Walker model as in (6.1) and assume both, the integral conditions in (6.2) and that $\Sigma_{\mathrm{C}}$ is locally compact. Then, if $\pi: \mathrm{V} \rightarrow \mathrm{M}$ is a spacetime covering projection with associated group $\mathrm{G},(\mathrm{V}, \mathrm{G})$ is finitely chronological and the $\mathrm{G}$-orbits are closed for both $\hat{\mathrm{V}}$ and $\check{\mathrm{V}}$, then $\overline{\mathrm{V}} / \mathrm{G}$ and $\overline{\mathrm{M}}$ are both, chronologically isomorphic and homeomorphic.

Obviously, our results are applicable in other Robertson Walker models without the integral conditions (6.2). For instance, the Anti-de Sitter model also satisfy both, it has Hausdorff partial completions and has no lightlike boundary points (see [17, section 4.1]). Moreover, the only pairs in $\overline{\mathrm{V}}$ with an empty component are of the form $(\mathrm{V}, \emptyset)$ and $(\emptyset, \mathrm{V})$, corresponding to $i^{+}$and $i^{-}$, so it follows readily that $\bar{M}$ has no lightlike boundary points. Hence: 
Theorem 6.3. Let $(\mathrm{V}, \mathfrak{g})$ be the Anti-de Sitter model with a timelike line for the origin removed, that is, $\mathrm{V}=\mathbb{R} \times(0, \infty) \times \mathbb{S}^{2}$ and

$$
\mathfrak{g}=-\cosh ^{2}(r) d t^{2}+d r^{2}+\sinh ^{2}(r)\left(d \theta^{2}+\sin ^{2} \theta d \phi^{2}\right) .
$$

Assume that we have a spacetime covering projection $\pi: \mathrm{V} \rightarrow \mathrm{M}$ with associated group $\mathrm{G}$ in such a way that $(\mathrm{V}, \mathrm{G})$ is finitely chronological. Then, $\overline{\mathrm{V}} / \mathrm{G} \equiv \overline{\mathrm{M}}$.

Previous result can be used, for instance, to calculate the c-completion of the BTZ blackhole models [13] and the Hawking-Page reference model [14], which are obtained as suitable quotients of the 3-dimensional Anti-de Sitter model [15? , 16].

Remark 6.4. We would like to note finally that theorem 6.2 is generalizable to other, more general, models of spacetimes. For instance, a similar result follows for Lorentz manifolds $(\mathbb{R} \times \Sigma, \mathfrak{g})$ with

$$
\mathfrak{g}=-d t^{2}+\sqrt{\alpha \circ t} \pi_{\Sigma}^{*}(\omega) \otimes d t+\sqrt{\alpha \circ t} d t \otimes \pi_{\Sigma}^{*}(\omega)+(\alpha \circ t) \pi_{\Sigma}^{*}\left(\mathfrak{g}_{\Sigma}\right)
$$

where $\omega$ is a one-form of $\Sigma$. Observe that such metrics are generalizations of RobertsonWalker models to the standard stationary settings. In fact, the theory developed in [31] for the stationary case is enough to study their c-completion (see [20, section 3]).

The c-completion of the standard stationary case presents remarkable differences with respect to the Static one, mainly because its causality is no longer determined by a (regular) distance but by a (non-symmetric) generalized distance. That lack of symmetry is reflected on different structures for the future and past c-completions. For instance, future and past completions depends on different Cauchy completions (named by forward and backward Cauchy completions and denoted by $\Sigma_{\mathrm{C}}^{ \pm}$respectively, see [31, section 6$]$ ). However, an under some mild hypotheses (the local compactness of $\Sigma_{\mathrm{C}}^{ \pm}$and the well behaviour of the extended distance to such spaces, see [31, theorem 1.2]), it follows analogous versions of proposition 6.1 and theorem 6.2 for the model (6.3).

\section{Acknowledgments}

Both authors would like to express their gratitude to Profs. J.L. Flores, M. Sánchez and S. Harris for suggesting some improvements on the first version of this paper (including example 5.4). They would also like to thanks the anonymous referee for his/her constructive comments.

The authors are partially supported by the Spanish Grant MTM2013-47828-C2-2-P (MINECO and FEDER funds). L. Aké also acknowledges a grant funded by the Consejo Nacional de Ciencia y Tecnología (CONACyT), México. JH would like to thank the Department of Algebra, Geometry and Topology of the University of Málaga for their kind hospitality while part of the work on this paper was being carried out. 
Open Access. This article is distributed under the terms of the Creative Commons Attribution License (CC-BY 4.0), which permits any use, distribution and reproduction in any medium, provided the original author(s) and source are credited.

\section{References}

[1] J.M. Maldacena, The Large-N limit of superconformal field theories and supergravity, Int. J. Theor. Phys. 38 (1999) 1113 [hep-th/9711200] [INSPIRE].

[2] A. Garcia-Parrado and J.M.M. Senovilla, Causal structures and causal boundaries, Class. Quant. Grav. 22 (2005) R1 [gr-qc/0501069] [INSPIRE].

[3] S.G. Harris, Boundaries on spacetimes: causality, topology, and group actions, Geom. Dedicata 126 (2007) 255.

[4] M. Sánchez, Causal boundaries and holography on wave type spacetimes, Nonlinear Anal. 71 (2009) e1744.

[5] J.L. Flores, J. Herrera and M. Sanchez, On the final definition of the causal boundary and its relation with the conformal boundary, Adv. Theor. Math. Phys. 15 (2011) 991 [arXiv: 1001.3270] [INSPIRE].

[6] J.L. Flores, J. Herrera and M. Sánchez, Hausdorff separability of the boundaries for spacetimes and sequential spaces, J. Math. Phys. 57 (2016) 022503.

[7] P.T. Chrusciel, Conformal boundary extensions of Lorentzian manifolds, J. Diff. Geom. 84 (2010) 19 [gr-qc/0606101] [INSPIRE].

[8] D. Berenstein, J. Maldacena and H. Nastase, Strings in flat space and pp waves from $\mathcal{N}=4$ super Yang Mills, J. High Energy Phys. (2002) 30.

[9] D. Marolf and S.F. Ross, Plane waves: To infinity and beyond!, Class. Quant. Grav. 19 (2002) 6289 [hep-th/0208197] [INSPIRE].

[10] D. Marolf and S.F. Ross, A New recipe for causal completions, Class. Quant. Grav. 20 (2003) 4085 [gr-qc/0303025] [inSPIRE].

[11] J. L. Flores and M. Sánchez, The causal boundary of wave-type spacetimes, J. High Energy Phys. (2008) 036.

[12] G. Mess, Lorentz spacetimes of constant curvature, Geometriae Dedicata 126 (2007) 3.

[13] M. Bañados, C. Teitelboim and J. Zanelli, The Black hole in three-dimensional space-time, Phys. Rev. Lett. 69 (1992) 1849 [hep-th/9204099] [InSPIRE].

[14] S.W. Hawking and D.N. Page, Thermodynamics of black holes in anti-de Sitter space, Comm. Math. Phys. 87 (1982/83) 577.

[15] M. Bañados, M. Henneaux, C. Teitelboim and J. Zanelli, Geometry of the (2+1) black hole, Phys. Rev. D 48 (1993) 1506 [Erratum ibid. D 88 (2013) 069902] [gr-qc/9302012] [INSPIRE].

[16] E. Witten, Anti-de Sitter space, thermal phase transition and confinement in gauge theories, Int. J. Mod. Phys. A 16 (2001) 2747.

[17] V. Alana and J.L. Flores, The Causal boundary of product spacetimes, Gen. Rel. Grav. 39 (2007) 1697 [arXiv:0704.3148] [INSPIRE]. 
[18] J.R. Gott, III, Closed timelike curves produced by pairs of moving cosmic strings: Exact solutions, Phys. Rev. Lett. 66 (1991) 1126 [INSPIRE].

[19] S.G. Harris, Discrete group actions on space-times: Causality conditions and the causal boundary, Class. Quant. Grav. 21 (2004) 1209 [gr-qc/0310071] [InSPIRE].

[20] J. Flores, J. Herrera and M. Sánchez, Computability of the causal boundary by using isocausality, Classical Quant. Grav. 30 (2013) 075009.

[21] R. Geroch, E.H. Kronheimer and R. Penrose, Ideal points in space-time, Proc. Roy. Lond. Soc. A 327 (1972) 545.

[22] R. Budic and R.K. Sachs, Causal boundaries for general relativistic space times, J. Math. Phys. 15 (1974) 1302.

[23] S.G. Harris, Universality of the Future Chronological Boundary, J. Math. Phys. 39 (1998) 5427 [gr-qc/9704011] [INSPIRE].

[24] S.G. Harris, Topology of the future chronological boundary: Universality for space-like boundaries, Class. Quant. Grav. 17 (2000) 551 [gr-qc/9907062] [INSPIRE].

[25] I. Racz, Causal boundary of space-times, Phys. Rev. D 36 (1987) 1673 [InSPIRE].

[26] L.B. Szabados, Causal Boundary for Strongly Causal Space-time, Class. Quant. Grav. 5 (1988) 121 [INSPIRE].

[27] L.B. Szabados, Causal boundary for strongly causal spacetimes. II, Classical Quant. Grav. 6 (1989) 77

[28] R. Wald, General Relativity. University of Chicago Press, Chicago, U.S.A. (1984).

[29] J.L. Flores, The Causal Boundary of spacetimes revisited, Commun. Math. Phys. 276 (2007) 611 [gr-qc/0608063] [INSPIRE].

[30] E. Minguzzi and M. Sánchez, The causal hierarchy of spacetimes, in Recent developments in pseudo-Riemannian geometry, ESI Lect. Math. Phys. (2008) 299.

[31] J.L. Flores, J. Herrera and M. Sánchez, Gromov, Cauchy and causal boundaries for Riemannian, Finslerian and Lorentzian manifolds, Mem. Am. Math. Soc. 226 (2013) vi+76. 